\title{
Novel Chiral Ionic Liquid (CIL) Assisted Selectivity Enhancement to (L)-Proline Catalyzed Asymmetric Aldol Reactions
}

\author{
Long Zhang, Haibo Zhang,* Huadong Luo, Xiaohai Zhou* and Gongzhen Cheng \\ College of Chemistry, Wuhan University, 430072, P. R. China
}

\begin{abstract}
Melhoras significativas nos rendimentos químicos (até 88\%), estereosseletividades (>99:1) e excessos enantioméricos (até $98 \%$ ) foram observadas em reações aldólicas assimétricas catalisadas por (L)-prolina, quando líquidos iônicos quirais baseados em prolina (CILs) foram usados como aditivos. Diferentes proporções de DMSO/ $\mathrm{H}_{2} \mathrm{O}$ como solvente, e líquidos iônicos quirais (CILs) com cátions quirais com diferentes comprimentos de cadeia, foram investigados.
\end{abstract}

A significant improvement of the chemical yields (up to 88\%), stereoselectivity (>99:1) and enantiomeric excesses (up to 98\%) of (L)-proline catalyzed direct asymmetric aldol reaction was found when proline based chiral ionic liquids (CILs) were added as additives. Different ratios of DMSO/ $\mathrm{H}_{2} \mathrm{O}$ as solvent and chiral ionic liquids (CILs) with chiral cations of different chain length were investigated.

Keywords: aldol reaction, asymmetric synthesis, chiral ionic liquids, chiral additive

\section{Introduction}

Room temperature ionic liquids (RTILs) including chiral ionic liquids (CILs) have received growing attentions both from theoretical research and actual productions due to their tuneable features for various chemical tasks and their advantages as homogeneous support, reaction media, etc. ${ }^{1,2}$ Since the first example of chiral ionic liquid reported by Howarth et al. ${ }^{3}$ in 1997, a large number of CILs bearing chiral cations, anions or seldom both were reported by other groups. ${ }^{4,5}$ Previous studies have shown that in many asymmetric reactions the use of CILs as reaction media or catalyst can enhance the yield and/or selectivity significantly. ${ }^{6-8}$ As most of the asymmetric reactions were carried out using metal/chiral catalysts as chiral sources, which are relatively expensive or environmental unfriendly, searching for a catalyst which is easy to obtain and environmentally benign for the asymmetric reactions is of great significance. In our previous work, a series of (L)-proline based CILs were proved to have the potential to provide a strong chiral environment by ${ }^{19} \mathrm{~F}$ NMR study. ${ }^{9}$ In this article, a series of reactions were carried out to investigate the catalytic properties of these (L)-proline based CILs, and very high chiral selectivity was obtained.

*e-mail: haobozhang1980@gmail.com, zxh7954@hotmail.com
Aldol reaction has been a typical asymmetric reaction used in the search of efficient asymmetric catalyst, especially for environmental friendly and metal-free organic catalyst. ${ }^{10-13}$ Since CILs used as catalyst in aldol reaction by Luo et al. ${ }^{14}$ in 2007, many CILs were developed as catalysts for asymmetric reactions. However, in asymmetric reactions where CILs cannot be applied as catalysts, using CILs as the solvent to investigate the chiral inducing capabilities has rarely been reported. Here we chose the asymmetric aldol reactions as our initial focus to examine the applications of CILs in asymmetric reactions. (L)-Proline and its structural analogs were found to be good catalyst for aldol reaction, ${ }^{15-19}$ and (L)-proline combined with ILs were also proved to be an efficient catalytic system for asymmetric aldol reactions..$^{20-23}$ However, a systematic study about the relationship between structure and properties of ionic liquids is of great interest to many researchers.

\section{Results and Discussion}

In this work, (L)-proline based CILs (Scheme 1) are used as chiral additives for (L)-proline-catalyzed asymmetric aldol reaction between aldehyde and acetone, and the results are listed in Table 1. CILs alone can not promote this reaction (Table 1, entry 1). Upon addition 


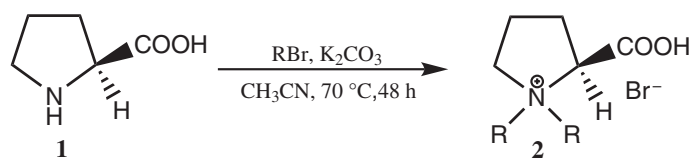

2a: $\mathrm{R}=\mathrm{CH}_{2} \mathrm{CH}_{3} \quad$ 2d: $\mathrm{R}=\left(\mathrm{CH}_{2}\right)_{7} \mathrm{CH}_{3}$ 2b: $\mathrm{R}=\left(\mathrm{CH}_{2}\right)_{3} \mathrm{CH}_{3} \quad$ 2e: $\mathrm{R}=\left(\mathrm{CH}_{2}\right)_{9} \mathrm{CH}_{3}$ 2c: $\mathrm{R}=\left(\mathrm{CH}_{2}\right)_{5} \mathrm{CH}_{3} \quad$ 2f: $\mathrm{R}=\left(\mathrm{CH}_{2}\right)_{11} \mathrm{CH}_{3}$

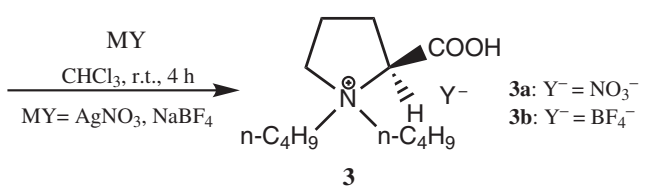

Scheme 1. Synthesis of CILs.

of (L)-proline even with a slight amount the reactions proceeded at room temperature and gave the aldol product.

The yields were obviously increased with increasing amount of (L)-proline (Table 1, entries 2 to 4). When (L)-proline (30 $\mathrm{mol} \%)$ and $\mathbf{2 b}(7.5 \mathrm{~mol} \%)$ in an acetone mixture of the reactants were used, the reaction proceeded with a superior yield (up to 97\%) and a moderate $e e$ value $(71 \%)$. An obvious decrease of the yield but a slight increase of the $e e$ values can be observed with decreasing amount of (L)-proline. This result is in accordance with that of Jacobsen's catalytic mechanism. ${ }^{25}$ It can be concluded that the amount of catalyst have a direct relationship with the yield but a slight influence on the reaction selectivity. For the CILs, when the amount of CILs varied from $1 \mathrm{~mol} \%$ to $7.5 \mathrm{~mol} \%$, it was found almost no change in the reaction yield, while the by product due to dehydration increased slightly with the increase of CILs. The ee values definitely increased with the addition of CILs $\mathbf{2} \mathbf{b}$, although very slightly. Using different aromatic aldehyde in the (L)proline catalyzed aldol reaction, adding $\mathbf{2} \mathbf{b}$ as additive and as well good yield and selectivity, the enantiomeric excesses can have up to $24 \%$ enhancement.

In summary of the results above, the best reaction ratio of catalyst and additives were found to be $30 \mathrm{~mol} \%$ (L)-proline as catalyst and $7.5 \mathrm{~mol} \%$ CILs as additives. Based on this founding, all of the following reactions were carried out on these conditions. The recycle ability of the catalyst and CILs were also studied (Table 1, entry $4 \mathrm{f}$-h). The reaction was taken $24 \mathrm{~h}$, the solvent was evaporated, and resides were extracted with $\mathrm{Et}_{2} \mathrm{O}$ three times. The combined solution was left for analysis, and resides were dried under vacuum for $3 \mathrm{~h}$ for the next cycle. The catalytic activity of the catalyst and CILs can be kept after 3 cycles, with only a slight decrease in $e e$ values. To investigate the generality of the CILs as additives, a series of aromatic aldehyde acceptors were subjected to the same reaction conditions. From the results above, an obvious ee enhancement change can be found when $-\mathrm{NO}_{2}$ was in different positions (entries 4 and 5, entries 9 and 10, entries 14 and 15). In details, the $-\mathrm{NO}_{2}$ in $-o$ and $-p$ position showed better result than that in the $-\mathrm{m}$ position. This is mainly due to the positive effect in nucleophilic addition by $-\mathrm{NO}_{2}$ in $-o$ and $-p$ position. Furthermore, other aromatic aldehydes with different substituents at $-p$ positions (entries 16 and 17, entries 18 and 19 , entries 20 and 21, entries 27 and 28, entries 29 and 30) were also investigated to validate the results, it can be seen that all of them react well in this catalytic system and almost no significant change in selectivity which reveals that substituents have less effect in the chemical selectivity. As for the CILs, their solubility can be adjusted by altering the alkyl chain. In details, $\mathbf{2 a}, \mathbf{2 b}$ and $\mathbf{2 c}$ have good solubility in water while the others with longer chain lengths are better miserable with low polarity solvent. The other two kinds of CILs with different (TG) counter ions also have their own unique proprieties. Thermogravimetric measurement reveals that these CILs have $\mathrm{T}_{\mathrm{d}}$ (thermal degradation temperature) in the order of $\mathrm{BF}_{4}^{-}>\mathrm{Br}^{-}>\mathrm{NO}_{3}^{-}$. Although their widely different proprieties were shown by different chain lengths and different counter ions, they showed no significant change both in chemical yields and selectivity. The mechanism is still under investigation. As temperature is an important factor in most of the asymmetric synthesis, lower the temperature (Table 1, entries 6 and 13) in our system showed only a small change in $e e$ values (2\% and 5\%, respectively).

In addition, the aldol reaction between cyclohexanone and 4-nitrobenzaldehyde in the presence of (L)-proline and 2 in DMSO/ $\mathrm{H}_{2} \mathrm{O}$ solvent was also investigated (Table 2). Similarly, the reactions were carried out using (L)-proline as catalyst with or without CILs. It was very interesting to find that when $10 \mathrm{~mol} \%$ of CILs was added, the yields, stereo-selectivity and enantiomeric excesses were enhanced significantly. The solvent, which is very important in organic reactions, was optimized by a series of comparison experiments use different ratio of $\mathrm{DMSO} / \mathrm{H}_{2} \mathrm{O}$ (Table 2, entries 1 to 4 ). It was found that the best results were<smiles>CC(=O)/C=C/c1ccc([N+](=O)[O-])cc1</smiles>

Scheme 2. (L)-proline-catalyzed aldol reaction of aromatic aldehyde with acetone. 
Table 1. (L)-Proline-catalyzed aldol reaction of aromatic aldehyde with acetone

\begin{tabular}{|c|c|c|c|c|c|c|c|}
\hline entry & 4-(R) & $\begin{array}{l}\text { (L)-Proline / } \\
\text { molar ratio }\end{array}$ & Product & $\begin{array}{c}\text { CILs / } \\
\text { molar ratio }\end{array}$ & Conv. / (\%) & Selec. / $(\%)^{\mathrm{b}}$ & $e e /(\%)^{c}$ \\
\hline 1 & $4-\mathrm{NO}_{2} \mathrm{C}_{6} \mathrm{H}_{4}$ & 0 & $5 a$ & $7.5 \% \mathbf{2 b}$ & - & - & - \\
\hline 2 & $4-\mathrm{NO}_{2} \mathrm{C}_{6} \mathrm{H}_{4}$ & $5 \%$ & $5 a$ & $7.5 \% \mathbf{2 b}$ & 26 & 95 & 73 \\
\hline 3 & $4-\mathrm{NO}_{2} \mathrm{C}_{6} \mathrm{H}_{4}$ & $10 \%$ & $5 a$ & $7.5 \% \mathbf{2 b}$ & 64 & 99 & 75 \\
\hline \multirow[t]{4}{*}{4} & $4-\mathrm{NO}_{2} \mathrm{C}_{6} \mathrm{H}_{4}$ & $30 \%$ & $5 a$ & $7.5 \% \mathbf{2 b}$ & 96 & $>99$ & 74 \\
\hline & & & & & $-^{\mathrm{i}}$ & $-^{\mathrm{i}}$ & $71^{\mathrm{f}}$ \\
\hline & & & & & $-^{\mathrm{i}}$ & $-^{\mathrm{i}}$ & $69^{g}$ \\
\hline & & & & & $-^{\mathrm{i}}$ & $-^{\mathrm{i}}$ & $69^{\mathrm{h}}$ \\
\hline 5 & $4-\mathrm{NO}_{2} \mathrm{C}_{6} \mathrm{H}_{4}$ & $30 \%$ & $5 a$ & 0 & $97^{\mathrm{d}}$ & $>99^{\mathrm{d}}$ & $66^{\mathrm{d}}$ \\
\hline 6 & $4-\mathrm{NO}_{2} \mathrm{C}_{6} \mathrm{H}_{4}$ & $30 \%$ & $5 a$ & $7.5 \% \mathbf{2 b}$ & $97^{\mathrm{e}}$ & $>99^{\mathrm{e}}$ & $76^{\mathrm{e}}$ \\
\hline 7 & $4-\mathrm{NO}_{2} \mathrm{C}_{6} \mathrm{H}_{4}$ & $30 \%$ & $5 a$ & $1 \% \mathbf{2 b}$ & 96 & 98 & 69 \\
\hline 8 & $4-\mathrm{NO}_{2} \mathrm{C}_{6} \mathrm{H}_{4}$ & $30 \%$ & $5 a$ & $5 \% \mathbf{2 b}$ & 97 & 96 & 71 \\
\hline 9 & $2-\mathrm{NO}_{2} \mathrm{C}_{6} \mathrm{H}_{4}$ & $30 \%$ & $5 b$ & $7.5 \% \mathbf{2 b}$ & 98 & $>99$ & 79 \\
\hline 10 & $2-\mathrm{NO}_{2} \mathrm{C}_{6} \mathrm{H}_{4}$ & $30 \%$ & $5 b$ & 0 & $98^{\mathrm{d}}$ & $>99^{d}$ & $72^{\mathrm{d}}$ \\
\hline 11 & $2-\mathrm{NO}_{2} \mathrm{C}_{6} \mathrm{H}_{4}$ & $30 \%$ & $5 b$ & $7.5 \% \mathbf{3 a}$ & 97 & $>99$ & 81 \\
\hline 12 & $2-\mathrm{NO}_{2} \mathrm{C}_{6} \mathrm{H}_{4}$ & $30 \%$ & $5 b$ & $7.5 \% \mathbf{3 b}$ & 97 & $>99$ & 81 \\
\hline 13 & $2-\mathrm{NO}_{2} \mathrm{C}_{6} \mathrm{H}_{4}$ & $30 \%$ & $5 b$ & $7.5 \% \mathbf{2 b}$ & $53^{\mathrm{e}}$ & $>99^{\mathrm{e}}$ & $84^{\mathrm{e}}$ \\
\hline 14 & $3-\mathrm{NO}_{2} \mathrm{C}_{6} \mathrm{H}_{4}$ & $30 \%$ & $5 c$ & $7.5 \% \mathbf{2 b}$ & 93 & 98 & 72 \\
\hline 15 & $3-\mathrm{NO}_{2} \mathrm{C}_{6} \mathrm{H}_{4}$ & $30 \%$ & $5 c$ & 0 & $91^{\mathrm{d}}$ & $>99^{\mathrm{d}}$ & $68^{\mathrm{d}}$ \\
\hline 16 & $\mathrm{C}_{6} \mathrm{H}_{5}$ & $30 \%$ & $5 d$ & $7.5 \% \mathbf{2 b}$ & 85 & 72 & 65 \\
\hline 17 & $\mathrm{C}_{6} \mathrm{H}_{5}$ & $30 \%$ & $5 d$ & 0 & 80 & 61 & $64^{\mathrm{d}}$ \\
\hline 18 & 4- $\mathrm{CH}_{3} \mathrm{C}_{6} \mathrm{H}_{5}$ & $30 \%$ & $5 e$ & $7.5 \% \mathbf{2 b}$ & 84 & 69 & 70 \\
\hline 19 & $4-\mathrm{CH}_{3} \mathrm{C}_{6} \mathrm{H}_{5}$ & $30 \%$ & $5 e$ & 0 & $65^{[\mathrm{d}]}$ & $82^{\mathrm{d}}$ & $64^{\mathrm{d}}$ \\
\hline 20 & $4-\mathrm{FC}_{6} \mathrm{H}_{4}$ & $30 \%$ & $5 f$ & $7.5 \% \mathbf{2 b}$ & 98 & $>99$ & 70 \\
\hline 21 & $4-\mathrm{FC}_{6} \mathrm{H}_{4}$ & $30 \%$ & $5 f$ & 0 & $98^{[\mathrm{d}]}$ & $>99^{d}$ & $67^{\mathrm{d}}$ \\
\hline 22 & 4- $\mathrm{FC}_{6} \mathrm{H}_{4}$ & $30 \%$ & $5 f$ & $7.5 \% \mathbf{2 a}$ & 97 & $>99$ & 70 \\
\hline 23 & $4-\mathrm{FC}_{6} \mathrm{H}_{4}$ & $30 \%$ & $5 f$ & $7.5 \% \mathbf{2 c}$ & 98 & $>99$ & 71 \\
\hline 24 & 4- $\mathrm{FC}_{6} \mathrm{H}_{4}$ & $30 \%$ & $5 f$ & $7.5 \% \mathbf{2 d}$ & 96 & $>99$ & 69 \\
\hline 25 & 4- $\mathrm{FC}_{6} \mathrm{H}_{4}$ & $30 \%$ & $5 f$ & $7.5 \% \mathbf{2 e}$ & 98 & $>99$ & 70 \\
\hline 26 & $4-\mathrm{FC}_{6} \mathrm{H}_{4}$ & $30 \%$ & $5 f$ & $7.5 \% \mathbf{2 f}$ & 97 & $>99$ & 69 \\
\hline 27 & $4-\mathrm{ClC}_{6} \mathrm{H}_{4}$ & $30 \%$ & $5 g$ & $7.5 \% \mathbf{2 b}$ & 90 & 88 & 71 \\
\hline 28 & 4- $\mathrm{ClC}_{6} \mathrm{H}_{4}$ & $30 \%$ & $5 g$ & 0 & $85^{\mathrm{d}}$ & $88^{\mathrm{d}}$ & $68^{\mathrm{d}}$ \\
\hline 29 & 4- $\mathrm{BrC}_{6} \mathrm{H}_{4}$ & $30 \%$ & $5 \mathrm{~h}$ & $7.5 \% \mathbf{2 b}$ & 92 & 89 & 70 \\
\hline 30 & 4- $\mathrm{BrC}_{6} \mathrm{H}_{4}$ & $30 \%$ & $5 \mathbf{h}$ & 0 & $90^{\mathrm{d}}$ & $86^{\mathrm{d}}$ & $67^{\mathrm{d}}$ \\
\hline
\end{tabular}

${ }^{\mathrm{a}}$ Based on the aldehyde recovery after column chromatography. ${ }^{\mathrm{b}}$ Determined by ${ }^{1} \mathrm{H}$ NMR. ${ }^{\mathrm{c}}$ Determined by HPLC. ${ }^{\mathrm{d}}$ Comparison results with (L)-proline as catalyst. ${ }^{\mathrm{e}}$ The reaction temperature is $-15^{\circ} \mathrm{C}$. ${ }^{\mathrm{fh}} \mathrm{Second}$, third, forth reuse of the CILs and catalysts, respectively. ${ }^{\mathrm{i}}$ Not detected.<smiles>O=Cc1ccc([N+](=O)[O-])cc1</smiles>

Scheme 3. CILs as additives for direct aldol reactions between 4-nitrobenzaldehyde and cyclohexanone. 
Table 2. CILs as additives for direct aldol reactions between 4-nitrobenzaldehyde and cyclohexanone

\begin{tabular}{|c|c|c|c|c|c|c|c|c|}
\hline \multirow{2}{*}{ entry } & \multirow{2}{*}{$\begin{array}{c}\text { Solvent } \\
{\left[\mathrm{DMSO}: \mathrm{H}_{2} \mathrm{O}\right]^{\mathrm{a}}}\end{array}$} & \multicolumn{3}{|c|}{ No additives } & \multicolumn{4}{|c|}{ Assisted by CILs } \\
\hline & & Yield / $(\%)^{\mathrm{b}}$ & $\mathrm{dr}\left[\right.$ syn:anti] ${ }^{\mathrm{c}}$ & $e e /(\%)^{\mathrm{d}}$ & CILs & Yield / $(\%)^{\mathrm{b}}$ & $\mathrm{dr}\left[\right.$ syn:anti] ${ }^{\mathrm{c}}$ & $e e /(\%)^{\mathrm{d}}$ \\
\hline 1 & $95: 5$ & 20 & $95: 5$ & 86 & $2 \mathrm{~b}$ & 60 & $99: 1$ & 93 \\
\hline 2 & $90: 10$ & 35 & $94: 6$ & 92 & $2 \mathbf{b}$ & 85 & $>99: 1$ & 97 \\
\hline 3 & $80: 20$ & 80 & $93: 7$ & 77 & $2 b$ & 90 & $>99: 1$ & 98 \\
\hline 4 & $70: 30$ & 60 & $92: 8$ & 73 & $2 b$ & 55 & $98: 2$ & 97 \\
\hline 5 & $80: 20$ & & & & $2 \mathbf{a}$ & 85 & $97: 3$ & 98 \\
\hline 6 & $80: 20$ & & & & $2 \mathrm{c}$ & 87 & $>99: 1$ & 97 \\
\hline 7 & $80: 20$ & & & & $2 d$ & 85 & $98: 2$ & 97 \\
\hline 8 & $80: 20$ & & & & $2 e$ & 88 & $>99: 1$ & 98 \\
\hline 9 & $80: 20$ & & & & $2 f$ & 86 & $>99: 1$ & 98 \\
\hline
\end{tabular}

${ }^{\mathrm{a}}$ Volume ratio of DMSO and $\mathrm{H}_{2} \mathrm{O}$. ${ }^{\mathrm{b}}$ Based on the aldehyde recovery after column chromatography. ${ }^{\mathrm{c}}$ Determined by ${ }^{1} \mathrm{H}$ NMR. ${ }^{\mathrm{d}}$ Determined by HPLC.

obtained when the DMSO: $\mathrm{H}_{2} \mathrm{O}=4: 1$ (Table 2, entry 3). The other CILs were also used as additives in this catalytic system (Table 2, entries 5 to 9). All of them showed superior co-catalytic effect in aldol reactions.

\section{Conclusions}

In summary, a novel asymmetric catalytic system with $\mathrm{N}$-substituted pyrrolidine-ionic liquids as chiral additives was developed and their potentials of providing chiral environment by direct asymmetric aldol reaction were demonstrated. This catalytic system can lead to high yields (up to 88\%), good stereoselectivities (>99:1) and superior enantiomeric excesses (up to $98 \%$ ) for syn-selective aldol reactions. This is the first use of chiral environment as chiral source to lead asymmetric reaction to our knowledge. Further studies on this kind of CILs in other asymmetric reactions are underway in our laboratory.

\section{Experimental}

\section{General procedure for chiral ionic liquid (CILs) synthesis}

(L)-Proline $(0.1 \mathrm{~mol})$ and 1-bromoalkane $(0.3 \mathrm{~mol})$ were mixed in a $100 \mathrm{~mL}$ round bottom flask with acetonitrile as solvent at $70{ }^{\circ} \mathrm{C}$ for 2 days in an inert atmosphere. After filtering, the solvent was removed by distillation and the raw products were washed with ether for several times. After purified by column chromatography $\left(\mathrm{CHCl}_{3}: \mathrm{EtOH}=\right.$ $20: 1$ ), the product was dried under vacuum for $5 \mathrm{~h}$ to afford the chiral ionic liquids (CILs) 2a-2f.

2b $(0.02 \mathrm{~mol})$ and silver nitrate (or tetrafluoroborate ammonium) $(0.02 \mathrm{~mol}$ ) were stirred at room temperature for $4 \mathrm{~h}$ with chloroform as solvent. After filtering, the solvent was distilled off and the desired raw chiral ionic liquids
(CILs) 3a and $\mathbf{3 b}$ were obtained. After purified by column chromatography $\left(\mathrm{CHCl}_{3}: \mathrm{EtOH}=20: 1\right)$, the product was dried under vacuum for $5 \mathrm{~h}$ to afford the final chiral ionic liquids (CILs) 3a and $\mathbf{3 b}$.

\section{Characterization of CILs}

\section{CIL $2 a$}

${ }^{1} \mathrm{H}$ NMR (300 MHz, $\left.\mathrm{D}_{2} \mathrm{O}\right) \delta 4.19$ (dq, 3H, $J 21.3,7.3$ ), 3.63 (ddd, $1 \mathrm{H}, J 11.6,7.7,4.3$ ), 3.28 (dq, $1 \mathrm{H}, J$ 14.6, 7.3), $3.17-2.97$ (m, 2H), 2.29 (ddd, 2H, J 92.6, 11.4, 6.5), 2.05$2.00(\mathrm{~m}, 1 \mathrm{H}), 1.87$ (ddd, $1 \mathrm{H}, J 19.6,14.4,12.9), 1.15$ (dd, $6 \mathrm{H}, J 13.2,7.1) .{ }^{13} \mathrm{C}$ NMR $\left(75 \mathrm{MHz}, \mathrm{D}_{2} \mathrm{O}\right) \delta 12.2,15.1$, $24.2,30.0,52.7,56.4,65.9,68.3,171.4)$.

\section{CIL $2 b$}

${ }^{1} \mathrm{H}$ NMR (300 MHz, $\left.\mathrm{D}_{2} \mathrm{O}\right) \delta 4.42-4.22(1 \mathrm{H}, \mathrm{m}), 4.22-$ $4.03(2 \mathrm{H}, \mathrm{m}), 3.80-3.57(\mathrm{~m}, 1 \mathrm{H}), 3.33-2.96(\mathrm{~m}, 3 \mathrm{H}), 2.65-$ $2.21(\mathrm{~m}, 1 \mathrm{H}), 2.15-1.76(\mathrm{~m}, 3 \mathrm{H}), 1.69-1.39(\mathrm{~m}, 4 \mathrm{H}), 1.24$ (dtd, 4H, J 14.8, 7.4, 3.1), 0.76 (td, 5H, J 7.3, 2.3). ${ }^{13} \mathrm{C}$ NMR (75 MHz, $\left.\mathrm{D}_{2} \mathrm{O}\right) \delta 15.7,15.8,21.3,22.0,25.2,30.0,30.9$, $31.0,32.6,58.1,58.5,69.7,70.4,172.3$.

\section{CIL $3 a$}

${ }^{1} \mathrm{H}$ NMR (300 MHz, $\left.\mathrm{CDCl}_{3}, \mathrm{TMS}\right) \delta 0.78-0.92(\mathrm{~m}, 6 \mathrm{H}$, $\left.\mathrm{CH}_{3}\right), 1.22-1.38\left(\mathrm{~m}, 4 \mathrm{H}, \mathrm{CH}_{2}\right), 1.48-1.78\left(\mathrm{~m}, 4 \mathrm{H}, \mathrm{CH}_{2}\right)$, 2.02-2.26 (m, 3H, $\left.\mathrm{CH}_{2}\right), 2.42-2.56\left(\mathrm{~m}, 1 \mathrm{H}, \mathrm{CH}_{2}\right), 3.14-3.46$ $\left(\mathrm{m}, 3 \mathrm{H}, \mathrm{NCH}_{2}\right), 3.84\left(\mathrm{~s}, 1 \mathrm{H}, \mathrm{NCH}_{2}\right), 4.14(\mathrm{t}, 2 \mathrm{H}, J 6 \mathrm{~Hz}$, $\left.\mathrm{NCH}_{2}\right), 4.29$ (t, $\left.1 \mathrm{H}, J 8.4 \mathrm{~Hz}, \mathrm{CH}\right) ;{ }^{13} \mathrm{C}$ NMR $(75 \mathrm{MHz}$, $\left.\mathrm{CDCl}_{3}, \mathrm{TMS}\right) \delta 13.7,13.8,19.2,20.2,22.5,28.2,30.5$, $30.5,55.3,56.4,66.9,67.6,167.5$.

\section{CIL $3 b$}

${ }^{1} \mathrm{H}$ NMR $\left(300 \mathrm{MHz}, \mathrm{CDCl}_{3}, \mathrm{TMS}\right) \delta 0.68-0.94(\mathrm{~m}, 6 \mathrm{H}$, $\left.\mathrm{CH}_{3}\right), 1.14-1.40\left(\mathrm{~m}, 4 \mathrm{H}, \mathrm{CH}_{2}\right), 1.42-1.80\left(\mathrm{~m}, 4 \mathrm{H}, \mathrm{CH}_{2}\right)$, 
1.97-2.24 (m, 3H, $\left.\mathrm{CH}_{2}\right), 2.36-2.52\left(\mathrm{~m}, 1 \mathrm{H}, \mathrm{CH}_{2}\right), 3.06-$ $3.40\left(\mathrm{~m}, 3 \mathrm{H}, \mathrm{NCH}_{2}\right), 3.79$ (d, $\left.1 \mathrm{H}, J 4.5 \mathrm{~Hz}, \mathrm{NCH}_{2}\right), 4.12$ (t, $2 \mathrm{H}, J 6.6 \mathrm{~Hz}, \mathrm{NCH}_{2}$ ), 4.20-4.32 (m, $\left.1 \mathrm{H}, \mathrm{CH}\right) ;{ }^{13} \mathrm{C} \mathrm{NMR}$ (75 MHz, $\left.\mathrm{CDCl}_{3}, \mathrm{TMS}\right) \delta 13.5,13.7,19.1,19.7,22.9,27.9$, 28.7, 30.4, 56.1, 56.4, 67.3, 67.4, 168.8 .

\section{CIL $2 c$}

${ }^{1} \mathrm{H}$ NMR $\left(300 \mathrm{MHz}, \mathrm{CDCl}_{3}, \mathrm{TMS}\right) \delta 0.68-0.84(\mathrm{~m}, 6 \mathrm{H}$, $\left.\mathrm{CH}_{3}\right), 1.08-1.36\left(\mathrm{~m}, 12 \mathrm{H}, \mathrm{CH}_{2}\right), 1.44-1.88\left(\mathrm{~m}, 4 \mathrm{H}, \mathrm{CH}_{2}\right)$, 2.02-2.21 (m, 3H, $\left.\mathrm{CH}_{2}\right), 2.42-2.62\left(\mathrm{~m}, 1 \mathrm{H}, \mathrm{CH}_{2}\right), 3.13-3.40$ $\left(\mathrm{m}, 3 \mathrm{H}, \mathrm{NCH}_{2}\right), 3.71\left(\mathrm{~s}, 1 \mathrm{H}, \mathrm{NCH}_{2}\right), 4.10(\mathrm{t}, 2 \mathrm{H}, J 6.6 \mathrm{~Hz}$, $\mathrm{NCH}_{2}$ ), 4.26-4.38 (m, 1H, CH); ${ }^{13} \mathrm{C} \mathrm{NMR}\left(75 \mathrm{MHz}, \mathrm{CDCl}_{3}\right.$, TMS) $\delta$ 14.0, 14.0, 22.1, 22.5, 22.6, 25.6, 25.9, 26.7, 28.5, 28.6, 31.3, 31.5, 53.7, 53.7, 64.9, 67.0, 168.1 .

\section{CIL 2d}

${ }^{1} \mathrm{H}$ NMR (300 MHz, $\left.\mathrm{CDCl}_{3}, \mathrm{TMS}\right) \delta 0.72-0.88(\mathrm{~m}$, $\left.6 \mathrm{H}, \mathrm{CH}_{3}\right), 1.05-1.38\left(\mathrm{~m}, 20 \mathrm{H}, \mathrm{CH}_{2}\right), 1.52-1.94(\mathrm{~m}, 4 \mathrm{H}$, $\left.\mathrm{CH}_{2}\right), 2.15\left(\mathrm{~d}, 3 \mathrm{H}, \mathrm{J} 3.9 \mathrm{~Hz}, \mathrm{CH}_{2}\right), 2.47-2.63\left(\mathrm{~m}, 1 \mathrm{H}, \mathrm{CH}_{2}\right)$, 3.13-3.50 (m, 3H, $\left.\mathrm{NCH}_{2}\right), 3.52-3.74\left(\mathrm{~m}, 1 \mathrm{H}, \mathrm{NCH}_{2}\right), 4.14$ (t, $\left.2 \mathrm{H}, J 6.9 \mathrm{~Hz}, \mathrm{NCH}_{2}\right), 4.23-4.38(\mathrm{~m}, 1 \mathrm{H}, \mathrm{CH}) ;{ }^{13} \mathrm{C} \mathrm{NMR}$ (75 MHz, $\left.\mathrm{CDCl}_{3}, \mathrm{TMS}\right) \delta 14.2,14.2,22.1,22.7,22.7,25.9$, $25.9,27.0,28.5,28.5,29.3,29.3,29.3,29.3,31.8,31.9$, $53.9,53.9,66.9,66.9,168.1$.

\section{CIL $2 e$}

${ }^{1} \mathrm{H}$ NMR $\left(300 \mathrm{MHz}, \mathrm{CDCl}_{3}, \mathrm{TMS}\right) \delta$ 0.61-0.74 (m, $\left.6 \mathrm{H}, \mathrm{CH}_{3}\right), 0.96-1.22\left(\mathrm{~m}, 28 \mathrm{H}, \mathrm{CH}_{2}\right), 1.40-1.80(\mathrm{~m}, 4 \mathrm{H}$, $\left.\mathrm{CH}_{2}\right), 2.03\left(\mathrm{~d}, 3 \mathrm{H}, J 5.4 \mathrm{~Hz}, \mathrm{CH}_{2}\right), 2.45(\mathrm{~d}, 1 \mathrm{H}, J 8.1 \mathrm{~Hz}$, $\mathrm{CH}_{2}$ ), 3.18-3.39 (m, 3H, $\left.\mathrm{NCH}_{2}\right), 3.70\left(\mathrm{~s}, 1 \mathrm{H}, \mathrm{NCH}_{2}\right), 4.00$ (t, $\left.2 \mathrm{H}, J 6.6 \mathrm{~Hz}, \mathrm{NCH}_{2}\right), 4.42-4.34(\mathrm{~m}, 1 \mathrm{H}, \mathrm{CH}) ;{ }^{13} \mathrm{C} \mathrm{NMR}$ (75 MHz, $\left.\mathrm{CDCl}_{3}, \mathrm{TMS}\right) \delta 14.3,14.3,22.0,22.8,22.8$, 26.0, 26.0, 27.1, 28.6, 28.6, 29.2, 29.2, 29.4, 29.4, 29.7, 29.7, 29.7, 29.7, 32.0, 32.0, 53.3, 53.3, 67.0, 67.0, 167.9.

\section{CIL $2 f$}

${ }^{1} \mathrm{H}$ NMR $\left(300 \mathrm{MHz}, \mathrm{CDCl}_{3}, \mathrm{TMS}\right) \delta 0.78-0.97(\mathrm{~m}, 6 \mathrm{H}$, $\left.\mathrm{CH}_{3}\right), 1.05-1.40\left(\mathrm{~m}, 36 \mathrm{H}, \mathrm{CH}_{2}\right), 1.48-1.98\left(\mathrm{~m}, 4 \mathrm{H}, \mathrm{CH}_{2}\right)$, $1.98-2.42\left(\mathrm{~s}, 3 \mathrm{H}, \mathrm{CH}_{2}\right), 2.60\left(\mathrm{~s}, 1 \mathrm{H}, \mathrm{CH}_{2}\right), 3.06-3.38(\mathrm{~m}, 3 \mathrm{H}$, $\left.\mathrm{NCH}_{2}\right), 3.64\left(\mathrm{~s}, 1 \mathrm{H}, \mathrm{NCH}_{2}\right), 4.04-4.18\left(\mathrm{~m}, 3 \mathrm{H}, \mathrm{NCH}_{2}, \mathrm{CH}\right)$, 4.20-4.29(m, $1 \mathrm{H}, \mathrm{CH}) ;{ }^{13} \mathrm{C} \mathrm{NMR}\left(75 \mathrm{MHz}, \mathrm{CDCl}_{3}, \mathrm{TMS}\right)$ $\delta$ 14.3, 14.3, 22.3, 22.8, 22.8, 26.0, 26.0, 26.9, 29.2, 29.2, $29.4,29.4,29.4,29.4,29.7,29.7,29.7,29.7,32.0,32.0$, 54.4, 54.4, 67.2, 67.2, 168.1.

\section{General procedure for the aldol reactions}

(L)-Proline (30 mol\%) and the aromatic aldehyde $(1 \mathrm{mmol})$ were added in the acetone $(3 \mathrm{~mL})$; after stirred for $1 \mathrm{~min}$, chiral ionic liquids (CILs) $(7.5 \mathrm{~mol} \%$ ) were added and the reaction mixture was stirred at room temperature $\left(25{ }^{\circ} \mathrm{C}\left( \pm 3{ }^{\circ} \mathrm{C}\right)\right)$ for $24 \mathrm{~h}$. The acetone was evaporated, the residue was extracted with diethyl ether $(3 \mathrm{~mL}$, three times), separated diethyl ether layer was collected, the residues were dried under vacuum at $40{ }^{\circ} \mathrm{C}$ during $5 \mathrm{~h}$ for the next cycle, the collected solvent was evaporated and the residues purified by chromatography on $\mathrm{SiO}_{2}$-column (Petroleum ether:EtOAc = 3:1). All reaction products had the physical constants and NMR spectra in accord with the published data. The petroleum ether used was of boiling range $60-80{ }^{\circ} \mathrm{C}$. Evaporation of solvent was performed at reduced pressure at $25( \pm 3)^{\circ} \mathrm{C}$.

\section{Supplementary Information}

Supplementary data are available free of charge at http://jbcs.sbq.org.br as PDF file.

\section{Acknowledgments}

We thank Dr. Zhi-Yong Xue (Wuhan University, Wuhan, China) for help in the HPLC analysis and the National Natural Science Foundation of China (Grant Number: 20972120).

\section{References}

1. Welton, T.; Chem. Rev. 1999, 99, 2071.

2. Ranke, J.; Stolte, S.; Stormann, R.; Arning, J.; Jastorff, B.; Chem. Rev. 2007, 107, 2183.

3. Howarth, J.; Hanlon, K.; Fayne, D.; McCormac, P.; Tetrahedron Lett. 1997, 38, 3097.

4. Earle, M. J.; McCormac, P. B.; Seddon, K. R.; Green Chem. 1999, 1, 23.

5. Pastre, J. C.; Génisson, Y.; Saffon, N.; Dandurand, J.; Correia, C. R. D.; J. Braz. Chem. Soc. 2010, 21, 821.

6. Dupont, J.; Suarez, P. A. Z.; Umpierre, A. P.; Souza, R. F.; J. Braz. Chem. Soc. 2000, 11, 293.

7. Pilissão, C.; Carvalho, P. O.; Nascimento, M. G.; J. Braz. Chem. Soc. 2010, 21, 973.

8. Narayanaperumal, S.; Gul, K.; Kawasoko, C. Y.; Singh, D.; Dornelles, L.; Rodrigues, O. E. D.; Braga, A. L.; J. Braz. Chem. Soc. 2010, 21, 2079.

9. Yu, W.; Zhang, H.; Zhang, L.; Zhou, X.; Aust. J. Chem. 2010, 63, 299.

10. Geary, L. M.; Hultin, P. G.; Tetrahedron: Asymmetry 2009, 20, 131.

11. Kimball, D. B.; Silks, L. A.; Curr. Org. Chem. 2006, 10, 1975.

12. North, M.; Pizzato, F.; Villuendas, P.; ChemSusChem 2009, 2 , 862.

13. Mahrwald, R.; Chem. Rev. 1999, 99, 1095. 
14. Luo, S.; Mi, X.; Zhang, L.; Liu, X. H.; Cheng, J. P.; Tetrahedron 2007, 63, 1923.

15. List, B.; Lerner, R. A.; Barbas, C. F.; J. Am. Chem. Soc. 2000, 122, 2395.

16. Tang, Z.; Jiang, F.; Yu, L. T.; Cui, X.; Gong, L. Z.; Mi, A. Q.; Jiang, Y. Z.; Wu, Y. D.; J. Am. Chem. Soc. 2003, 125, 5262.

17. Sato, K.; Kuriyama, M.; Shimazaw, R.; Morimoto, T.; Kakiuchi, K.; Shirai, R.; Tetrahedron Lett. 2008, 49, 2402.

18. Sakthivel, K.; Notz, W.; Bui, T.; Barbas, C. F.; J. Am. Chem. Soc. 2001, 123, 5260.

19. Jia, Y. N.; Wu, F. C.; Ma, X.; Zhu, G. J.; Da, C. S.; Tetrahedron Lett. 2009, 50, 3059.

20. Guo, H. M.; Niu, H. Y.; Xue, M. X.; Guo, Q. X.; Cun, L. F.; Mi, A. Q.; Jiang, Y. Z.; Wang, J. J.; Green. Chem. 2006, 8, 682.
21. Kotrusz, P.; Kmentova, I.; Gotov, B.; Toma, S.; Solcaniova, E.; Chem. Commun. 2002, 2510.

22. Shah, J.; Blumenthal, H.; Yacob, Z.; Liebscher, J.; Adv. Synth. Catal. 2008, 350, 1267.

23. Reddy, K. R.; Chakrapani, L.; Ramani, T.; Rajasekhar, C. V.; Synth. Commun. 2007, 37, 4301.

24. Qian, Y.; Zheng, X.; Wang, Y.; Eur. J. Org. Chem. 2010, 19, 3672 .

25. Movassaghi, M.; Jacobsen, E. N.; Science 2002, 298, 1904.

Submitted: February 21, 2011

Published online: June 21, 2011 


\title{
Novel Chiral Ionic Liquid (CIL) Assisted Selectivity Enhancement to (L)-Proline Catalyzed Asymmetric Aldol Reactions
}

\author{
Long Zhang, Haibo Zhang, * Huadong Luo, Xiaohai Zhou* and Gongzhen Cheng \\ College of Chemistry, Wuhan University, 430072, P. R. China
}

General remarks

${ }^{1} \mathrm{H}$ NMR spectra were recorded on a VARIAN Mercury $300 \mathrm{MHz}$ spectrometer or VARIAN Mercury $600 \mathrm{MHz}$ spectrometer. Chemical shifts are reported in ppm with the TMS as internal standard. The data are reported as ( $\mathrm{s}=$ singlet, $\mathrm{d}=$ doublet, $\mathrm{t}=$ triplet, $\mathrm{q}=$ quartet, $\mathrm{m}=$ multiplet or unresolved, brs = broad singlet, coupling constant(s) in $\mathrm{Hz}$, integration). ${ }^{13} \mathrm{C}$ NMR spectra were recorded on a VARIAN Mercury $75 \mathrm{MHz}$ or VARIAN
Mercury $125 \mathrm{MHz}$ spectrometer. Chemical shifts are reported in ppm with the internal chloroform signal at $77.0 \mathrm{ppm}$ as a standard. Commercially obtained reagents were used without further purification. All reactions were monitored by TLC with silica gel-coated plates. Enantiomeric ratios were determined by HPLC, using a chiralpak AS-H column, a chiralpak AD-H column or a chiralcel OD-H column with hexane and $i$-PrOH as solvents. The configurations were assigned by comparison of the $t_{R}$ with the reported data. ${ }^{1,2}$

\section{Characterization of CILs}

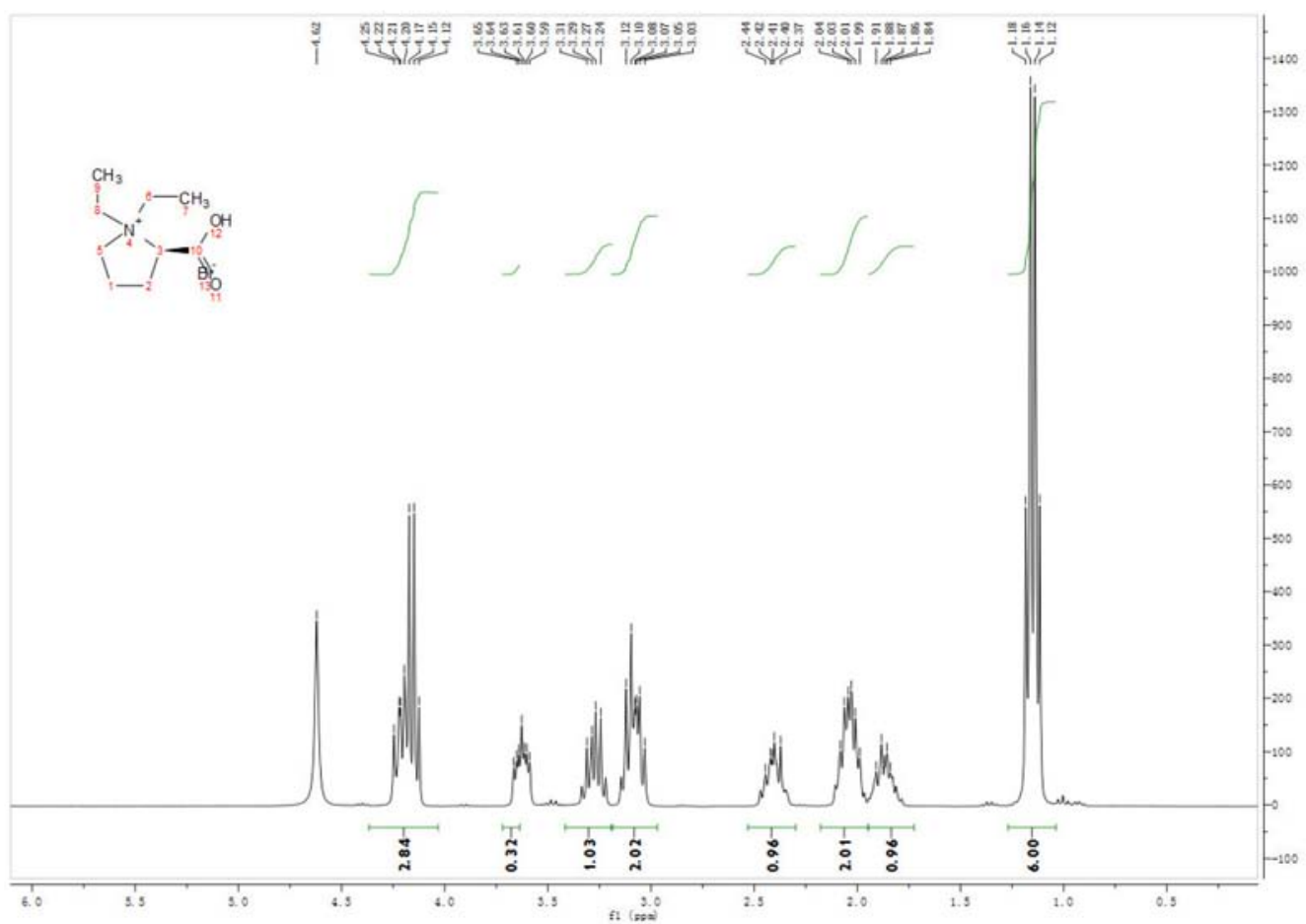

Figure S1. ${ }^{1} \mathrm{H}$ NMR (300 MHZ, $\left.\mathrm{D}_{2} \mathrm{O}\right)$ spectrum of $\mathbf{2 a}$. 


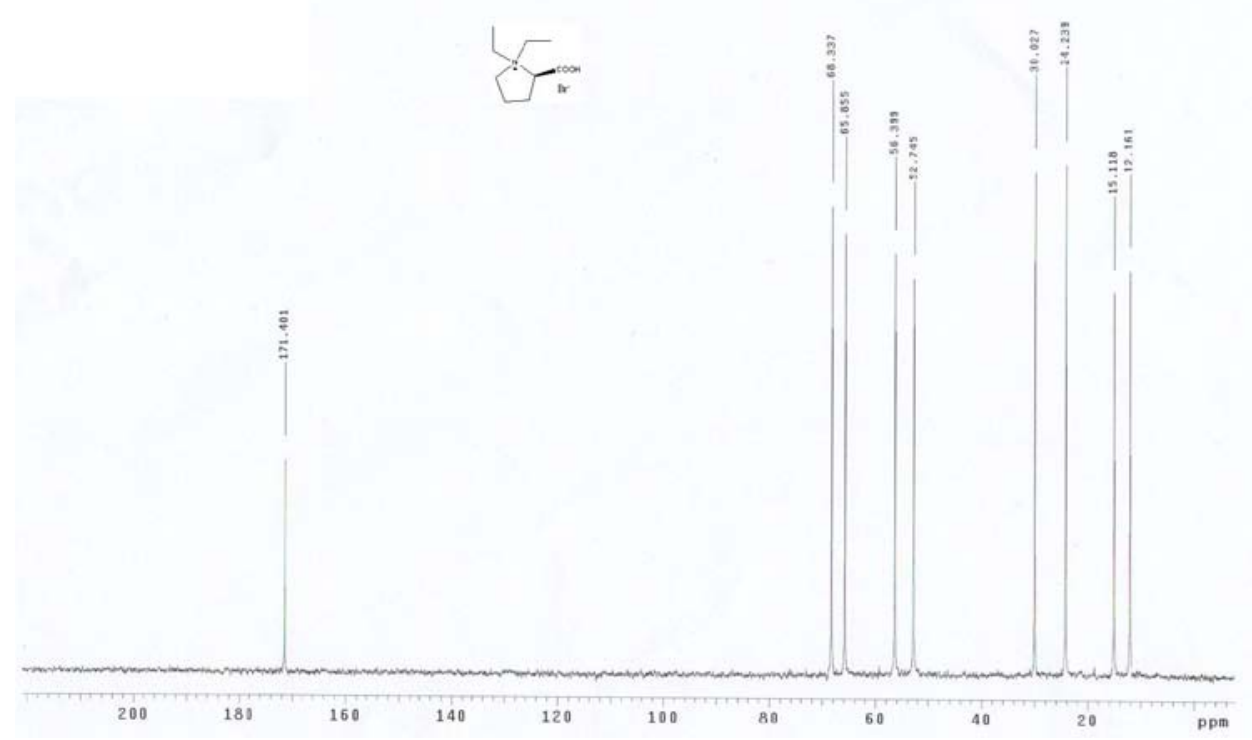

Figure S2. ${ }^{13} \mathrm{C}$ NMR $\left(75 \mathrm{MHZ}, \mathrm{D}_{2} \mathrm{O}\right)$ spectrum of $\mathbf{2 a}$.

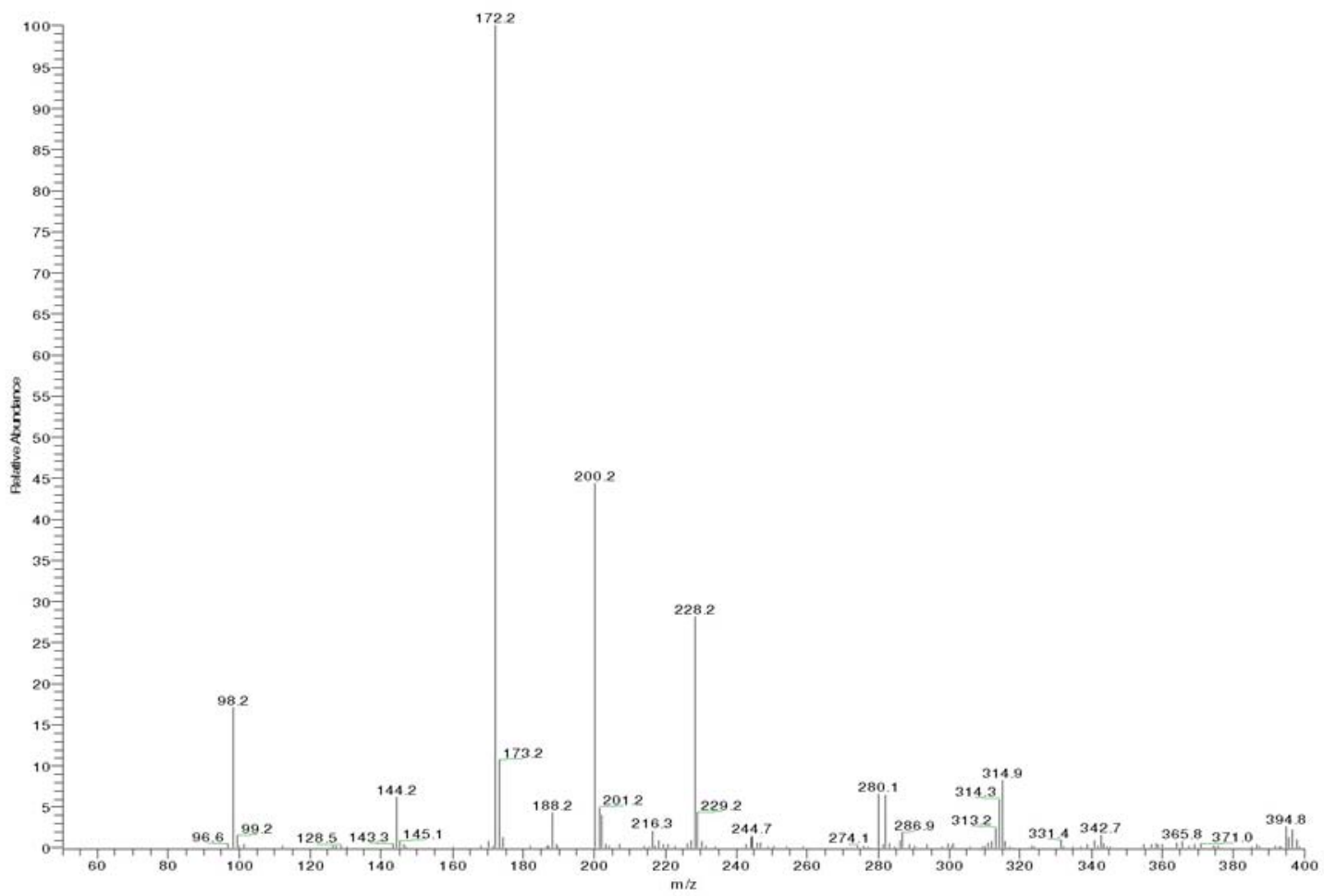

Figure S3. ESI-Mass spectrum of 2 a. 


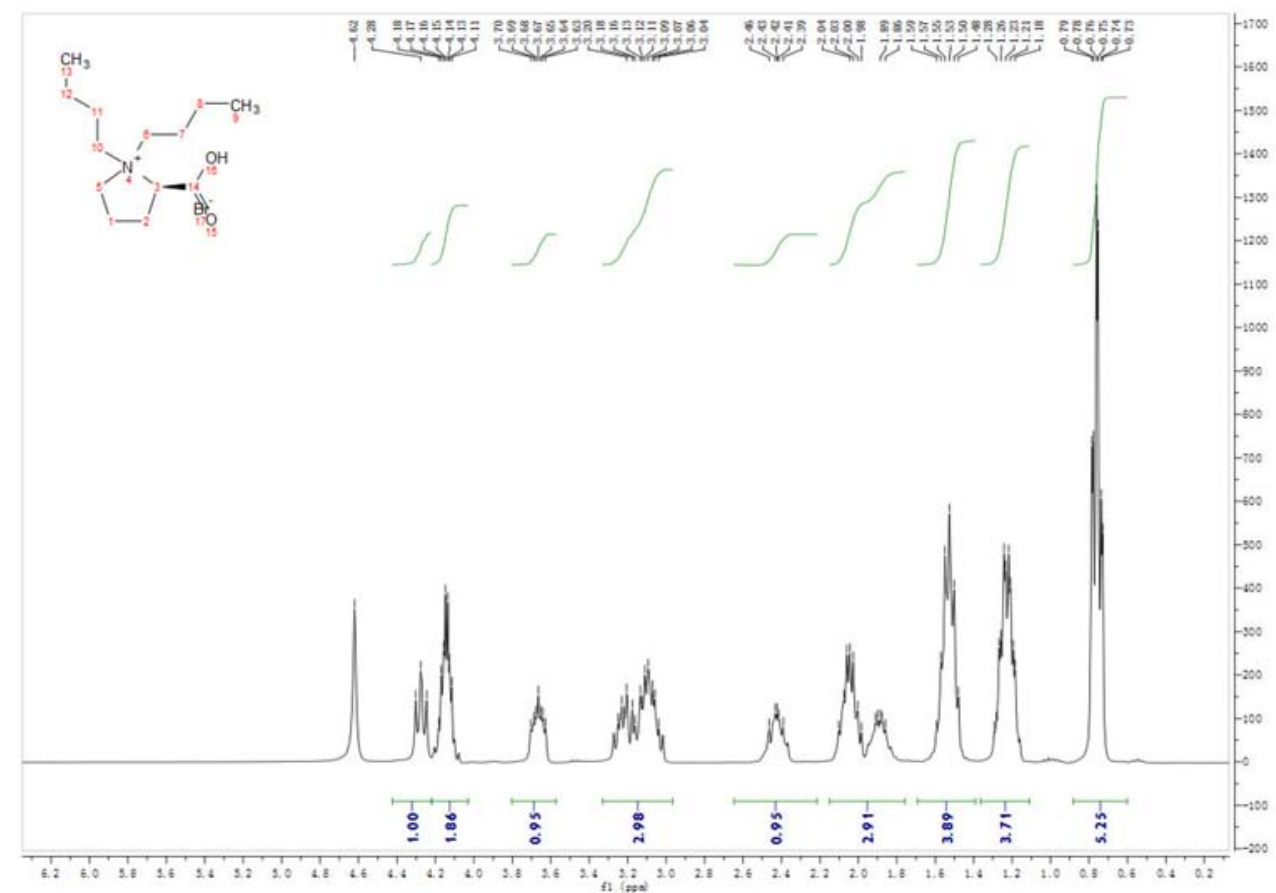

Figure S4. ${ }^{1} \mathrm{H}$ NMR (300 MHZ, $\mathrm{D}_{2} \mathrm{O}$ ) spectrum of $\mathbf{2 b}$.
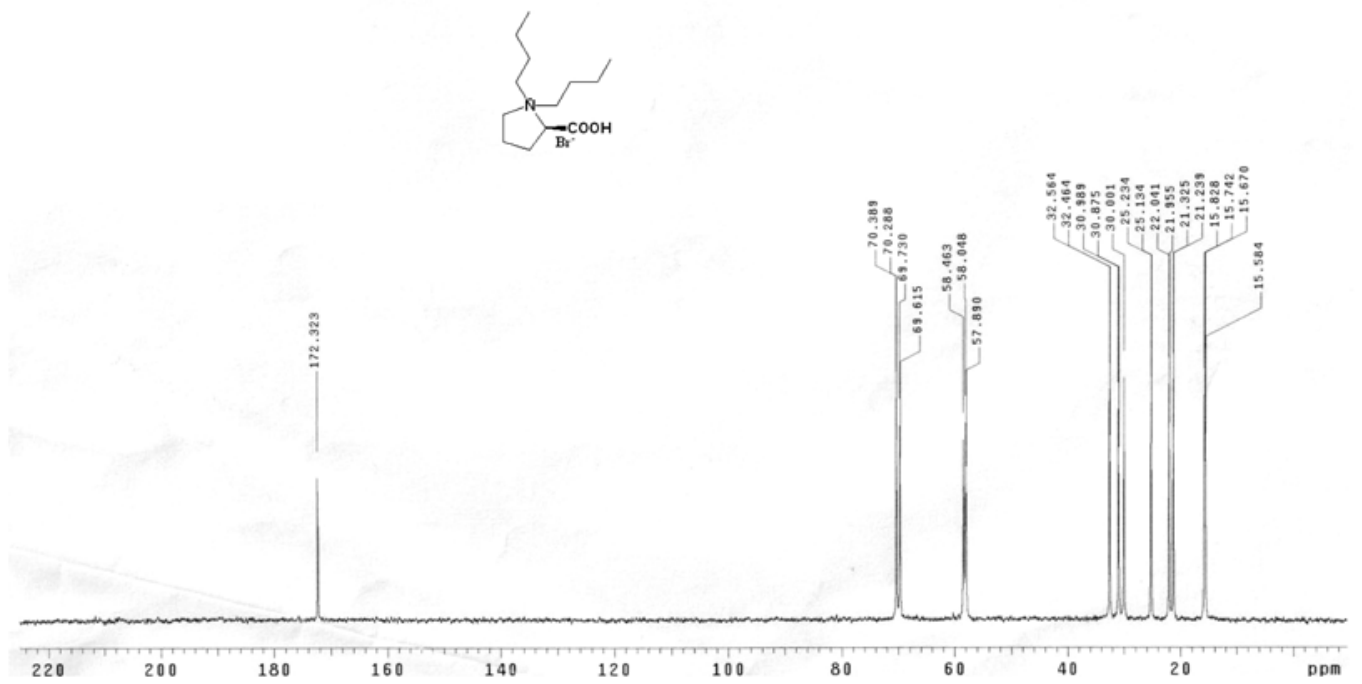

Figure S5. ${ }^{13} \mathrm{C}$ NMR $\left(75 \mathrm{MHZ}, \mathrm{D}_{2} \mathrm{O}\right)$ spectrum of $\mathbf{2 b}$. 


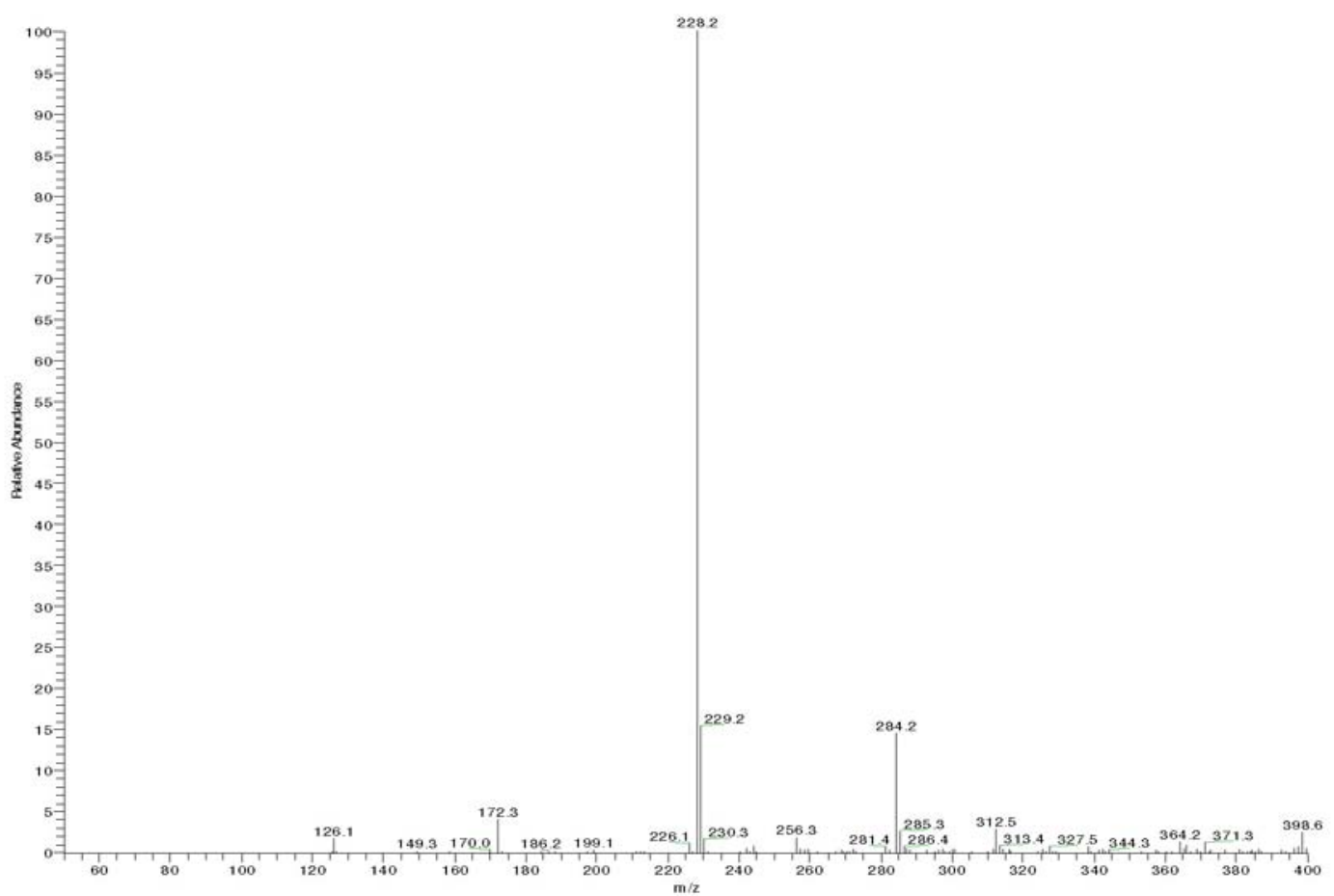

Figure S6. ESI-Mass spectrum of $\mathbf{2 b}$.

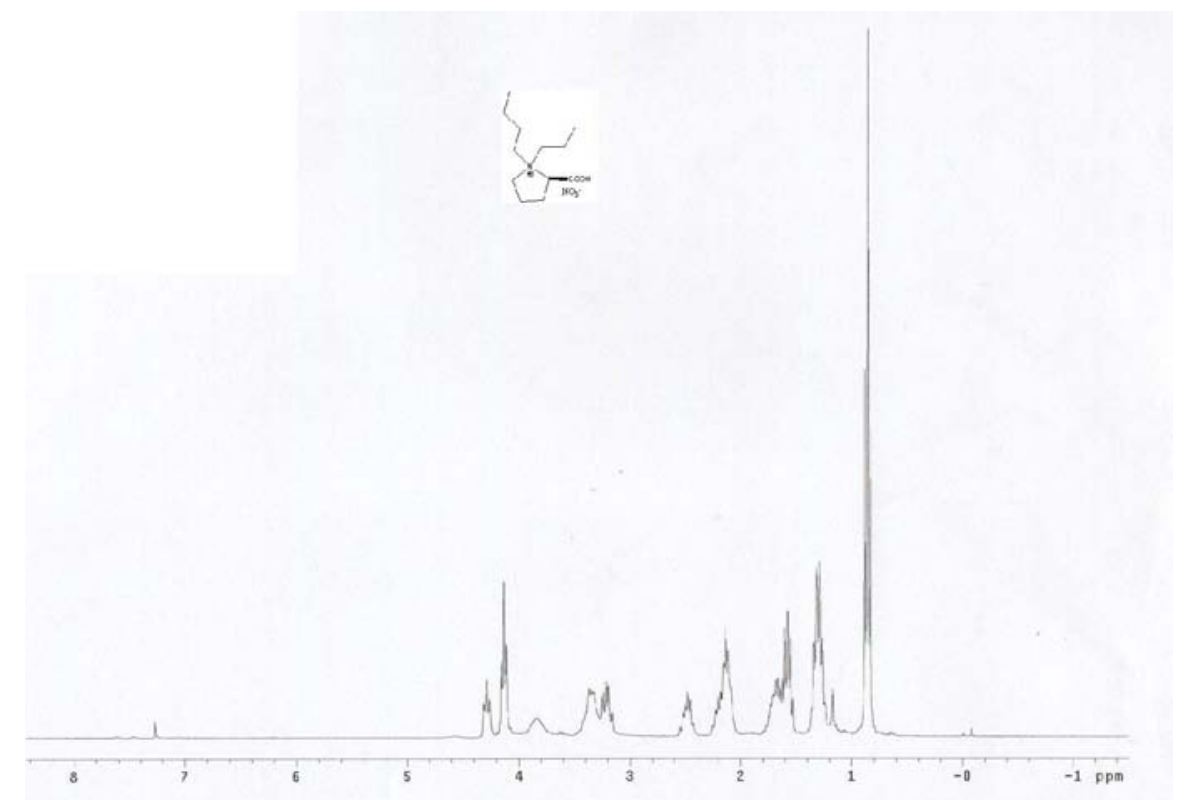

Figure S7. ${ }^{1} \mathrm{H}$ NMR (300 MHZ, $\mathrm{CDCl}_{3}$ ) spectrum of 3a. 
Vol. 22, No. 9, 2011

Chang et al.

SF

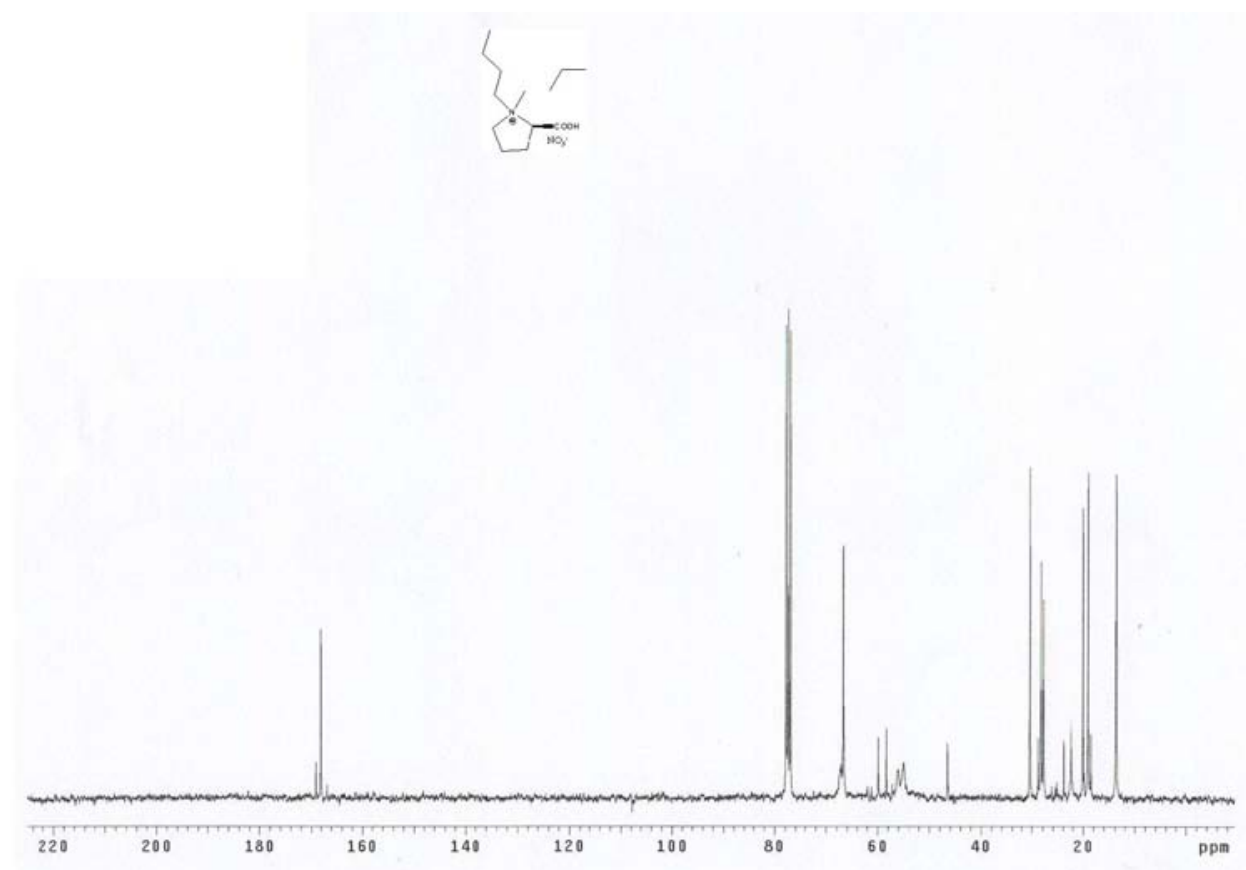

Figure S8. ${ }^{13} \mathrm{C} \mathrm{NMR}\left(75 \mathrm{MHZ}, \mathrm{CDCl}_{3}\right.$ ) spectrum of Ba. 

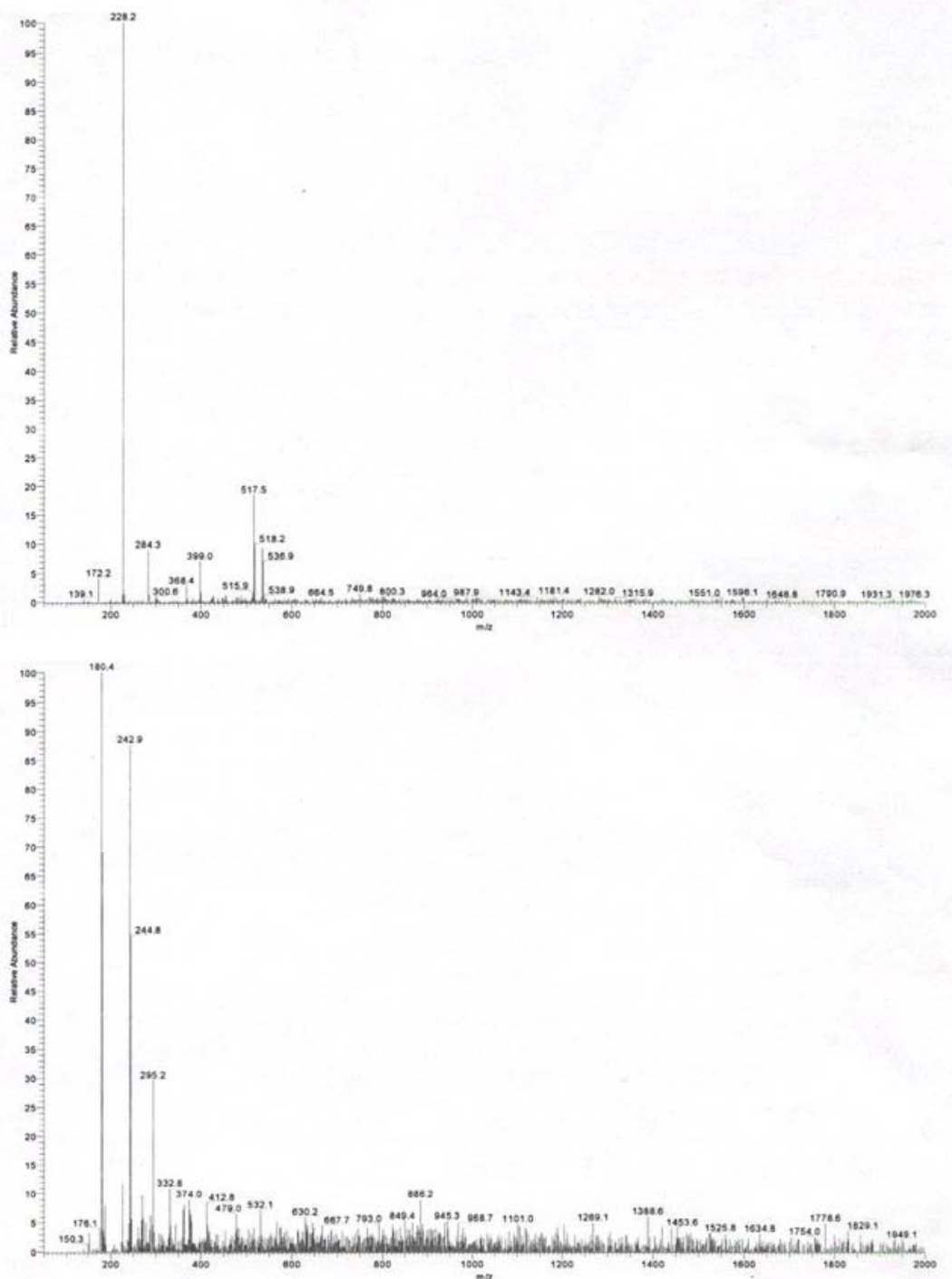

Figure S9. ESI-Mass spectrum of $\mathbf{3 a}$. 


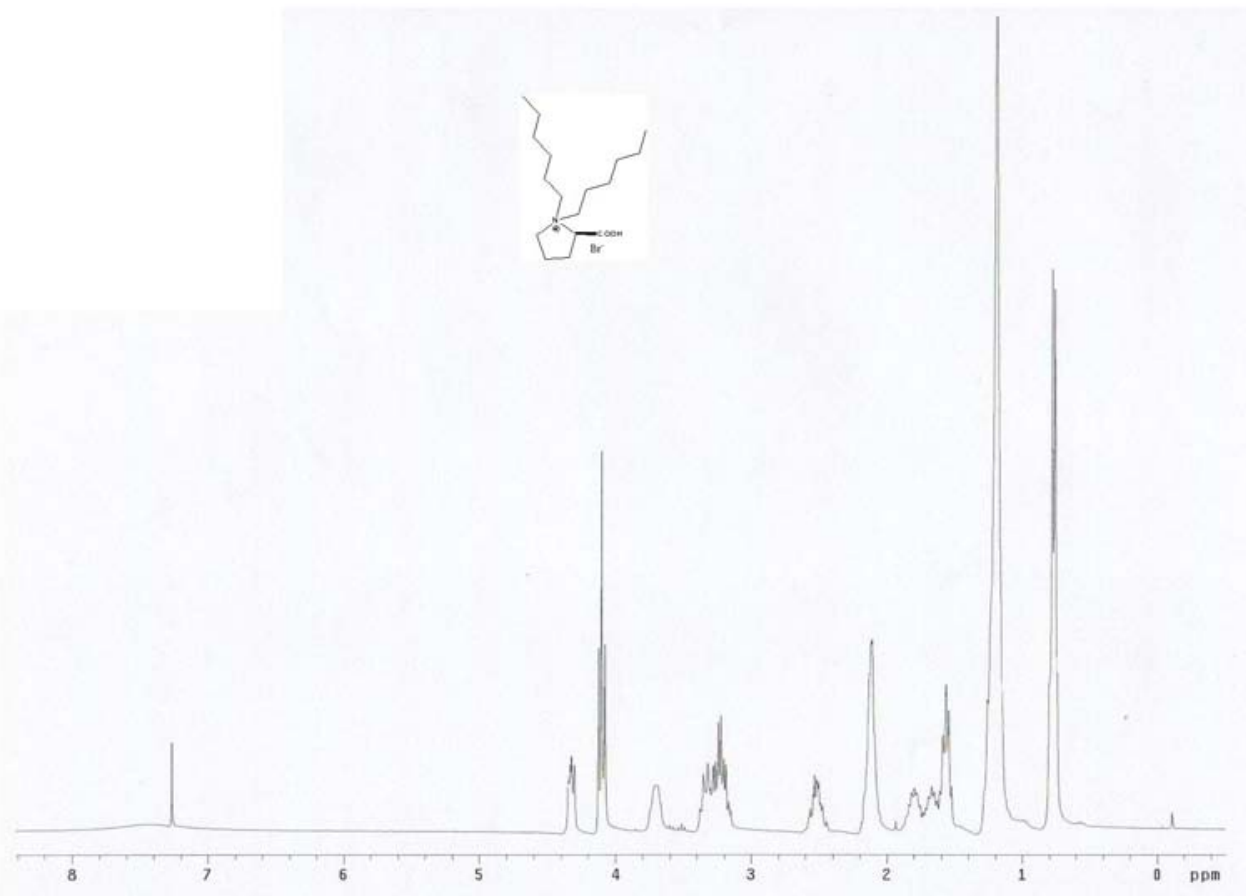

Figure S10. ${ }^{1} \mathrm{H}$ NMR (300 $\mathrm{MHZ}, \mathrm{CDCl}_{3}$ ) spectrum of 2c.

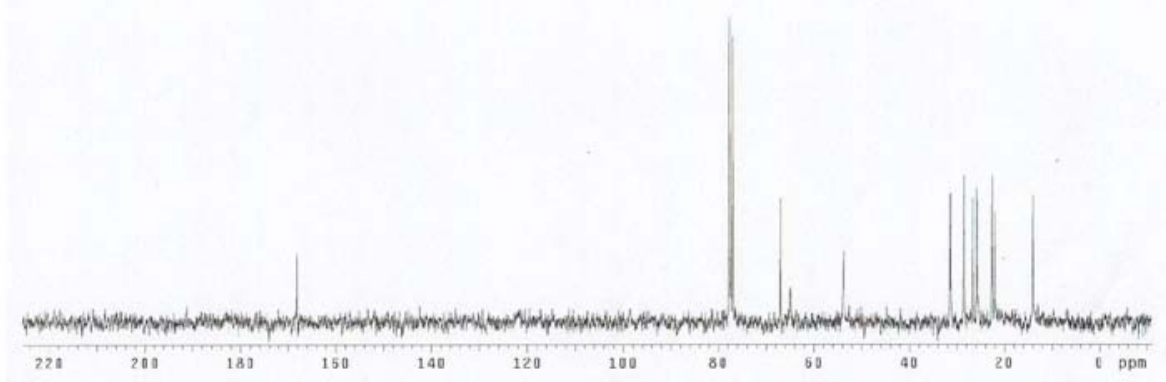

Figure S11. ${ }^{13} \mathrm{C}$ NMR (75 MHZ, $\mathrm{CDCl}_{3}$ ) spectrum of 2c. 


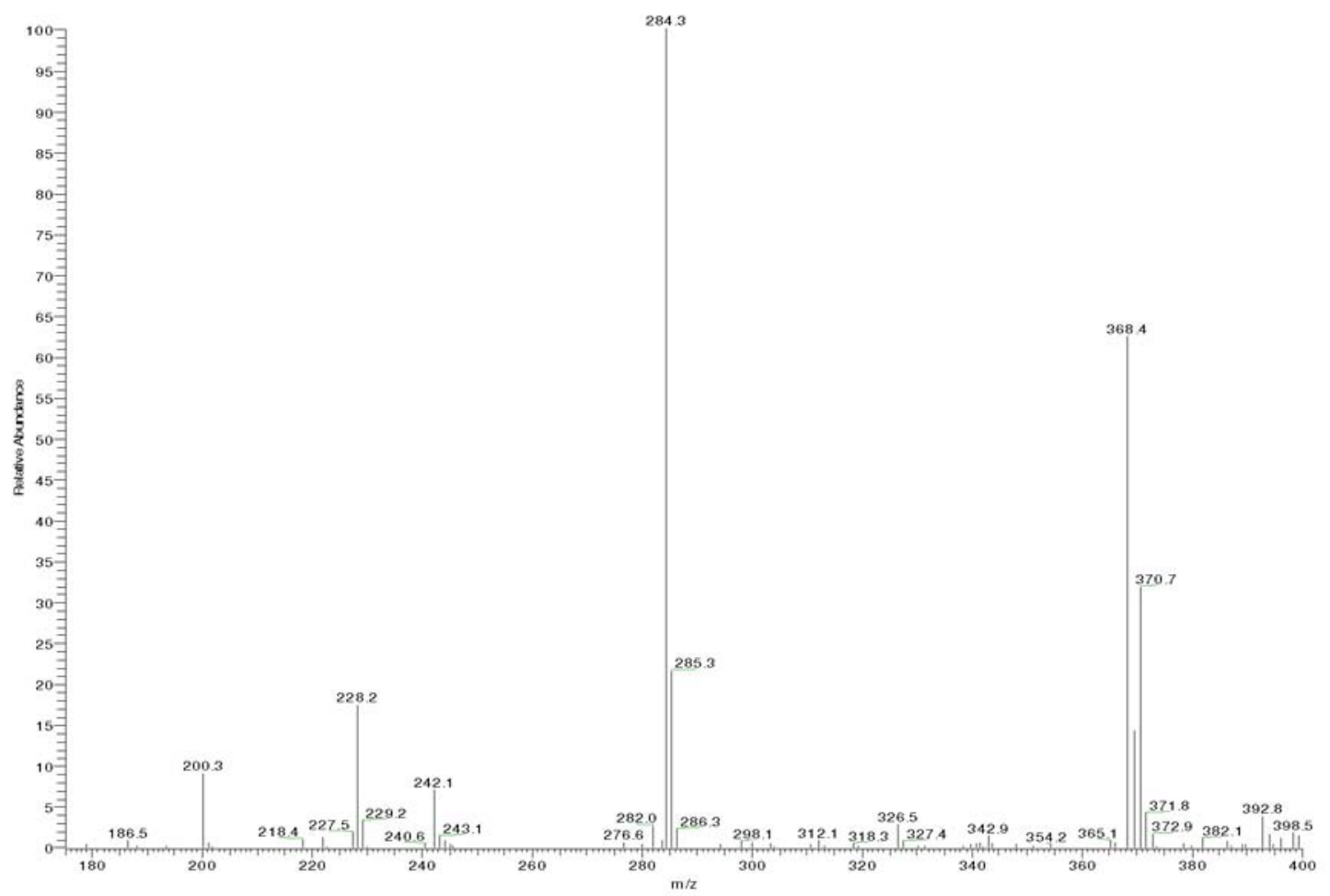

Figure S12. ESI-Mass spectrum of 2 c.

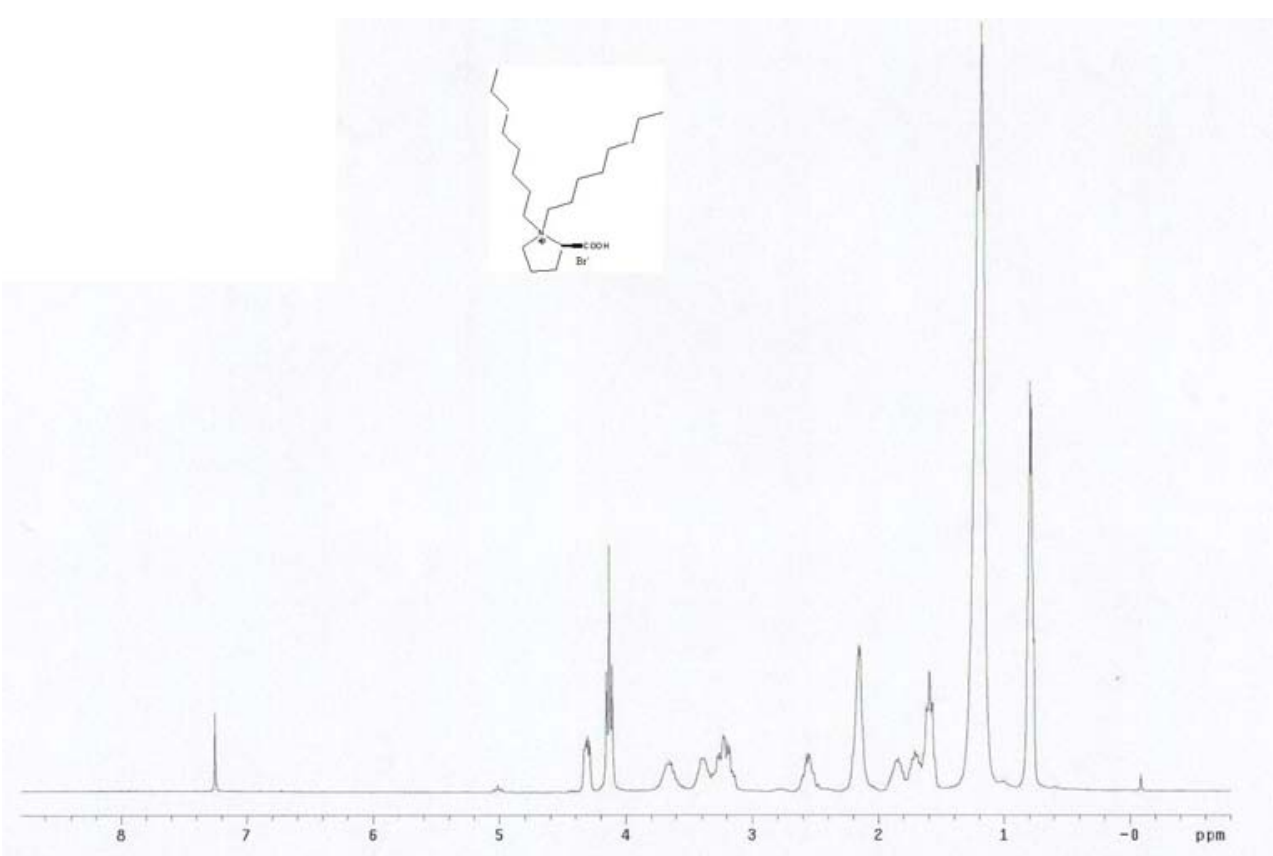

Figure S13. ${ }^{1} \mathrm{H}$ NMR (300 MHZ, $\mathrm{CDCl}_{3}$ ) spectrum of 2d. 


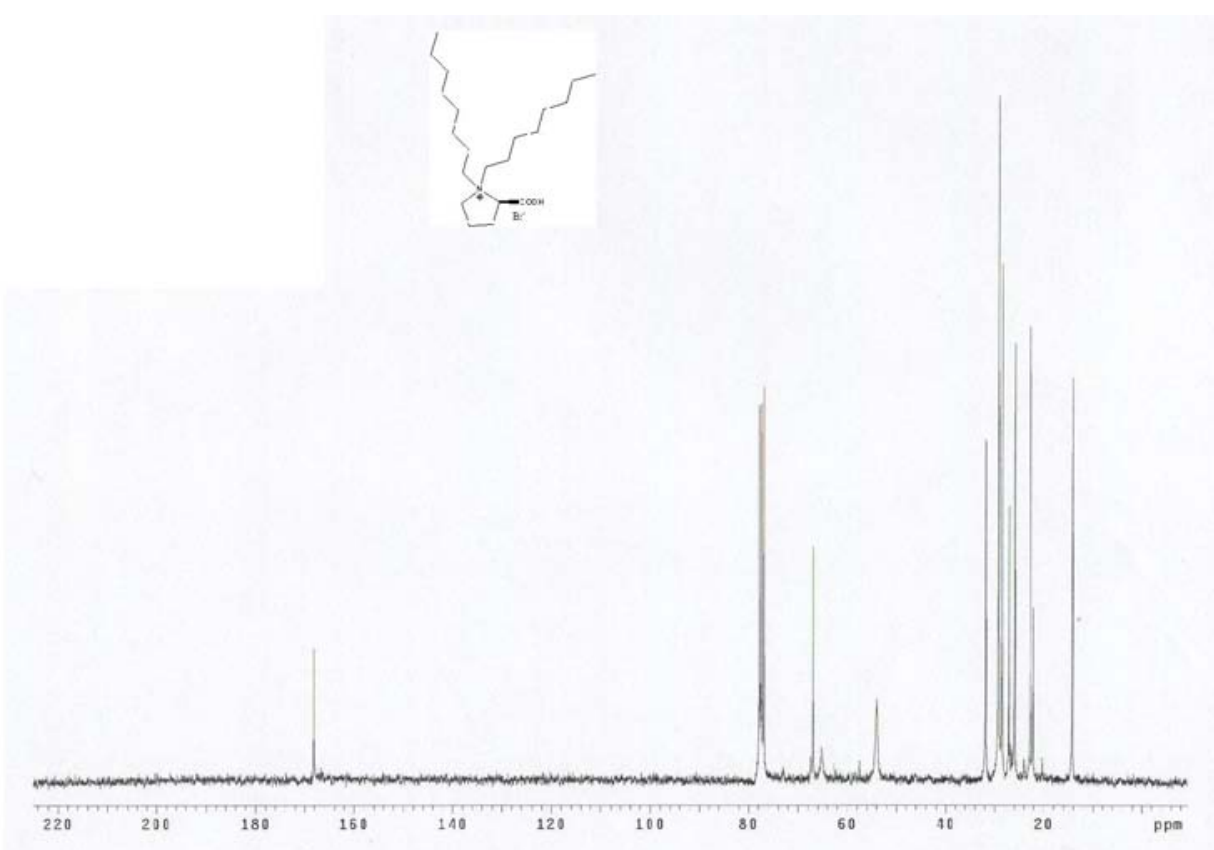

Figure S14. ${ }^{13} \mathrm{C} \mathrm{NMR}\left(75 \mathrm{MHZ}, \mathrm{CDCl}_{3}\right.$ ) spectrum of $\mathbf{2 d}$.

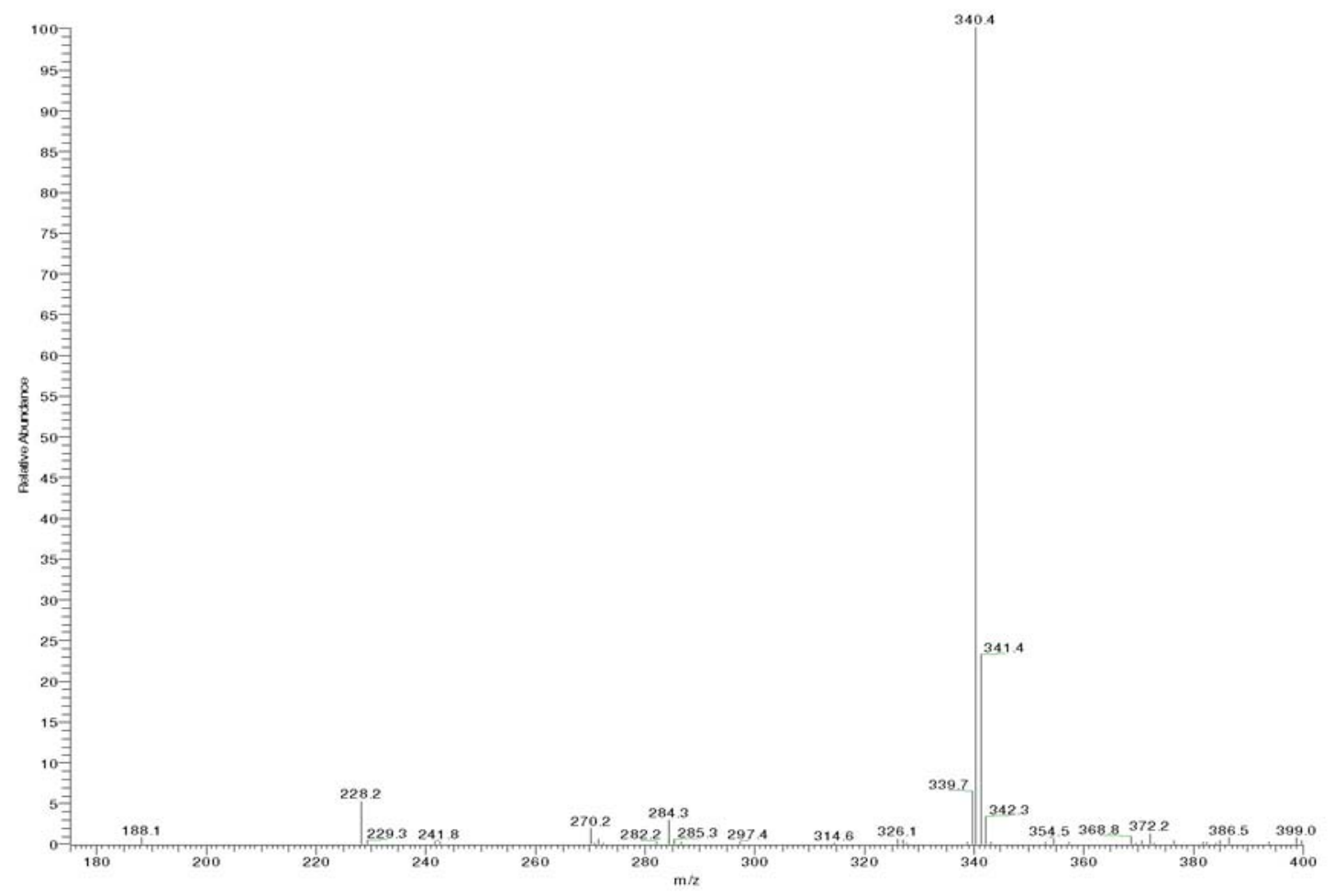

Figure S15. ESI-Mass spectrum of 2d. 


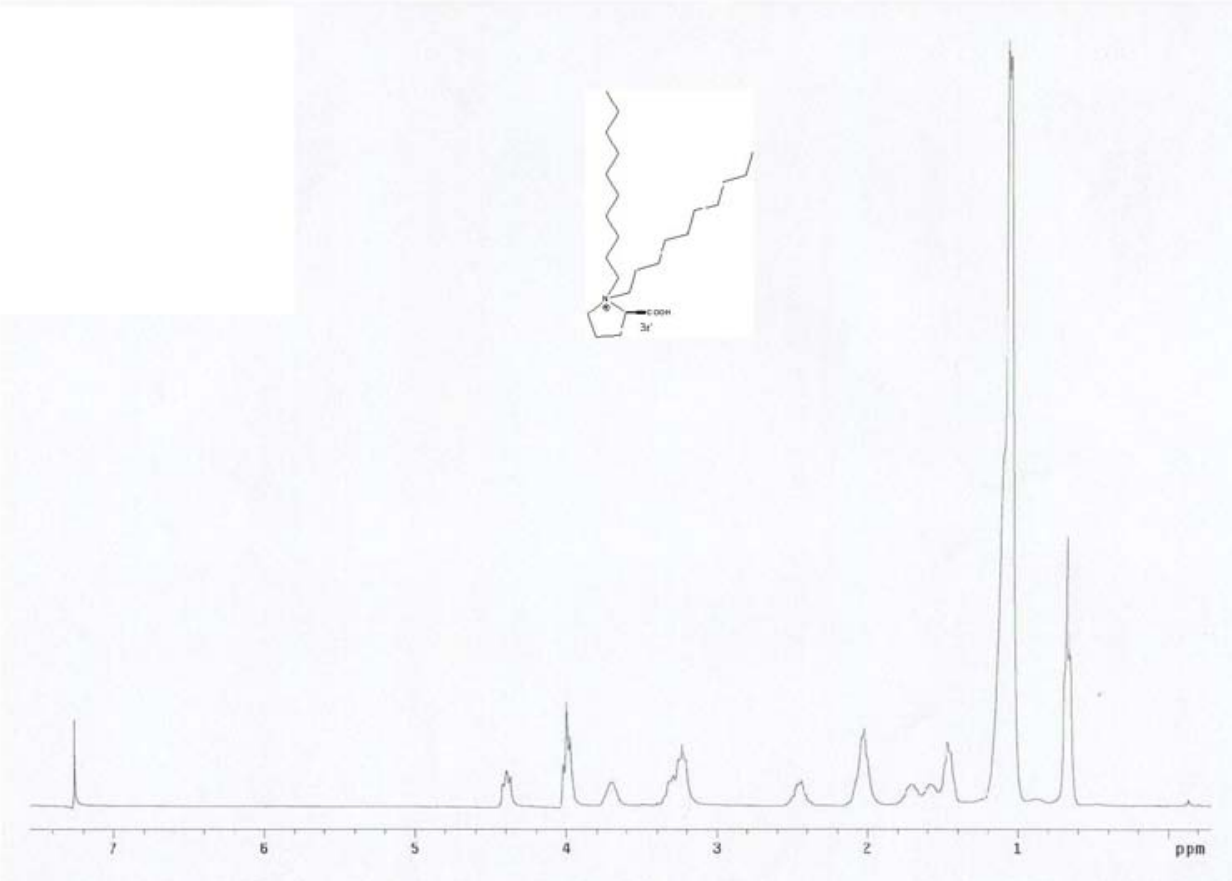

Figure S16. ${ }^{1} \mathrm{H}$ NMR $\left(300 \mathrm{MHZ}, \mathrm{CDCl}_{3}\right)$ spectrum of $2 \mathbf{e}$.
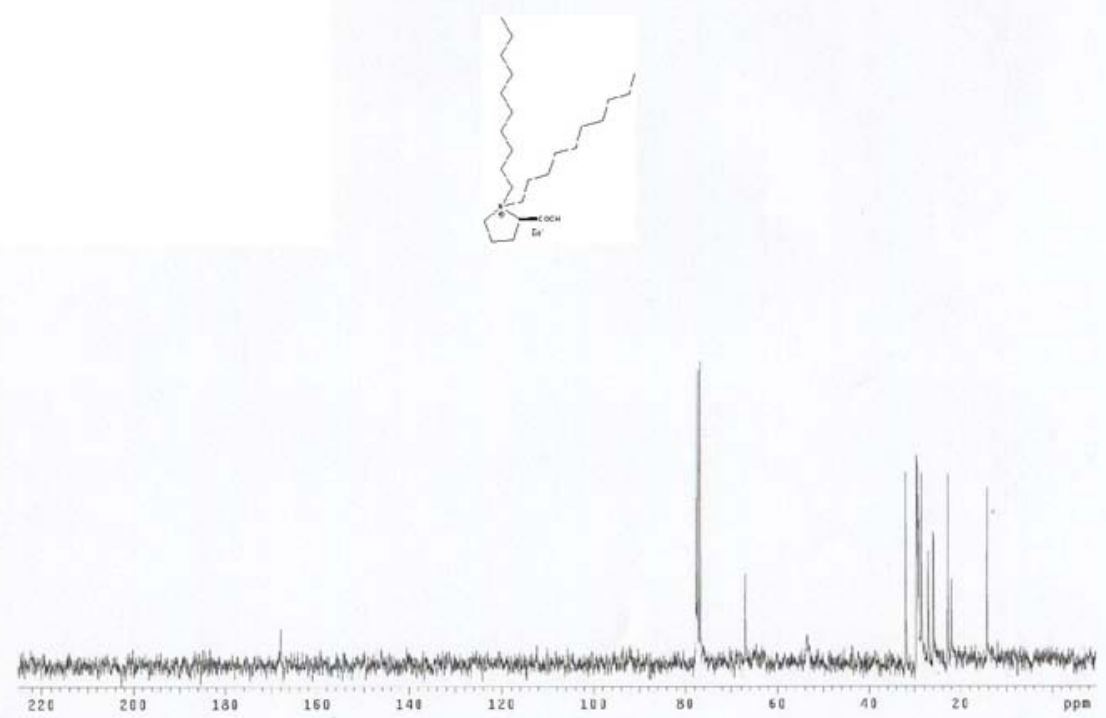

Figure S17. ${ }^{13} \mathrm{C}$ NMR (75 $\mathrm{MHZ}, \mathrm{CDCl}_{3}$ ) spectrum of $\mathbf{2 e}$. 


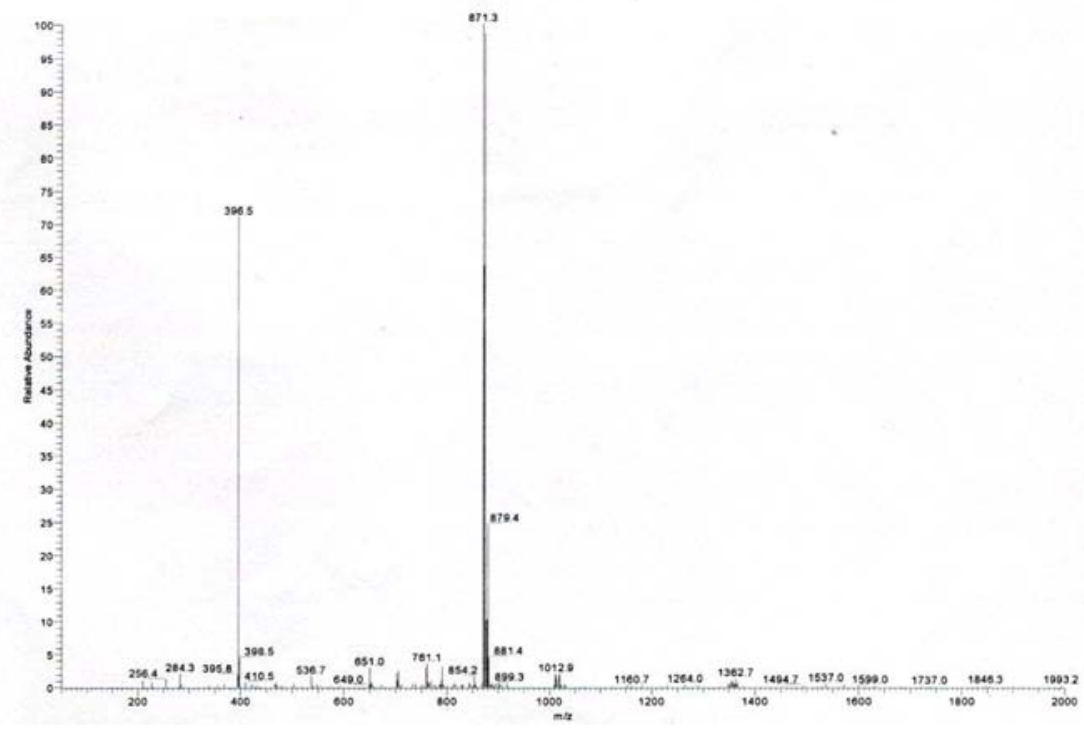

Figure S18. ESI-Mass spectrum of $\mathbf{2 e .}$

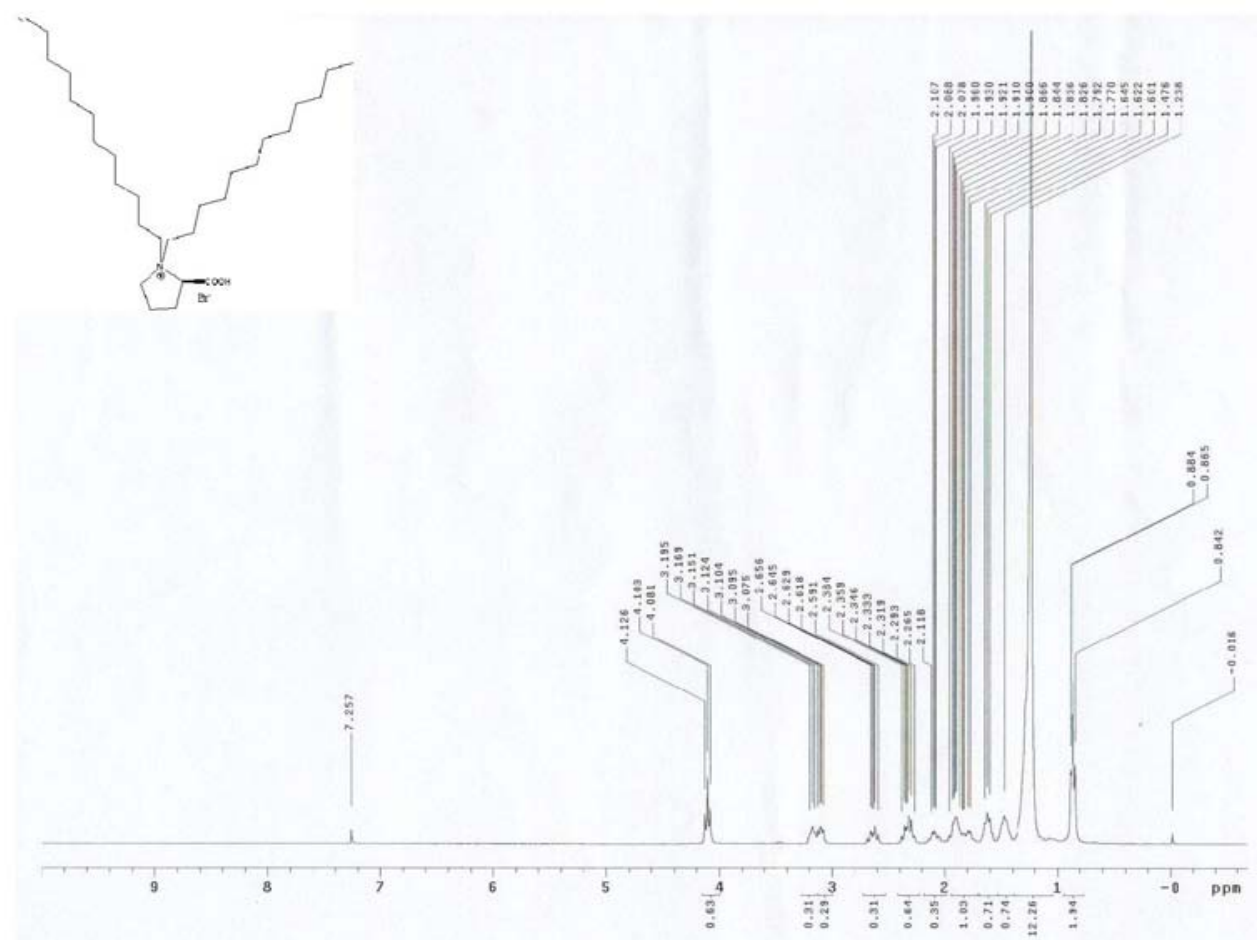

Figure S19. ${ }^{1} \mathrm{H} \mathrm{NMR}\left(300 \mathrm{MHZ}, \mathrm{CDCl}_{3}\right.$ ) spectrum of $\mathbf{2 f}$. 


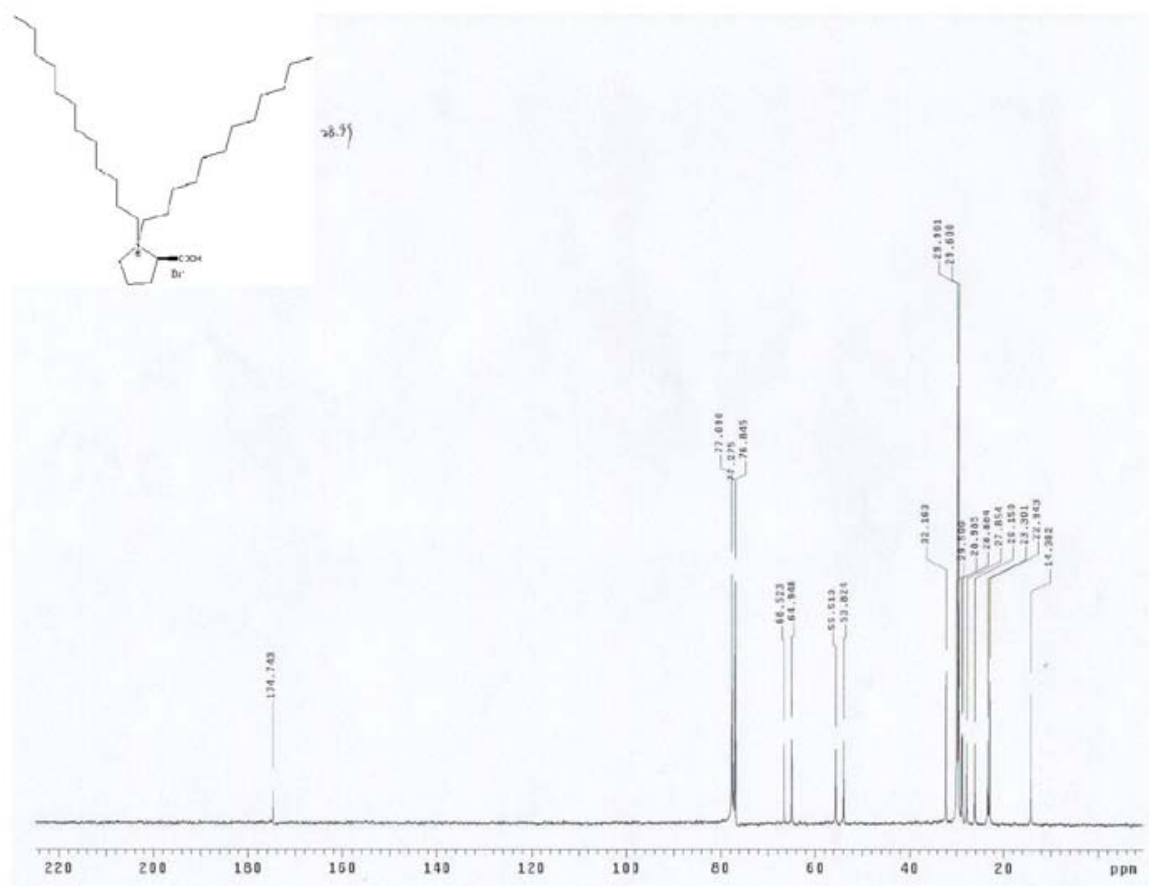

Figure S20. ${ }^{13} \mathrm{C}$ NMR $\left(75 \mathrm{MHZ}, \mathrm{CDCl}_{3}\right)$ spectrum of $2 \mathbf{f}$.

\section{Characterization of aldol products}

\section{NMR data}

All available reagents and solvents were used without further purification. ${ }^{1} \mathrm{H}$ NMR and ${ }^{13} \mathrm{C}$ NMR spectra were conducted on Mercury VX-300 (Varian 300 MHz) or Unity-Inova 600 (Varian 600 MHz) spectrometer. Chemical shifts are expressed in ppm use TMS as internal standard and coupling constants are reported in Hz.

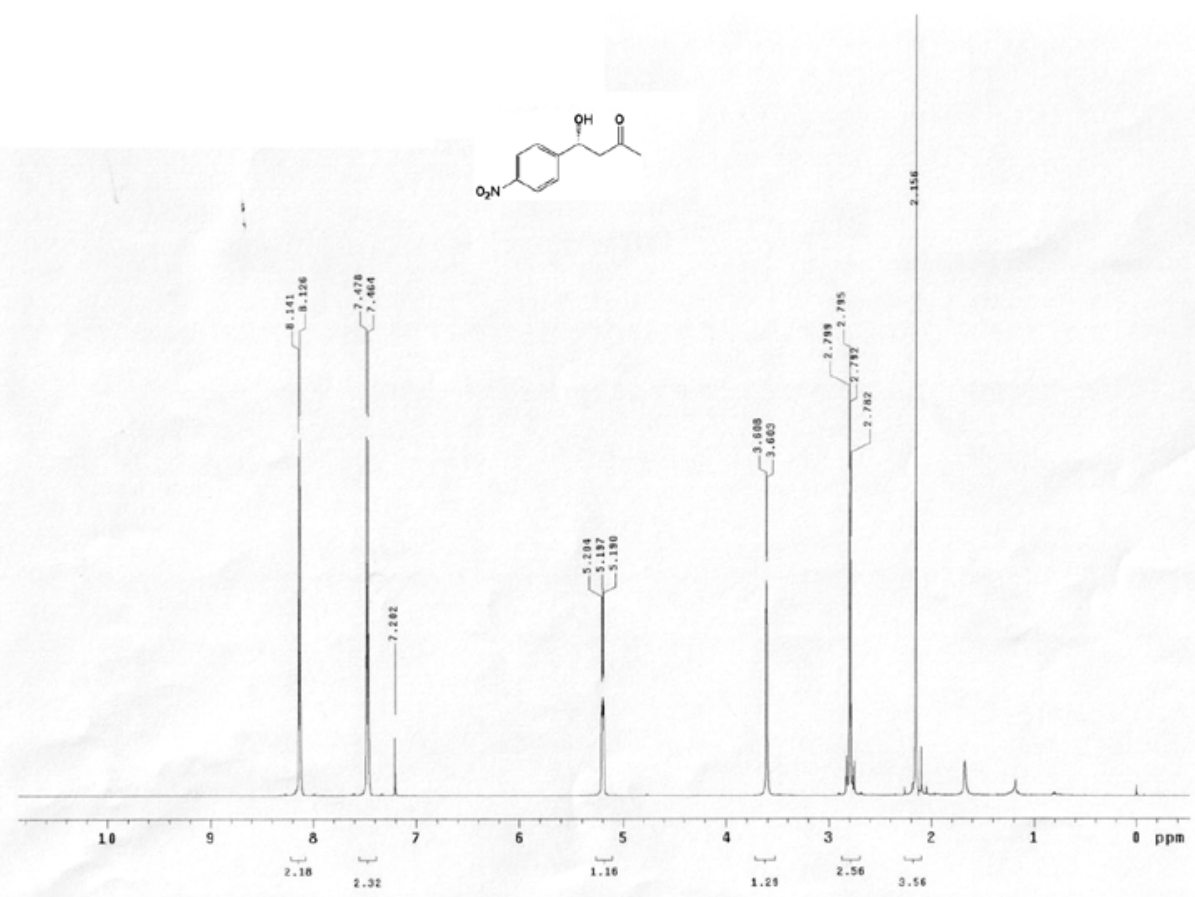

Figure S21. ${ }^{1} \mathrm{H}$ NMR $\left(600 \mathrm{MHZ}, \mathrm{CDCl}_{3}\right)$ spectrum of $\mathbf{5 a}$. 


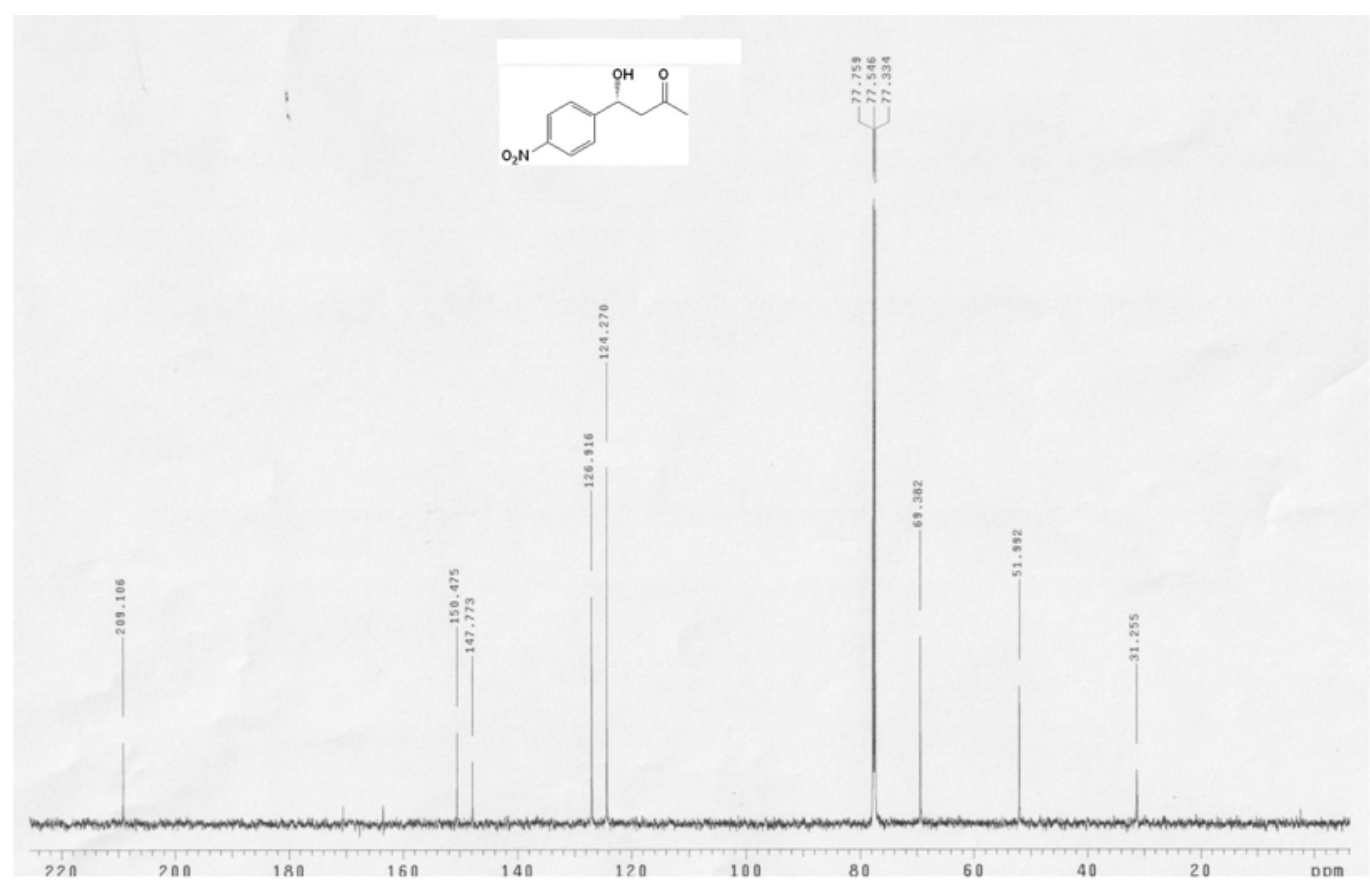

Figure S22. ${ }^{13} \mathrm{C}$ NMR (125 $\left.\mathrm{MHZ}, \mathrm{CDCl}_{3}\right)$ spectrum of $\mathbf{5 a}$.

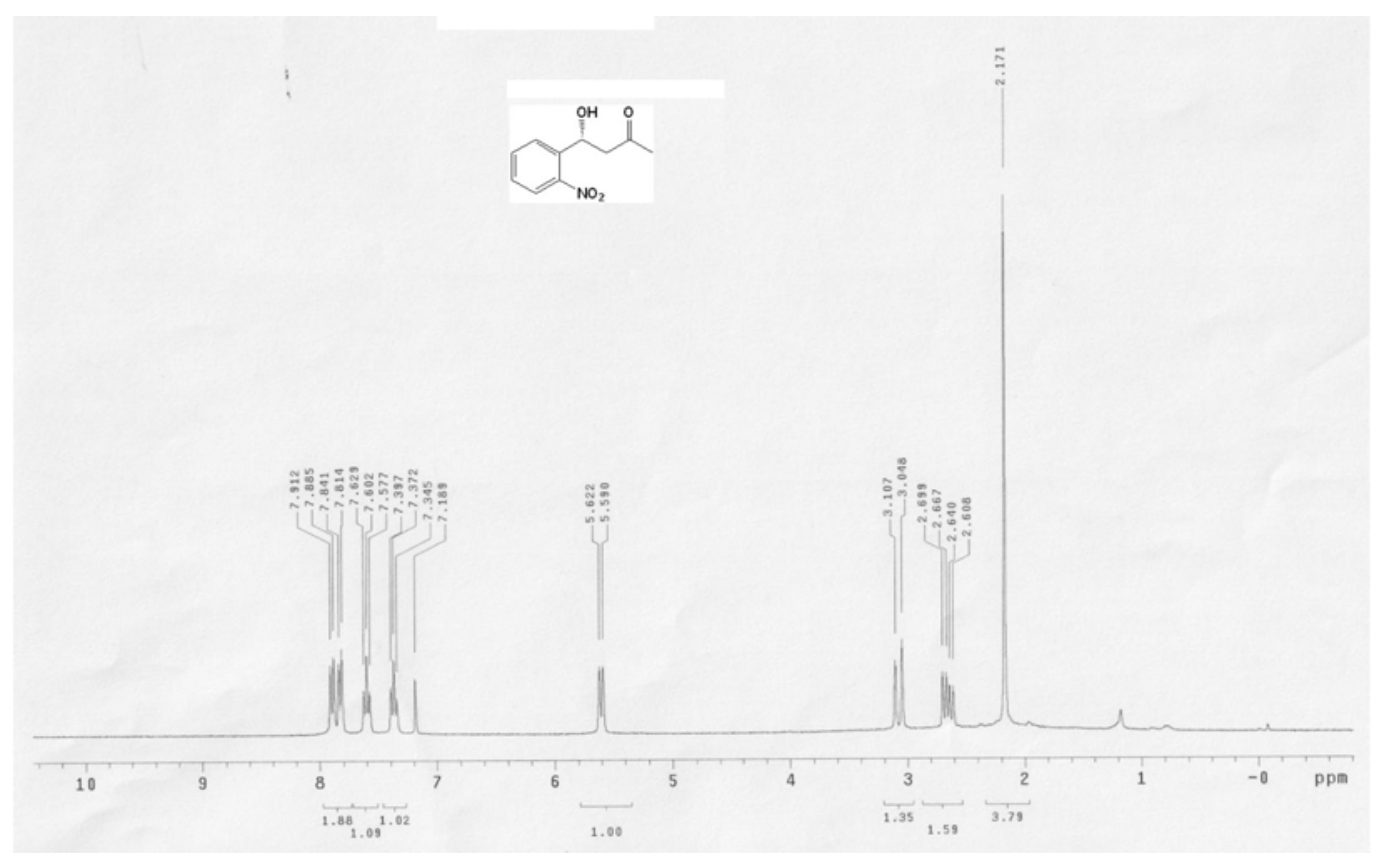

Figure S23. ${ }^{1} \mathrm{H}$ NMR (300 $\left.\mathrm{MHZ}, \mathrm{CDCl}_{3}\right)$ spectrum of $\mathbf{5 b}$. 


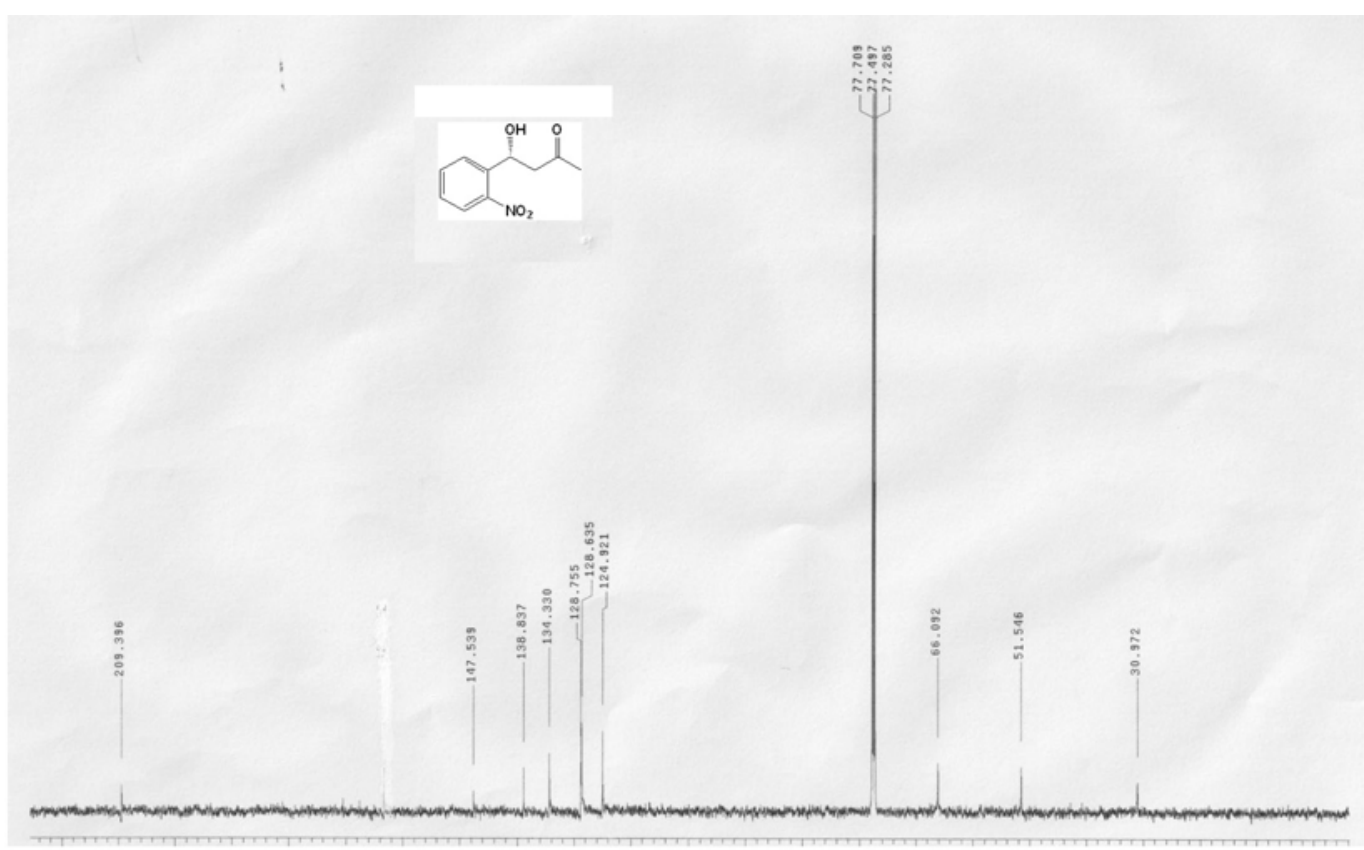

Figure S24. ${ }^{13} \mathrm{C}$ NMR $\left(125 \mathrm{MHZ}, \mathrm{CDCl}_{3}\right)$ spectrum of $\mathbf{5 b}$.

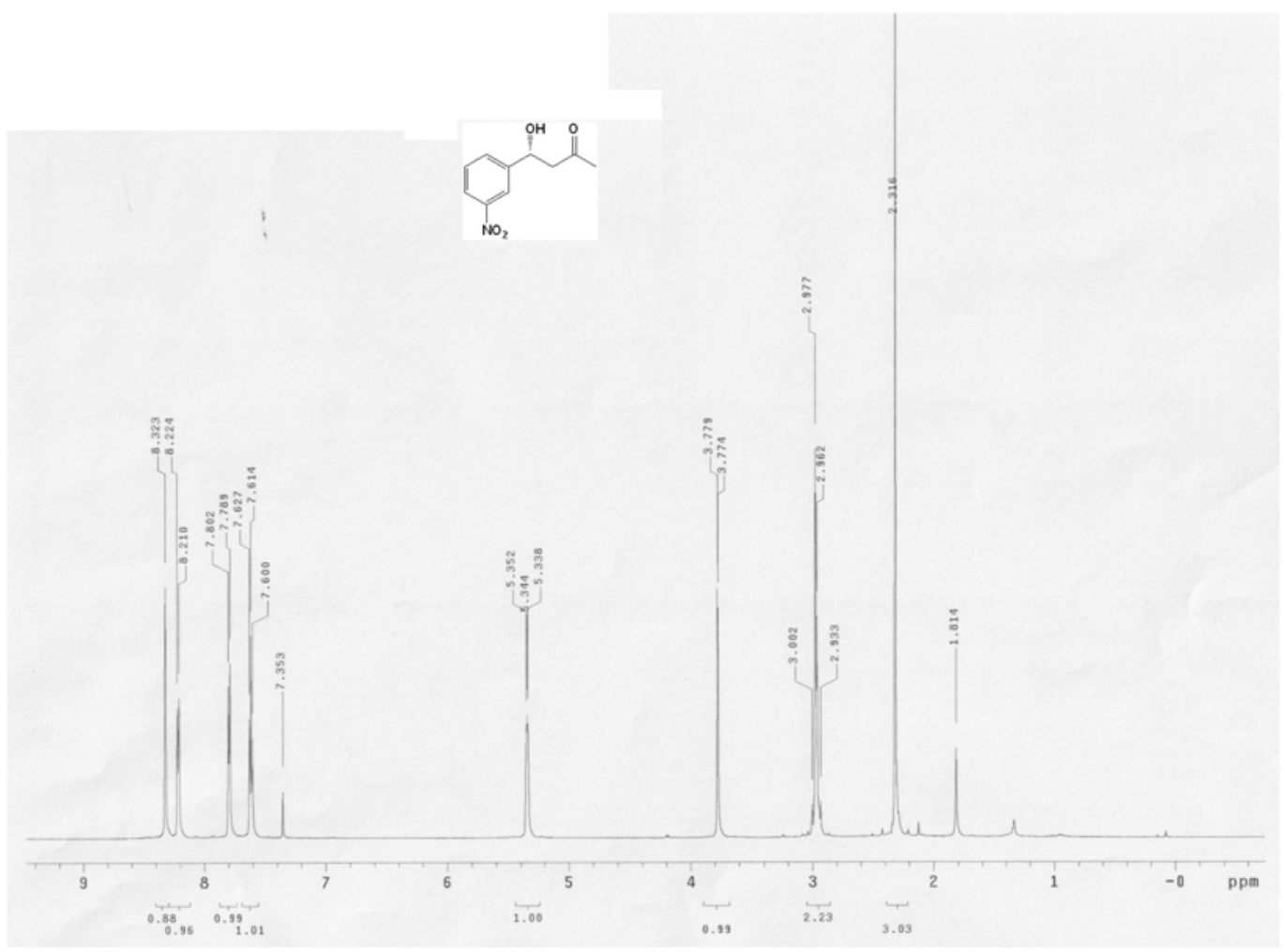

Figure S25. ${ }^{1} \mathrm{H}$ NMR (600 MHZ, $\mathrm{CDCl}_{3}$ ) spectrum of $\mathbf{5 c}$. 


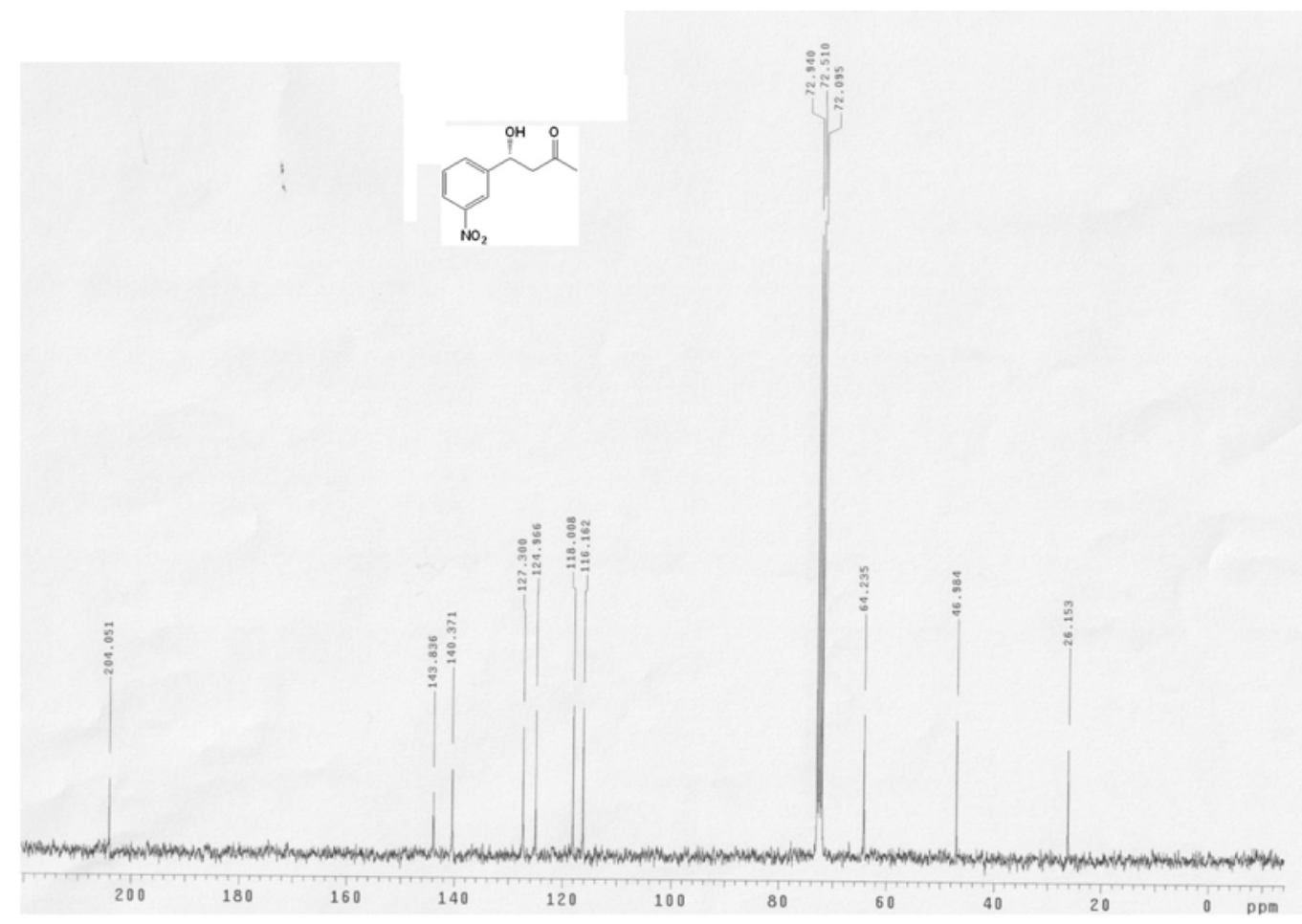

Figure S26. ${ }^{13} \mathrm{C}$ NMR $\left(75 \mathrm{MHZ}, \mathrm{CDCl}_{3}\right)$ spectrum of 5c.

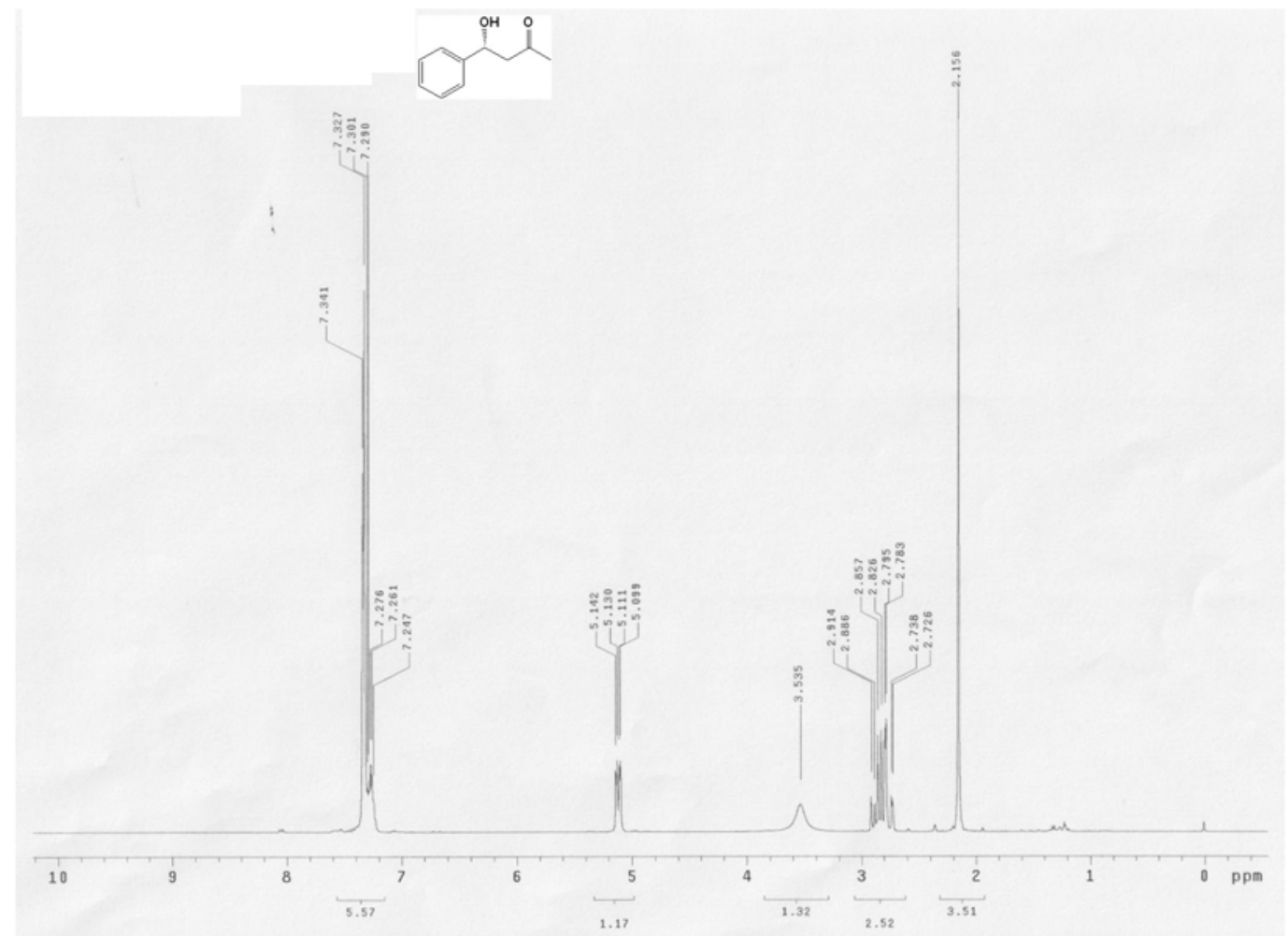

Figure S27. ${ }^{1} \mathrm{H}$ NMR (300 MHZ, $\mathrm{CDCl}_{3}$ ) spectrum of $\mathbf{5 d}$. 


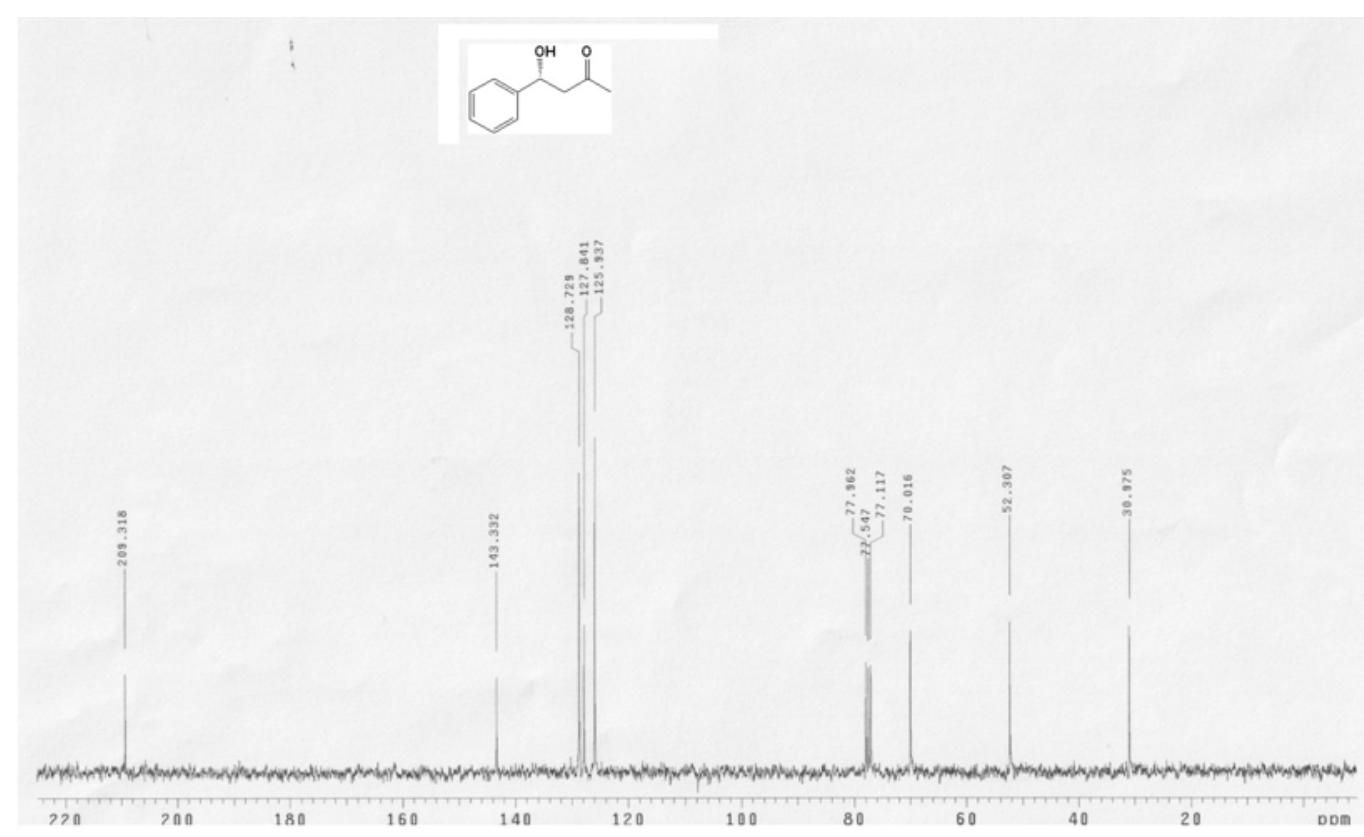

Figure S28. ${ }^{13} \mathrm{C}$ NMR (75 MHZ, $\mathrm{CDCl}_{3}$ ) spectrum of $\mathbf{5 d}$.

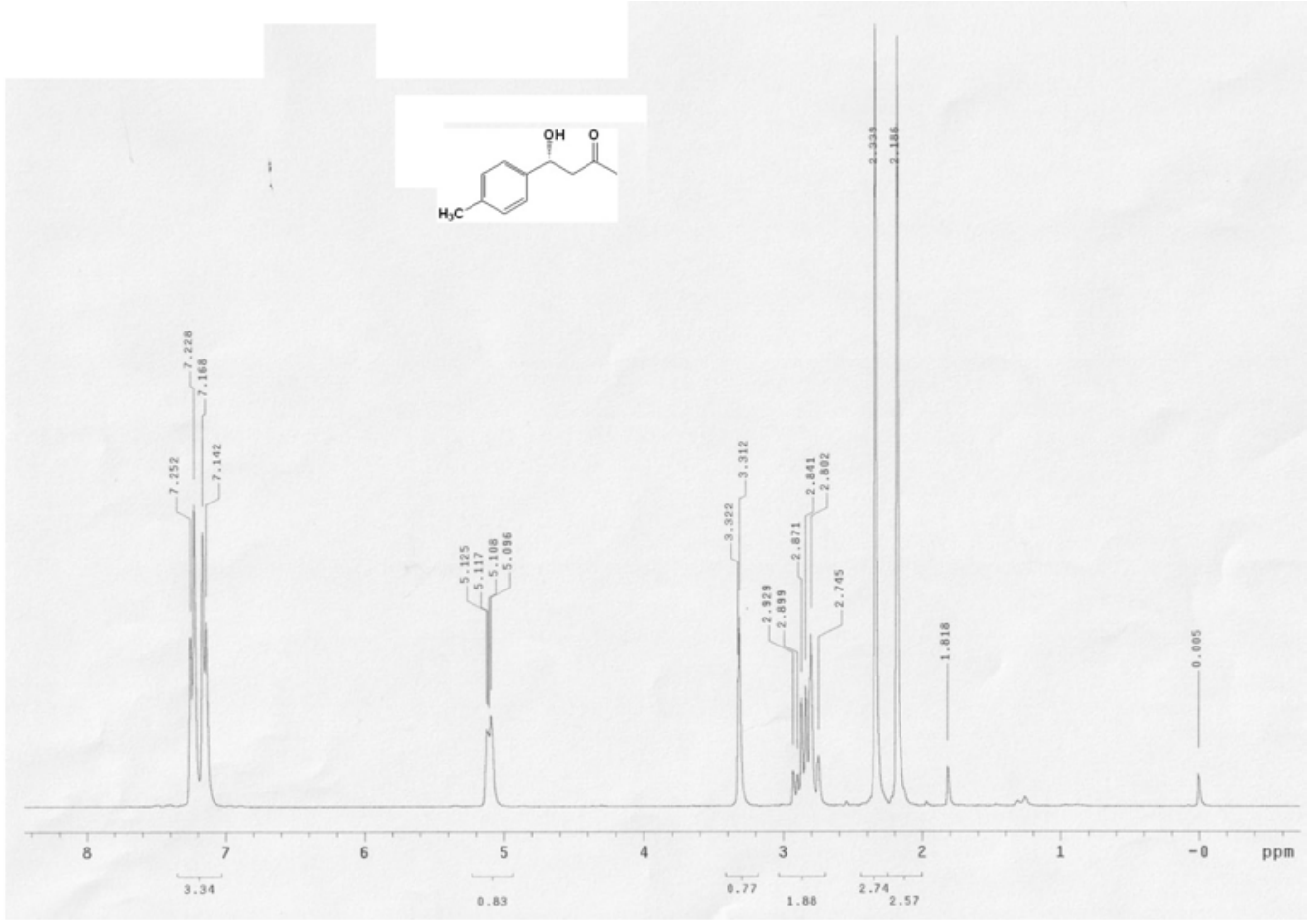

Figure S29. ${ }^{1} \mathrm{H}$ NMR (300 MHZ, $\mathrm{CDCl}_{3}$ ) spectrum of $5 \mathbf{e}$. 


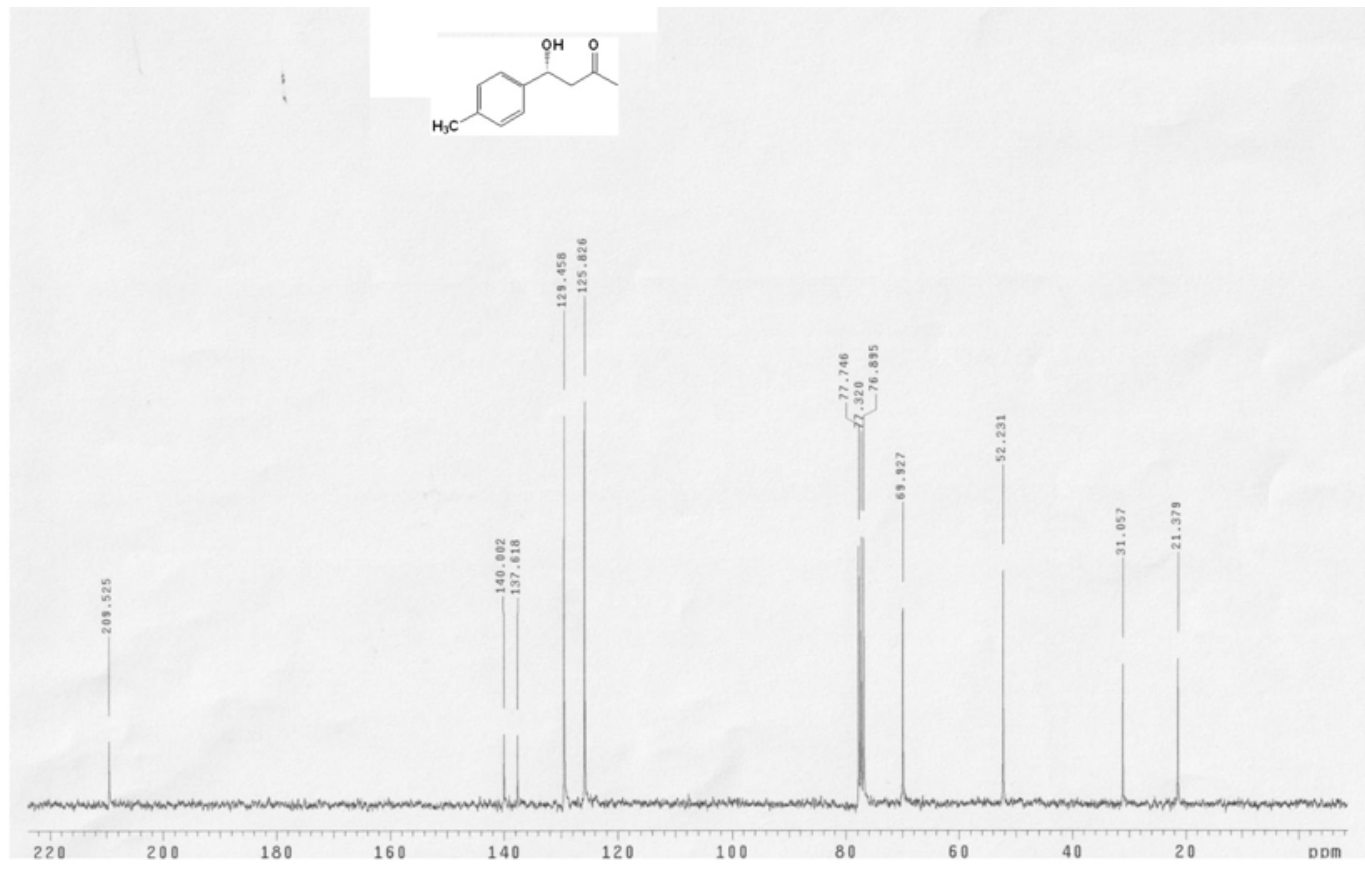

Figure S30. ${ }^{13} \mathrm{C}$ NMR (75 MHZ, $\left.\mathrm{CDCl}_{3}\right)$ spectrum of $\mathbf{5 e}$.

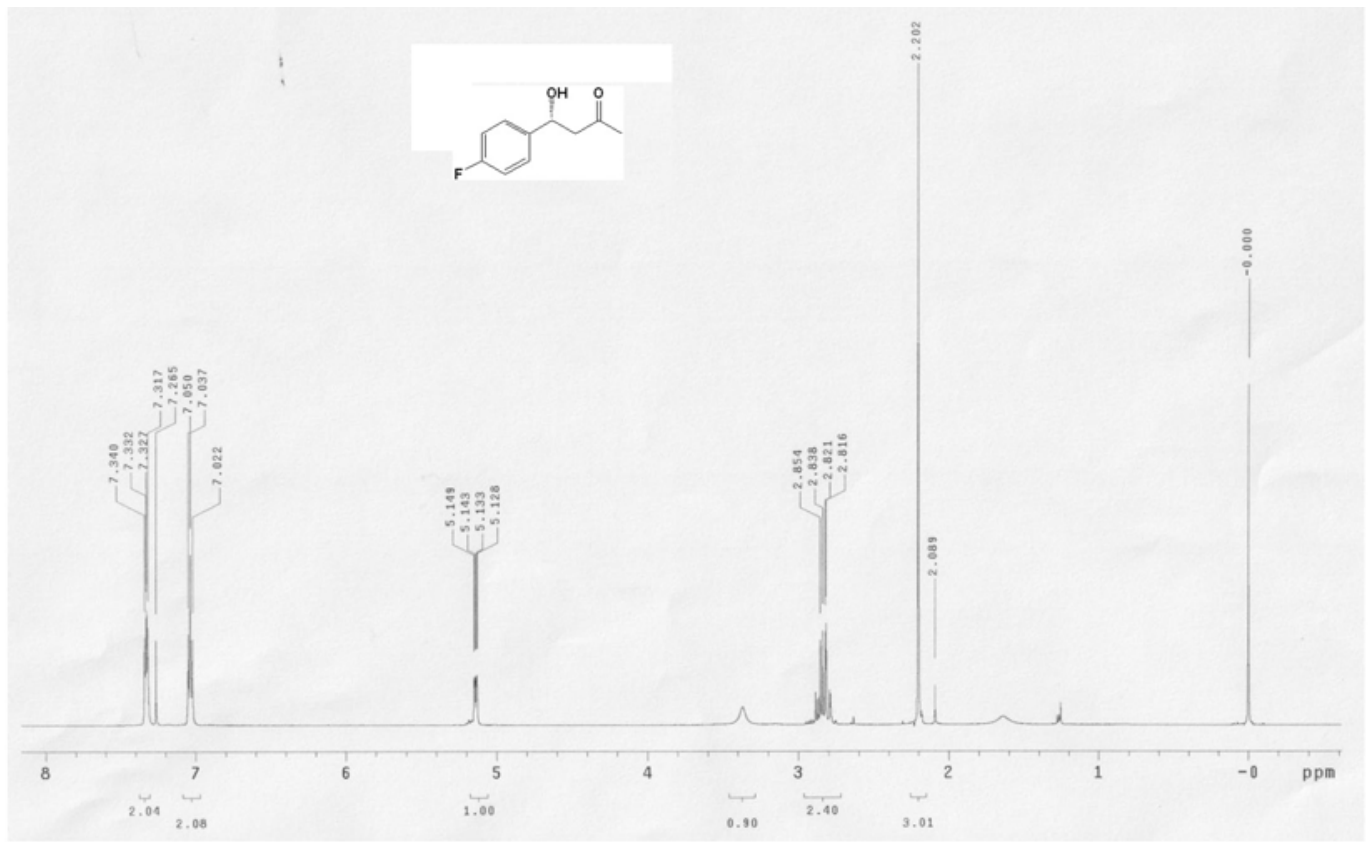

Figure S31. ${ }^{1} \mathrm{H}$ NMR (600 $\mathrm{MHZ}, \mathrm{CDCl}_{3}$ ) spectrum of $\mathbf{5 f}$. 


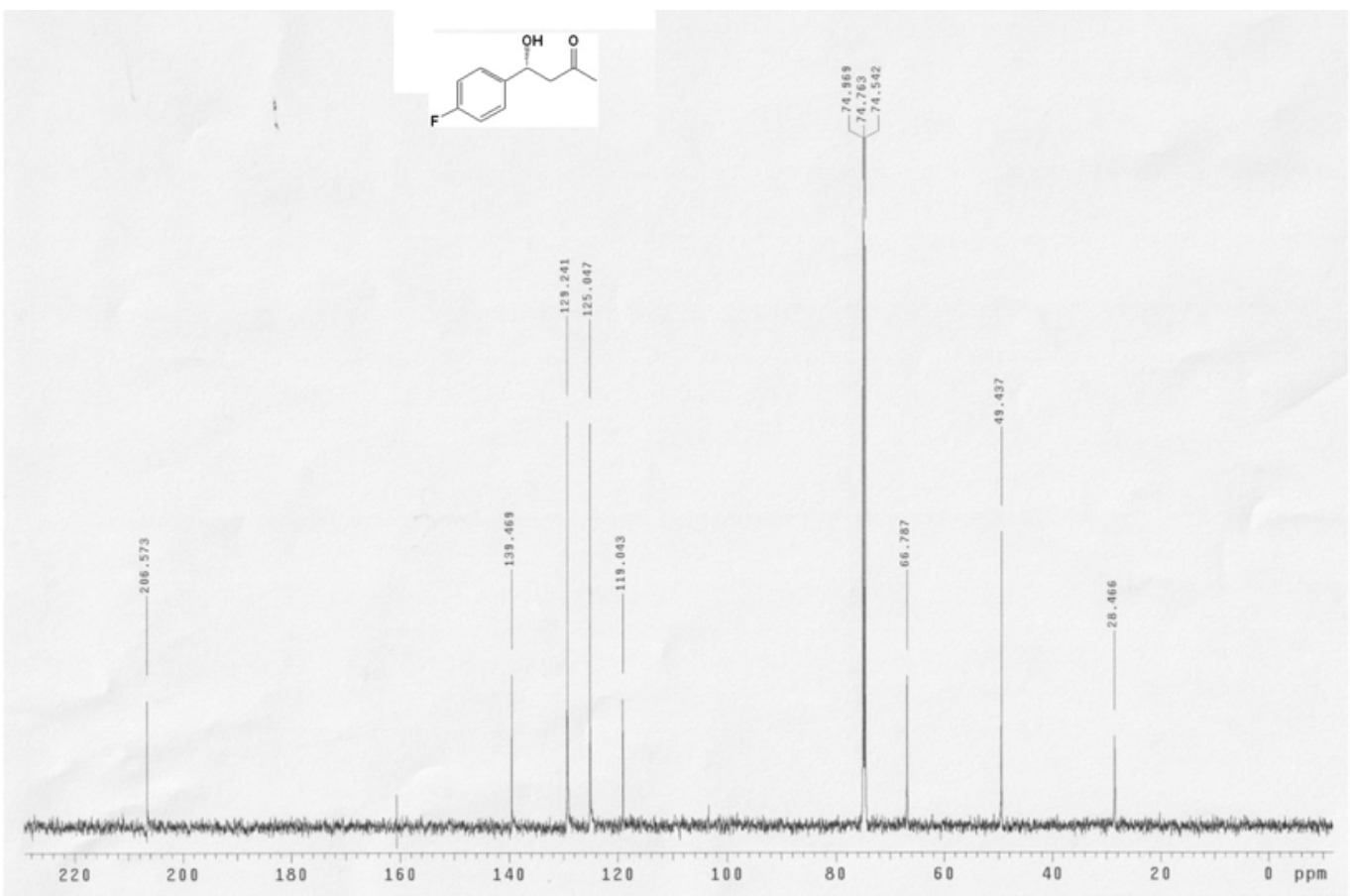

Figure S32. ${ }^{13} \mathrm{C}$ NMR (125 MHZ, $\mathrm{CDCl}_{3}$ ) spectrum of $\mathbf{5 f}$.

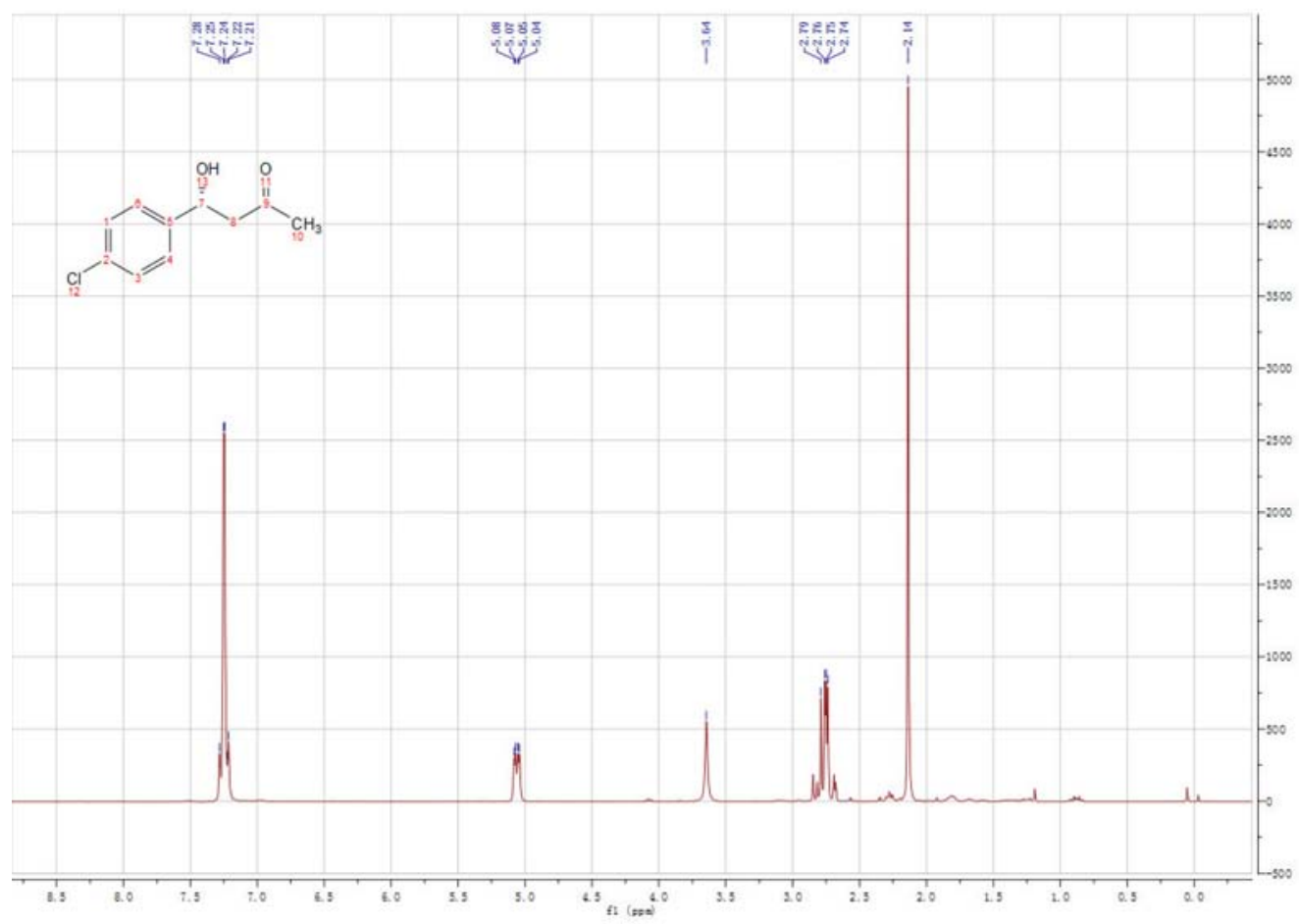

Figure S33. ${ }^{1} \mathrm{H}$ NMR (300 MHZ, $\mathrm{CDCl}_{3}$ ) spectrum of $\mathbf{5 g}$. 
Vol. 22, No. 9, 2011

Chang et al.

S19

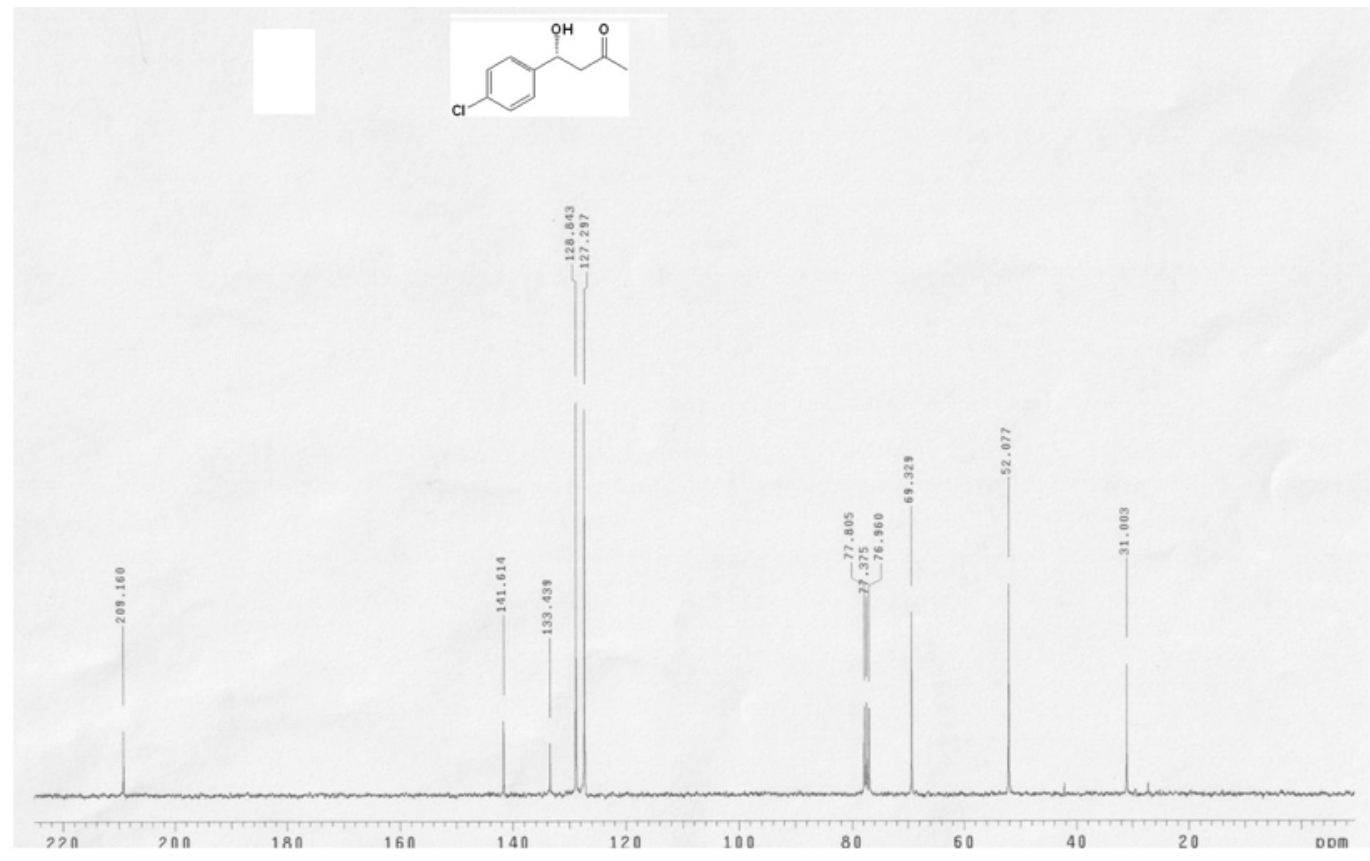

Figure S34. ${ }^{13} \mathrm{C}$ NMR (75 MHZ, $\mathrm{CDCl}_{3}$ ) spectrum of $\mathbf{5 g}$.
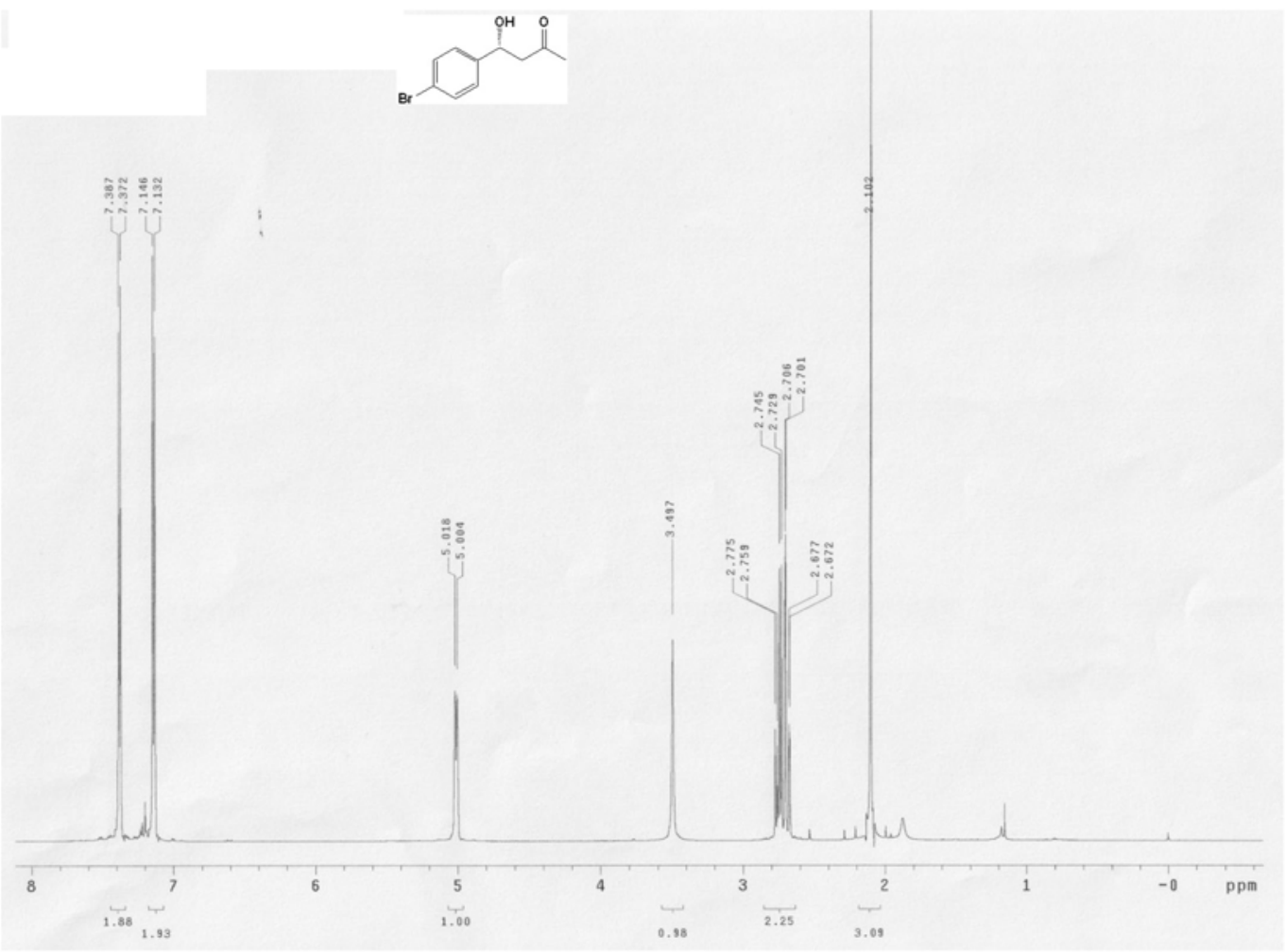

Figure S35. ' $\mathrm{H}$ NMR (600 MHZ, $\left.\mathrm{CDCl}_{3}\right)$ spectrum of $\mathbf{5 h}$. 


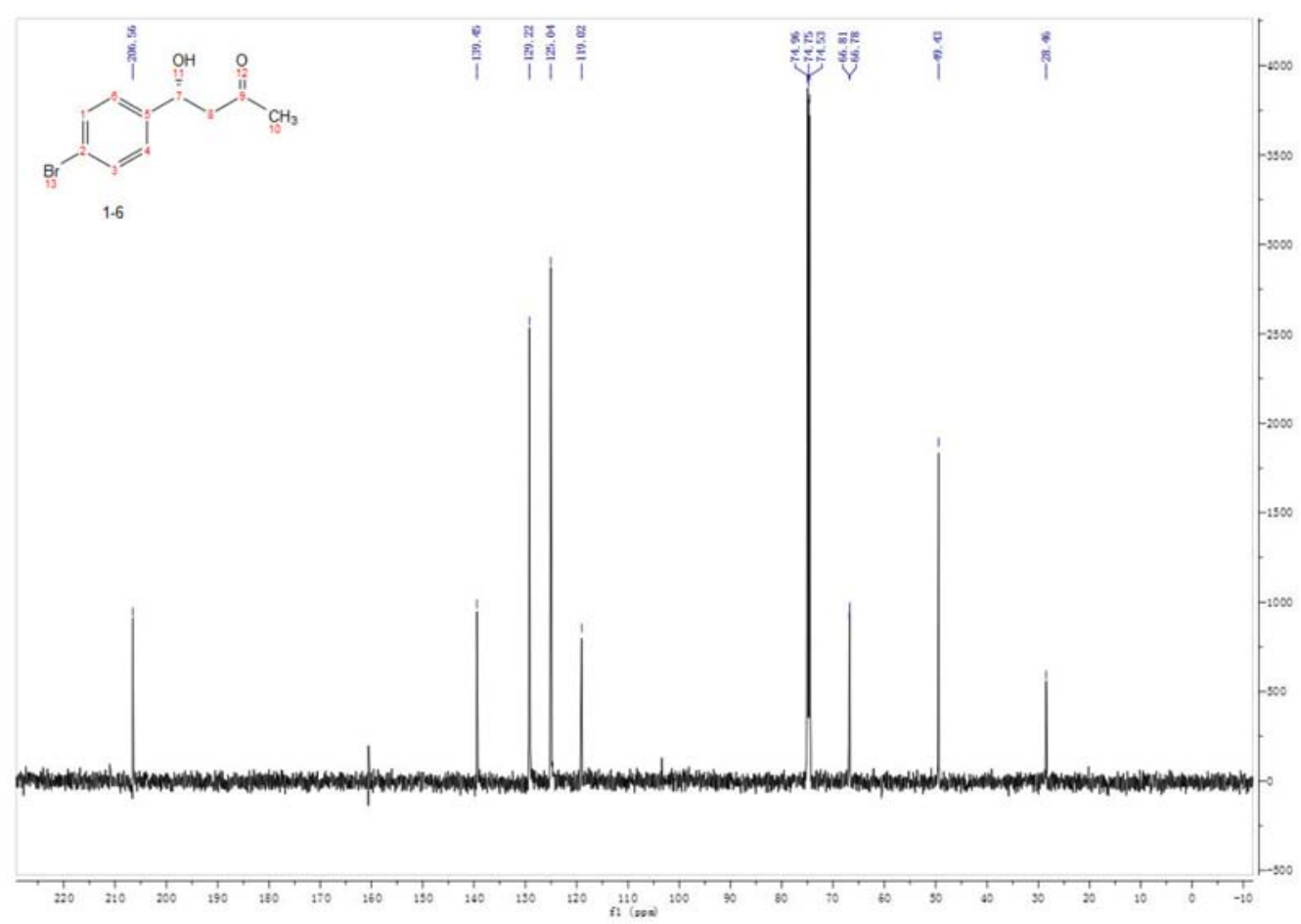

Figure S36. ${ }^{13} \mathrm{C}$ NMR (125 MHZ, $\mathrm{CDCl}_{3}$ ) spectrum of $\mathbf{5 h}$.

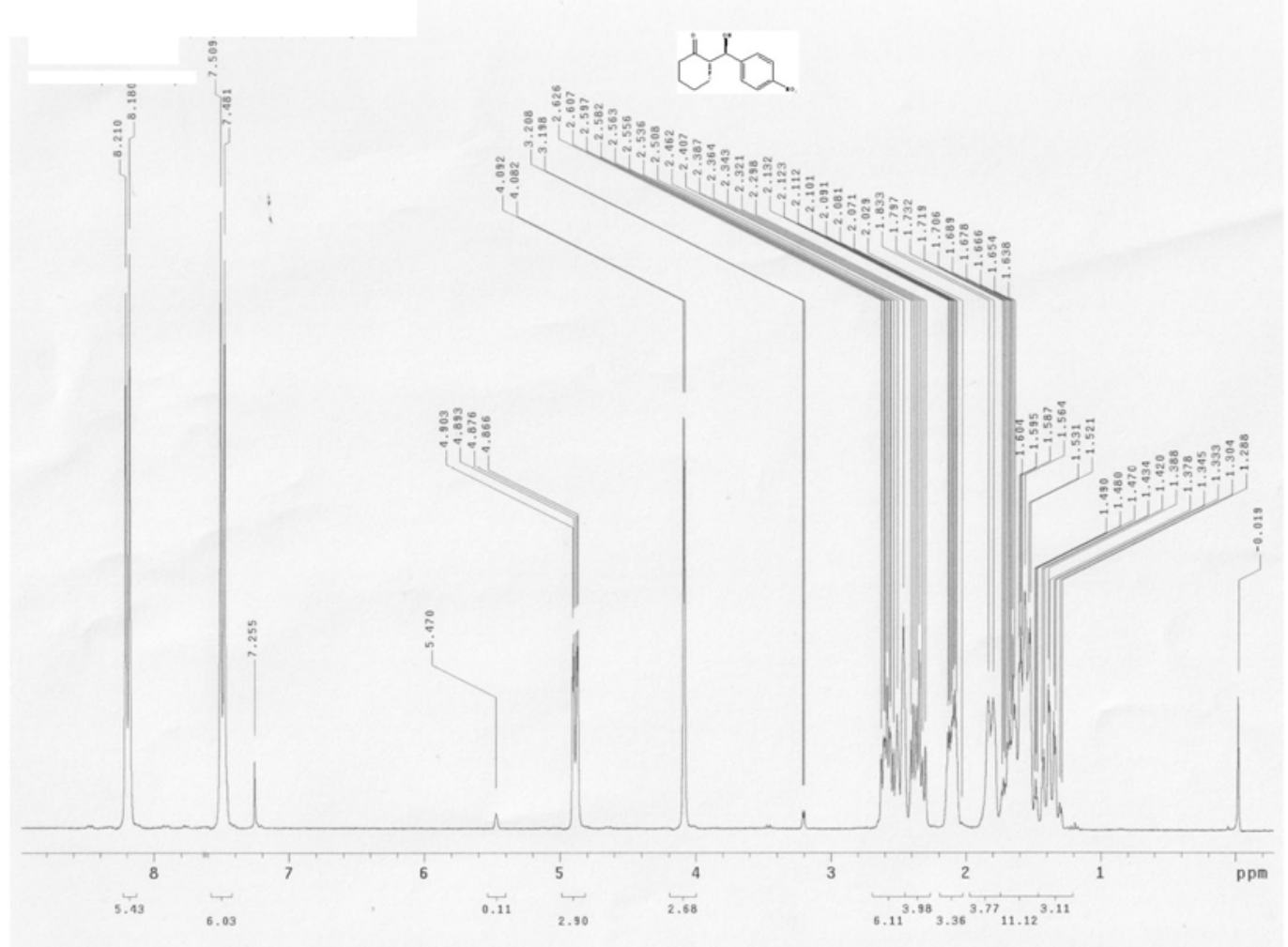

Figure S37. ${ }^{1} \mathrm{H}$ NMR (300 MHZ, $\mathrm{CDCl}_{3}$ ) spectrum of $\mathbf{7}$. 


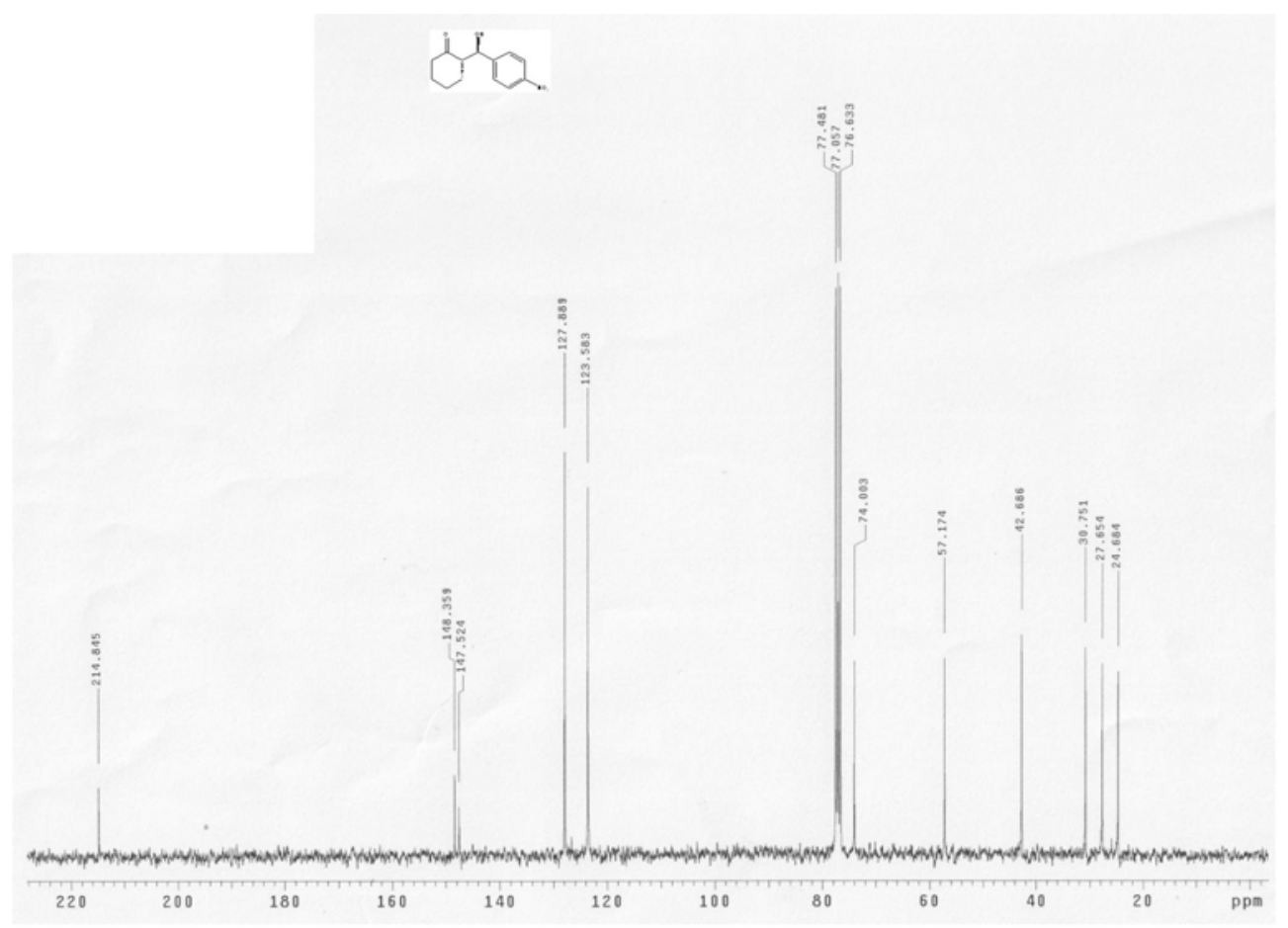

Figure S38. ${ }^{13} \mathrm{C}$ NMR (75 MHZ, $\mathrm{CDCl}_{3}$ ) spectrum of 7.

HPLC conditions for the aldol products

4-Hydroxy-4-(4-nitrophenyl) butan-2-one (5a)<smiles>CC(=O)C[C@H](O)c1ccc([N+](=O)[O-])cc1</smiles>

The optical purity was determined by HPLC on chiralpak AS-H column [hexane:2-propanol, 70:30]; flow rate $1.0 \mathrm{~mL} \mathrm{~min}^{-1} ; \lambda=210 \mathrm{~nm}$; major: $\mathrm{t}_{\mathrm{R}}=12.8 \mathrm{~min}$ and minor: $t_{R}=16.8$ min.

\section{4-Hydroxy-4-(2-nitrophenyl) butan-2-one (5b)}<smiles>CC(=O)C[C@H](O)c1ccccc1[N+](=O)[O-]</smiles>

The optical purity was determined by HPLC on chiralpak AS-H column [hexane:2-propanol, 70:30]; flow rate $1.0 \mathrm{~mL} \mathrm{~min}^{-1} ; \quad \lambda=220 \mathrm{~nm}$; minor: $\mathrm{t}_{\mathrm{R}}=8.3 \mathrm{~min}$ and major: $t_{R}=11.2 \mathrm{~min}$.
4-Hydroxy-4-(3-nitrophenyl) butan-2-one (5c)<smiles>CC(=O)C[C@@H](O)c1cccc([N+](=O)[O-])c1</smiles>

The optical purity was determined by HPLC on chiralpak OJ-H column [hexane:2-propanol, 70:30]; flow rate $1.0 \mathrm{~mL} \mathrm{~min}^{-1} ; \lambda=220 \mathrm{~nm}$; major: $\mathrm{t}_{\mathrm{R}}=10.7 \mathrm{~min}$ and minor: $\mathrm{t}_{\mathrm{R}}=12.4 \mathrm{~min}$.

4-Hydroxy-4-phenylbutan-2-one (5d)<smiles>CC(=O)C[C@@H](O)c1ccccc1</smiles>

The optical purity was determined by HPLC on chiralpak AD-H column [hexane:2-propanol, 95:5]; flow

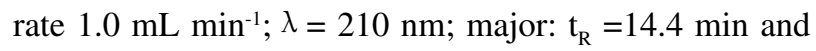
minor: $t_{R}=16.3 \mathrm{~min}$. 
(R)-4-Hydroxy-4-p-tolylbutan-2-one (5e)<smiles>CC(=O)C[C@H](O)c1ccc(C)cc1</smiles>

The optical purity was determined by HPLC on chiralpak AS-H column [hexane:2-propanol, 85:15]; flow rate $1.0 \mathrm{~mL} \mathrm{~min}{ }^{-1} ; \lambda=220 \mathrm{~nm}$; major: $\mathrm{t}_{\mathrm{R}}=9.8 \mathrm{~min}$ and minor: $\mathrm{t}_{\mathrm{R}}=12.2 \mathrm{~min}$.

4-(4-Fluorophenyl)-4-hydroxybutan-2-one ( $5 f)$<smiles>CC(=O)C[C@H](O)c1ccc(F)cc1</smiles>

The optical purity was determined by HPLC on chiralpak AS-H column [hexane:2-propanol, 70:3]; flow

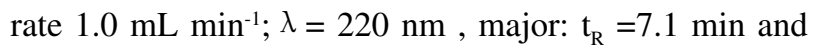
minor: $7.6 \mathrm{~min}$.

4-(4-Chlorophenyl)-4-hydroxybutan-2-one (5g)<smiles>CC(=O)C[C@@H](O)c1ccc(Cl)cc1</smiles>

The optical purity was determined by HPLC on chiralpak AS-H column [hexane:2-propanol, 80:20]; flow

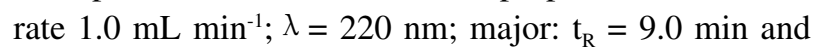
minor: $t_{R}=10.9 \min$.
4-(4-Bromophenyl)-4-hydroxybutan-2-one (5h)<smiles>CC(=O)C[C@H](O)c1ccc(Br)cc1</smiles>

The optical purity was determined by HPLC on chiralpak AS-H column [hexane:2-propanol, 70:30]; flow

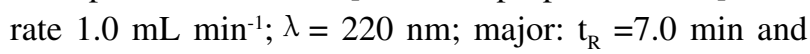
minor: $t_{R}=8.1 \mathrm{~min}$.

(S)-2-((R)-Hydroxy(4-nitrophenyl)methyl)cyclohexanone (7)<smiles>O=C1CCCC[C@H]1[C@H](O)c1ccc([N+](=O)[O-])cc1</smiles>

The optical purity was determined by HPLC on chiralpak AD-H column [hexane:2-propanol, 80:20]; flow rate $0.5 \mathrm{~mL} \mathrm{~min}^{-1} ; \lambda=220 \mathrm{~nm}$; mimor: $\mathrm{t}_{\mathrm{R}}=21.6 \mathrm{~min}$ and 22.7, major: $\mathrm{t}_{\mathrm{R}}=24.6$ and $31.4 \mathrm{~min}$. 


\section{HPLC spectra}

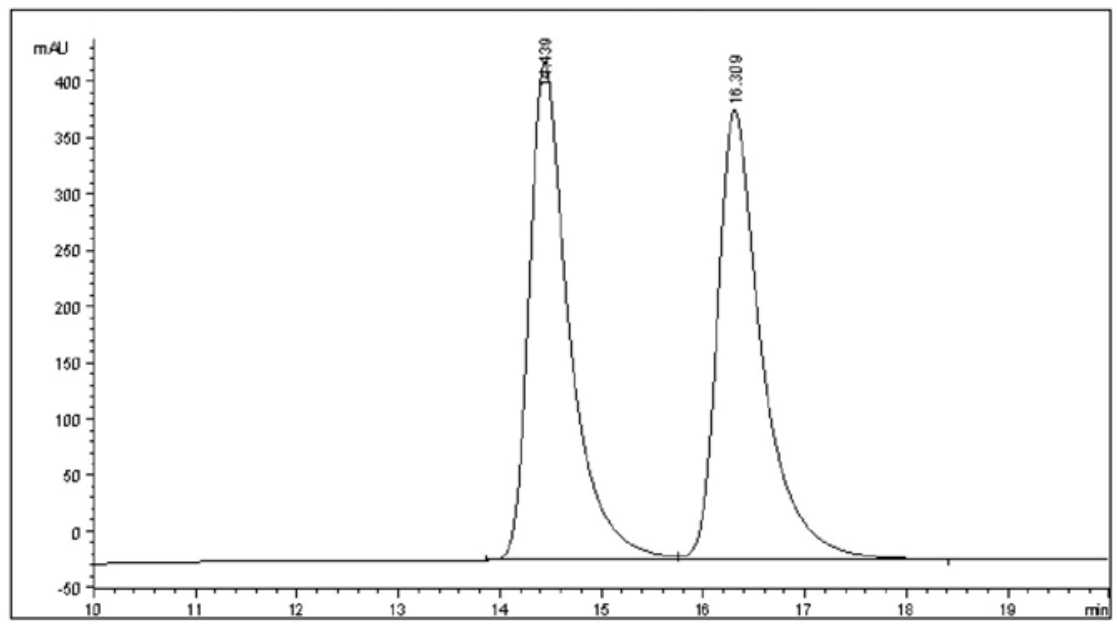

Figure S39. HPLC spectrum of $\mathbf{5 a}$ (racemic).

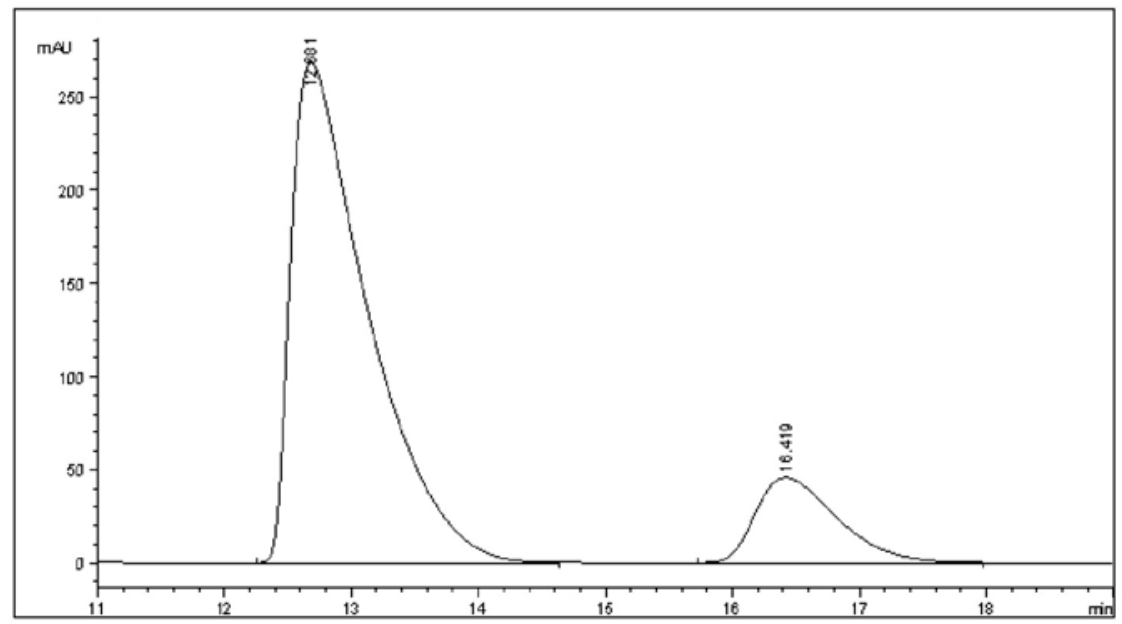

Figure S40. HPLC spectrum of $\mathbf{5 a}$ (Table 1, entry 5).

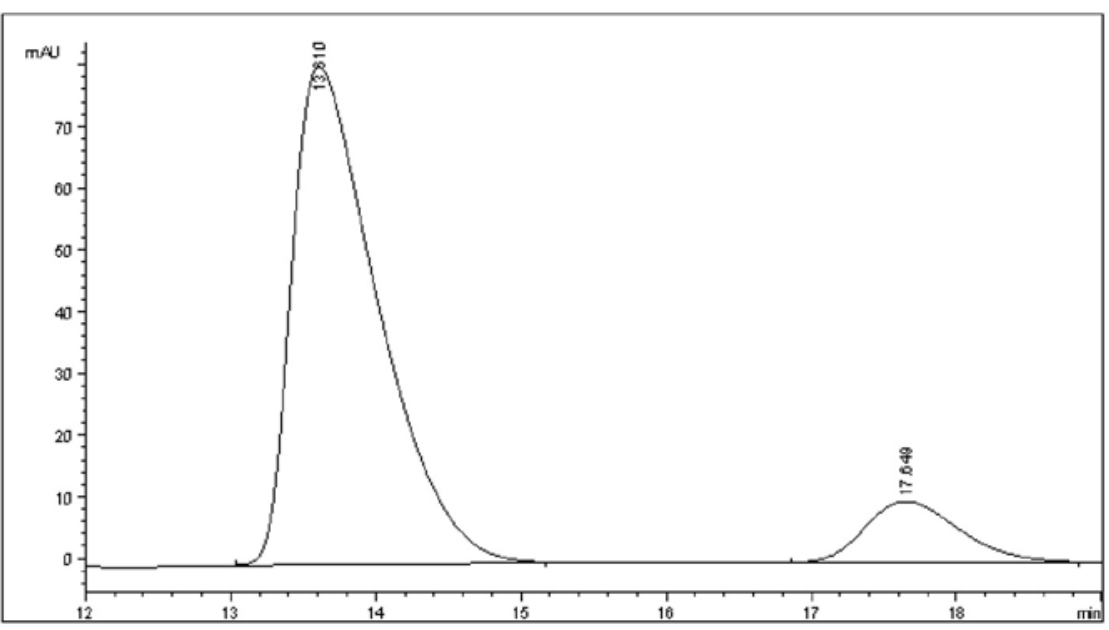

Figure S41. HPLC spectrum of $\mathbf{5 a}$ (Table 1, entry 6). 


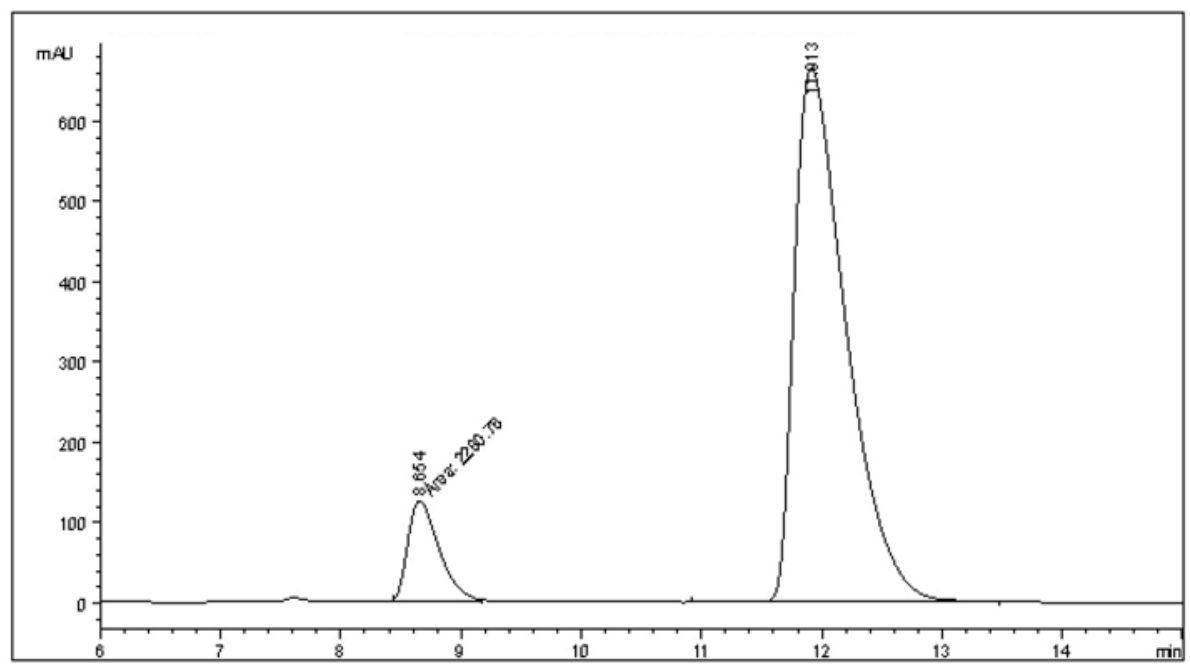

Figure S42. HPLC spectrum of $\mathbf{5 b}$ (Table 1, entry 9).

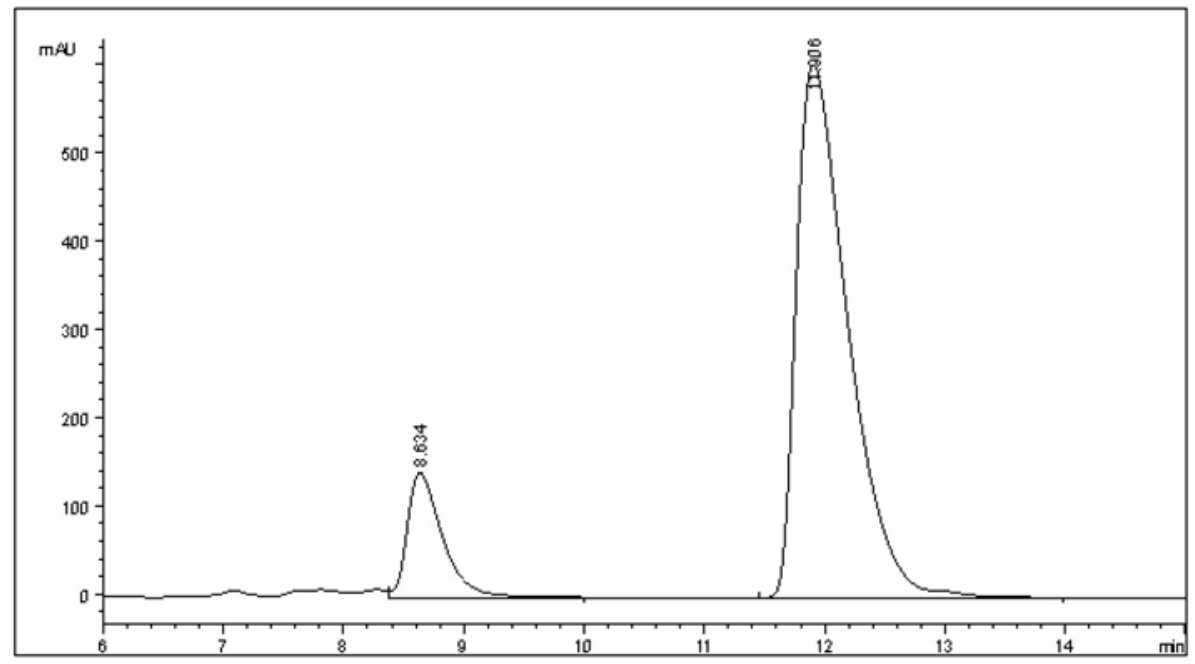

Figure S43. HPLC spectrum of $\mathbf{5 b}$ (Table 1, entry 10).

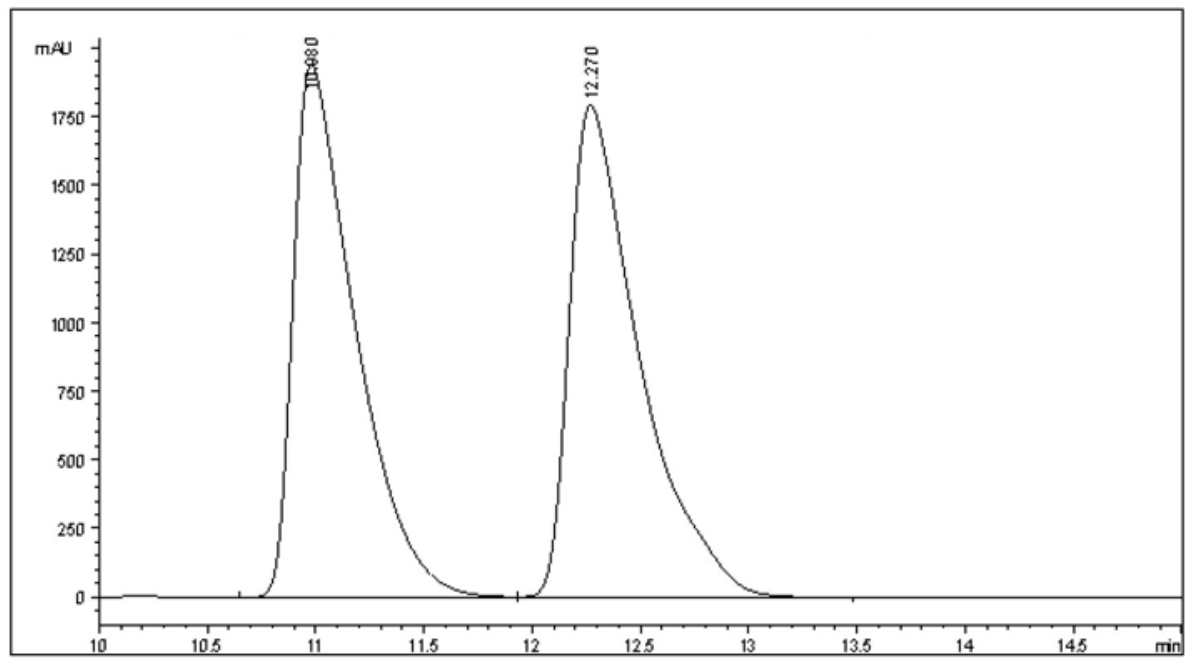

Figure S44. HPLC spectrum of $\mathbf{5 c}$ (racemic). 


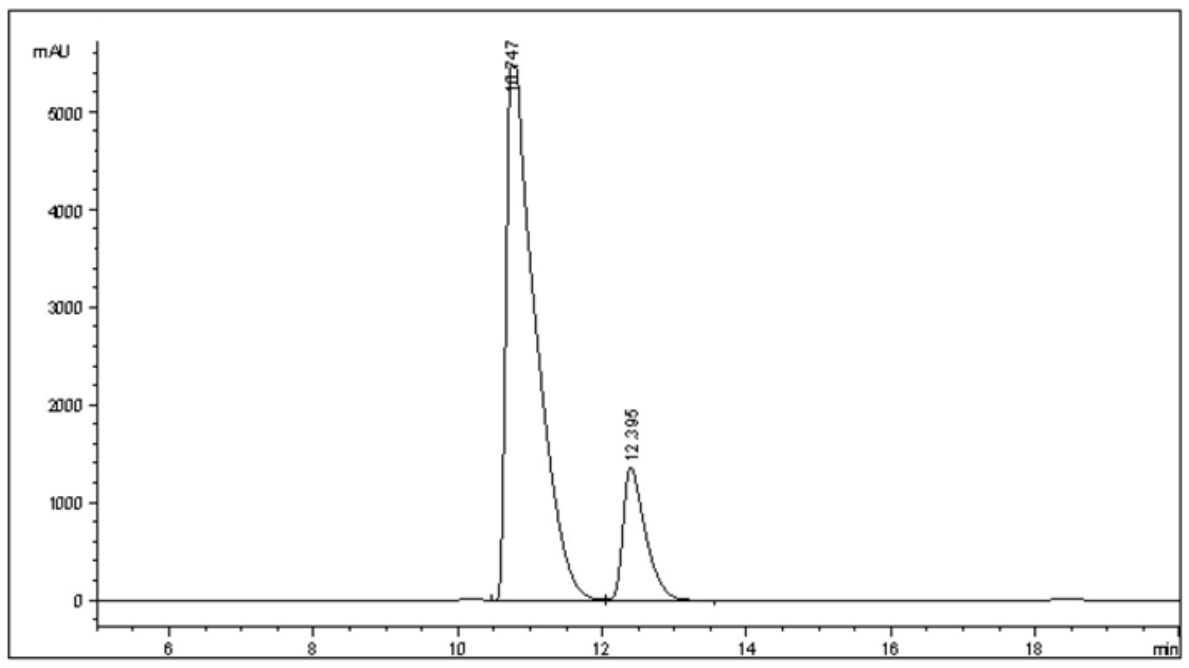

Figure S45. HPLC spectrum of 5c (Table 1, entry 14).

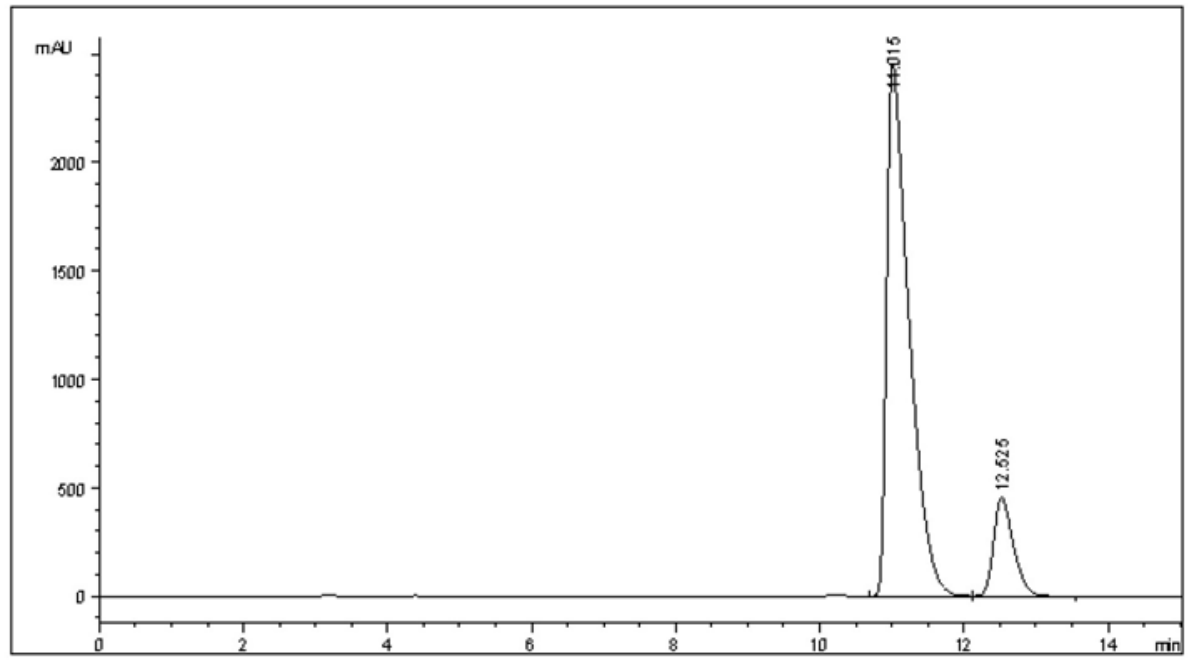

Figure S46. HPLC spectrum of 5c (Table 1, entry 15).

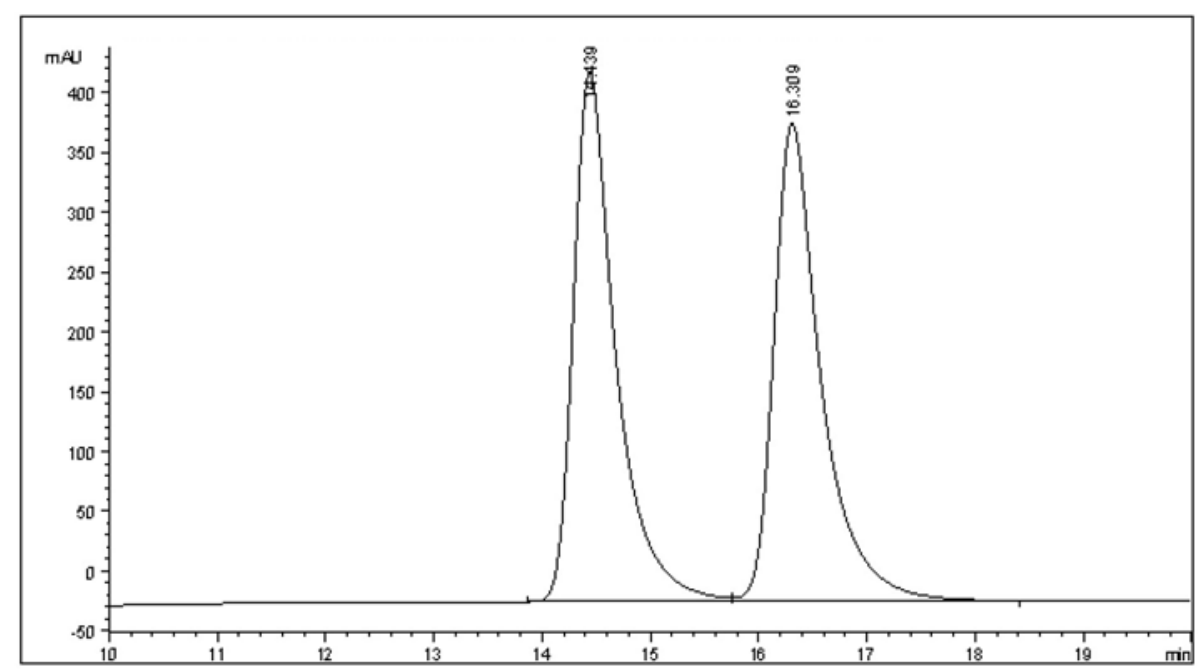

Figure S47. HPLC spectrum of $\mathbf{5 d}$ (racemic). 


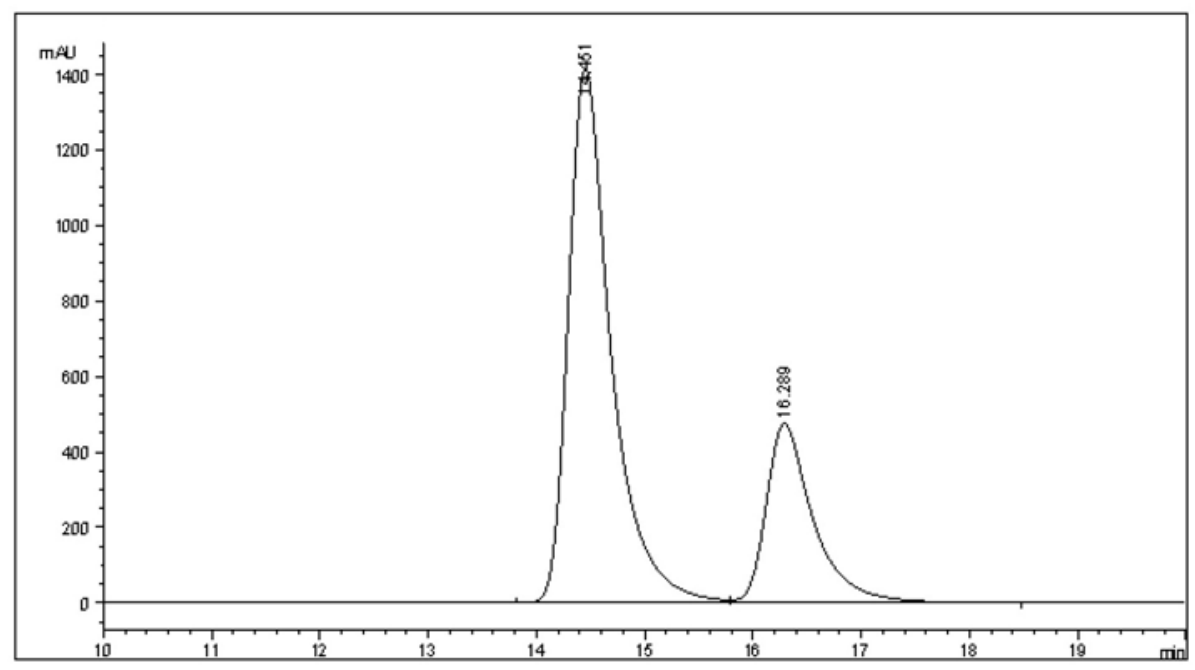

Figure S48. HPLC spectrum of 5d (Table 1, entry 16).

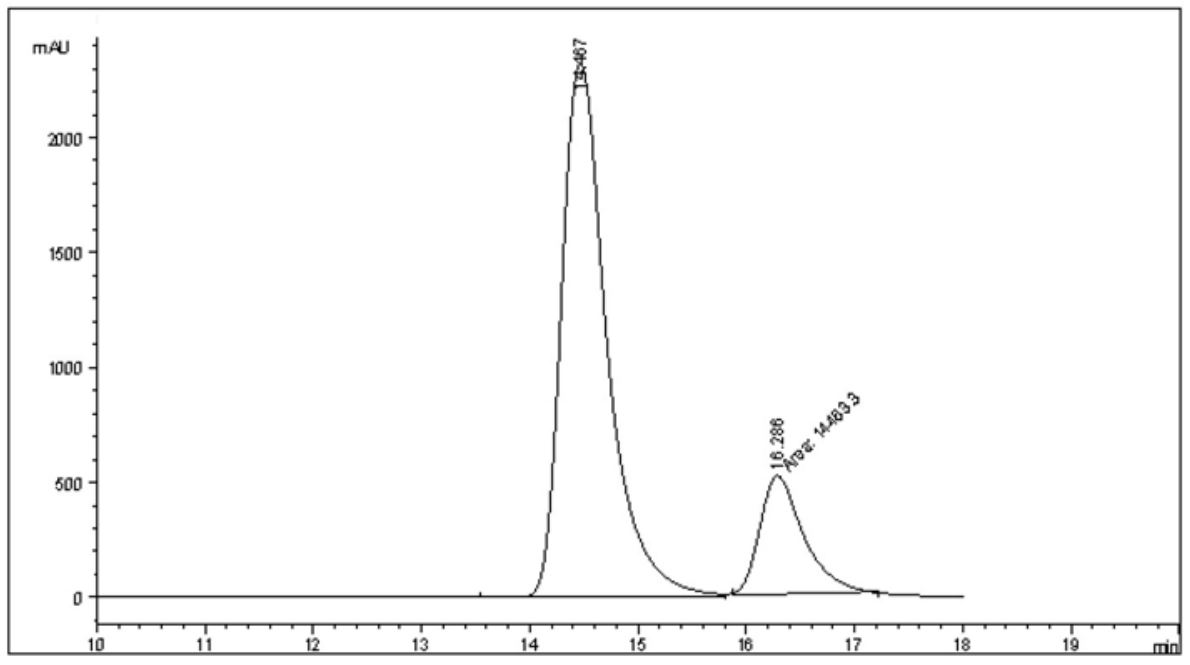

Figure S49. HPLC spectrum of 5d (Table 1, entry 17).

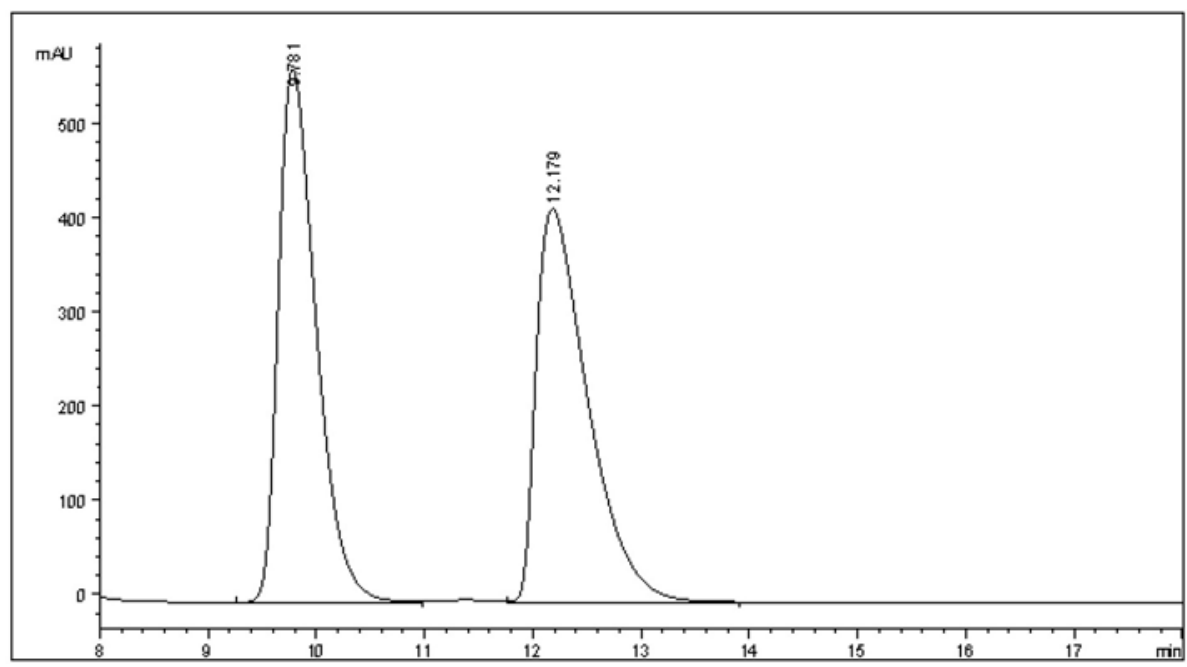

Figure S50. HPLC spectrum of $\mathbf{5 e}$ (racemic). 


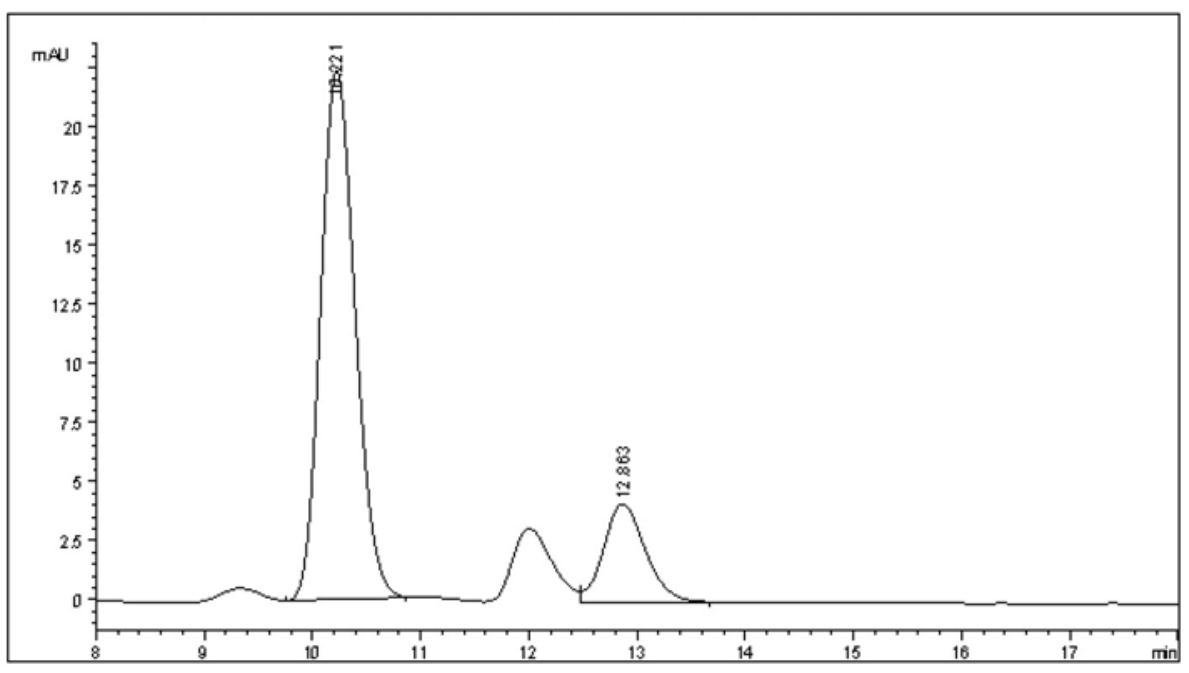

Figure S51. HPLC spectrum of 5e (Table 1, entry 29).

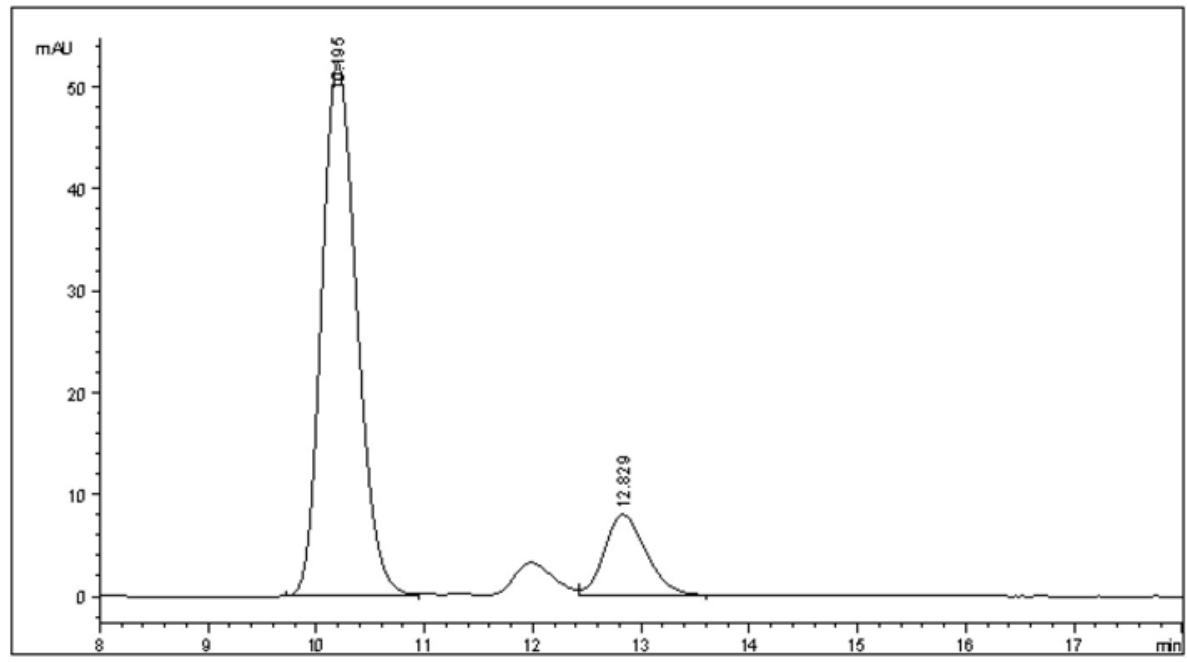

Figure S52. HPLC spectrum of 5e (Table 1, entry 30).

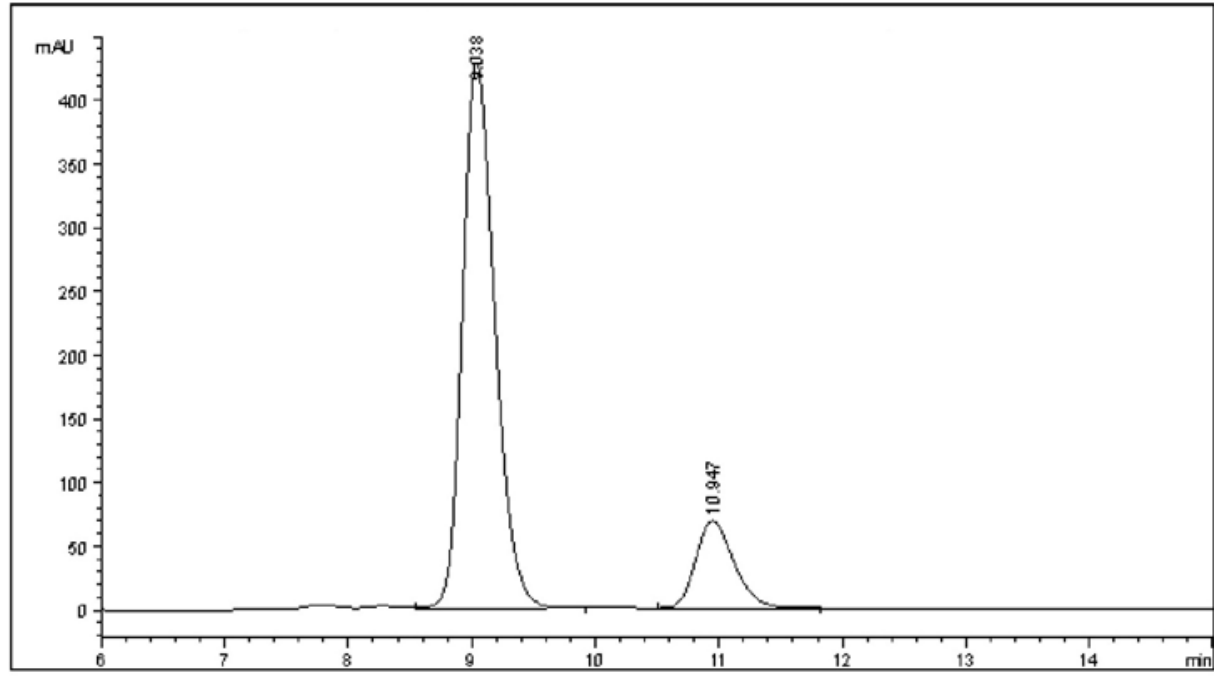

Figure S53. HPLC spectrum of $\mathbf{5 f}$ (Table 1, entry 20). 


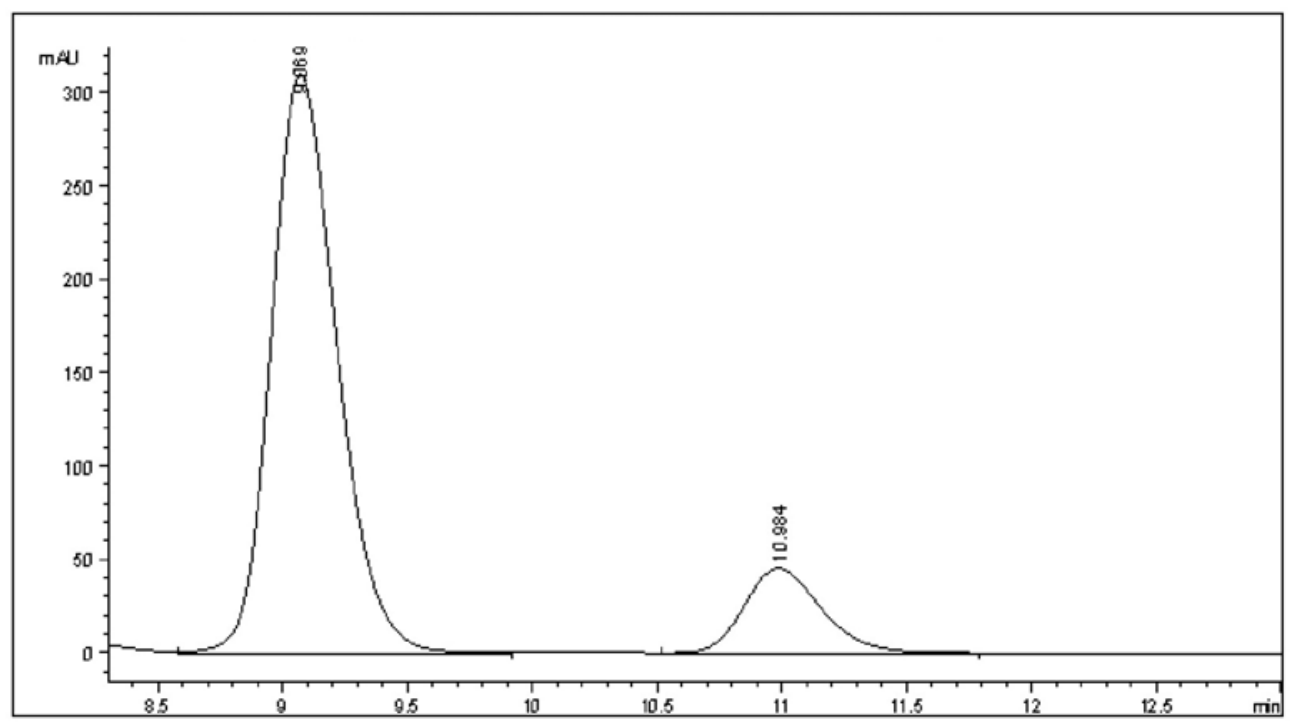

Figure S54. HPLC spectrum of $\mathbf{5 f}$ (Table 1, entry 21).

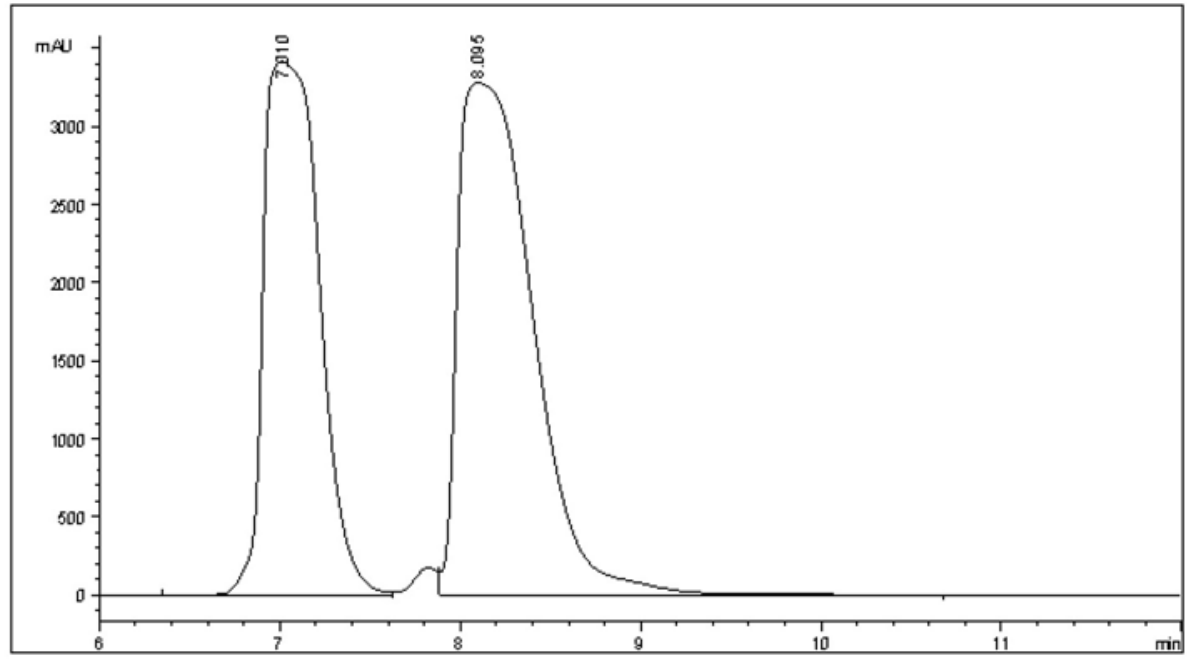

Figure S55. HPLC spectrum of $\mathbf{5 g}$ (racemic).

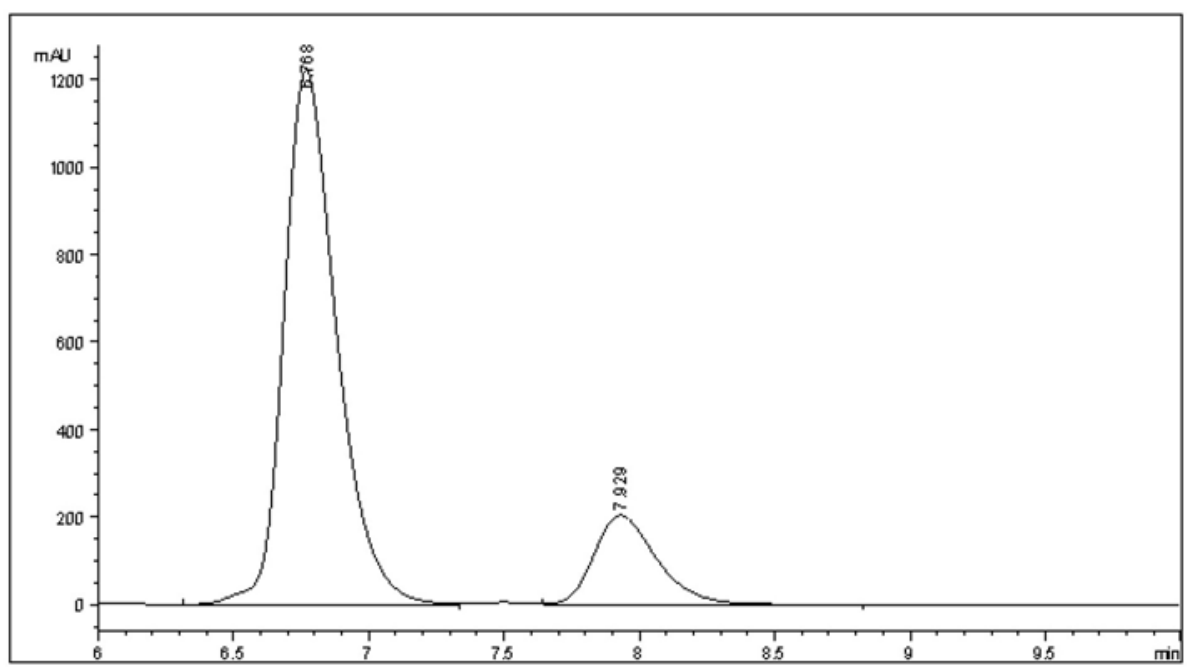

Figure S56. HPLC spectrum of $\mathbf{5 g}$ (Table 1, entry 27). 


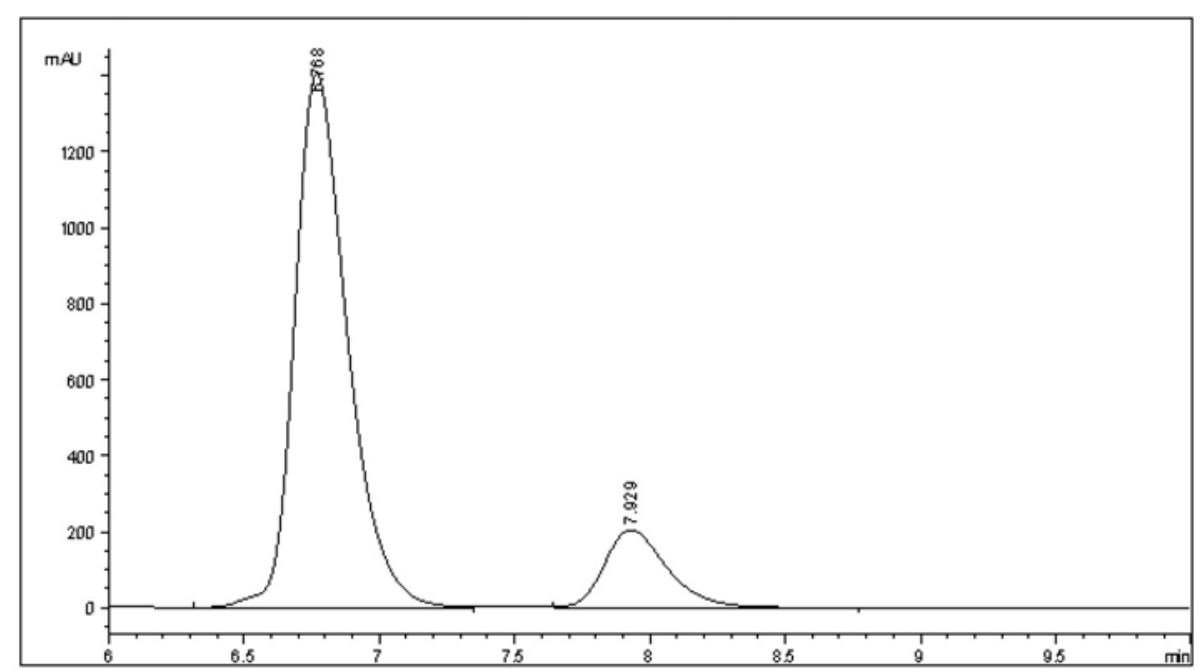

Figure S57. HPLC spectrum of 5g (Table 1, entry 28).

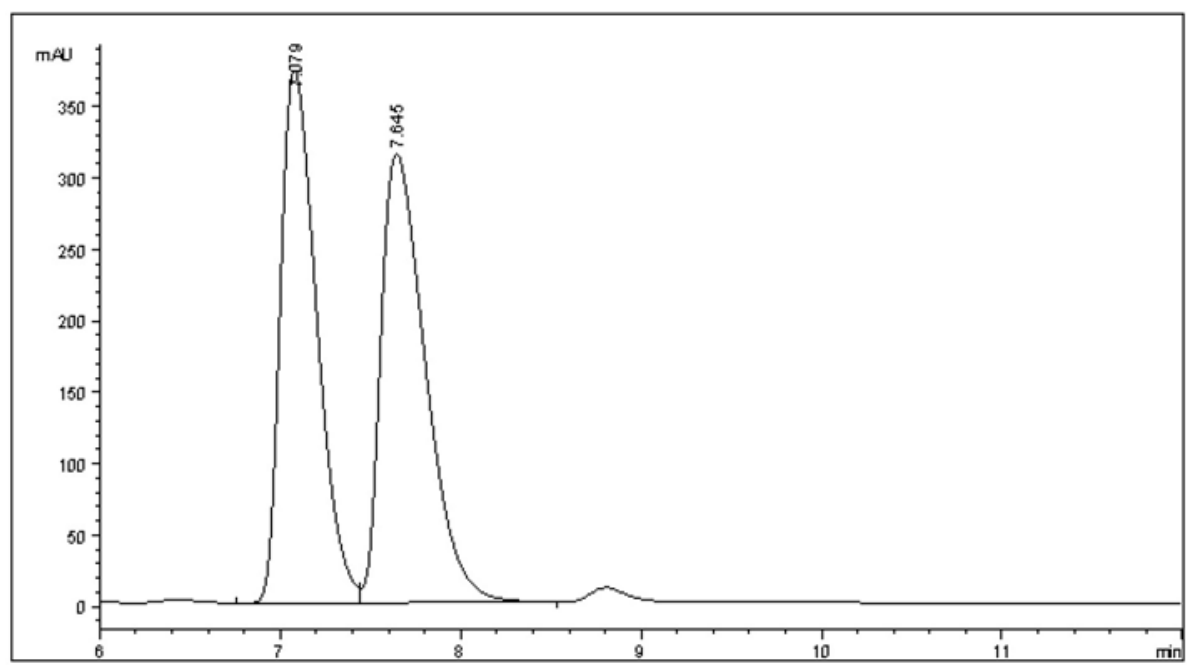

Figure S58. HPLC spectrum of $\mathbf{5 h}$ (racemic).

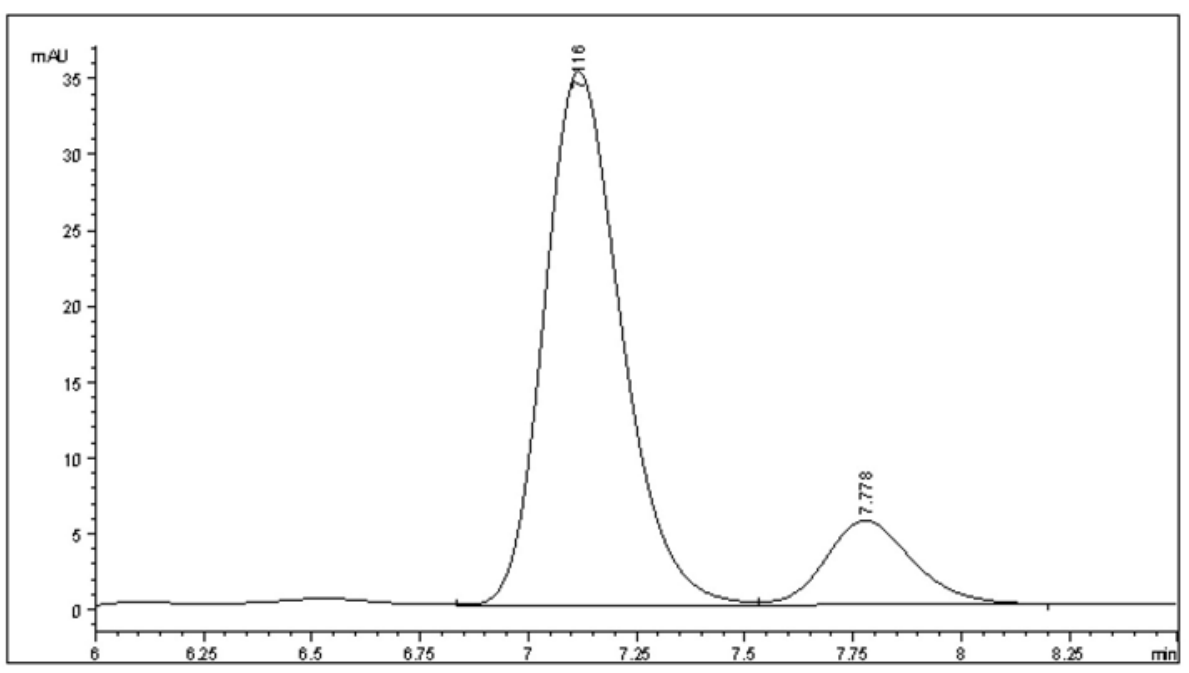

Figure S59. HPLC spectrum of $\mathbf{5 h}$ (Table 1, entry 29). 


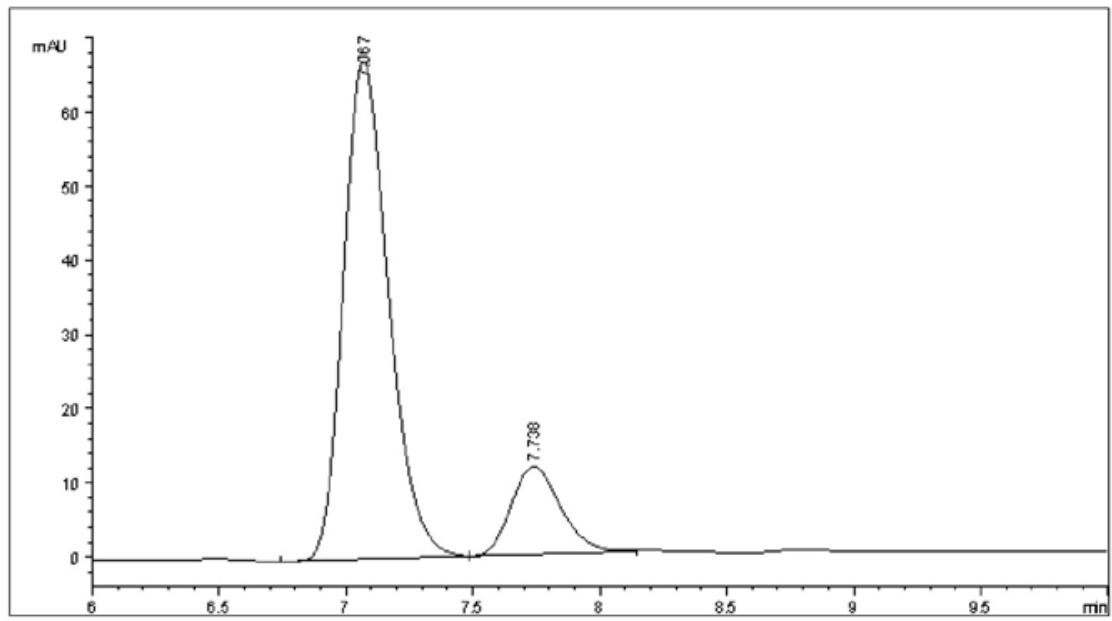

Figure S60. HPLC spectrum of $\mathbf{5 h}$ (Table 1, entry 30).

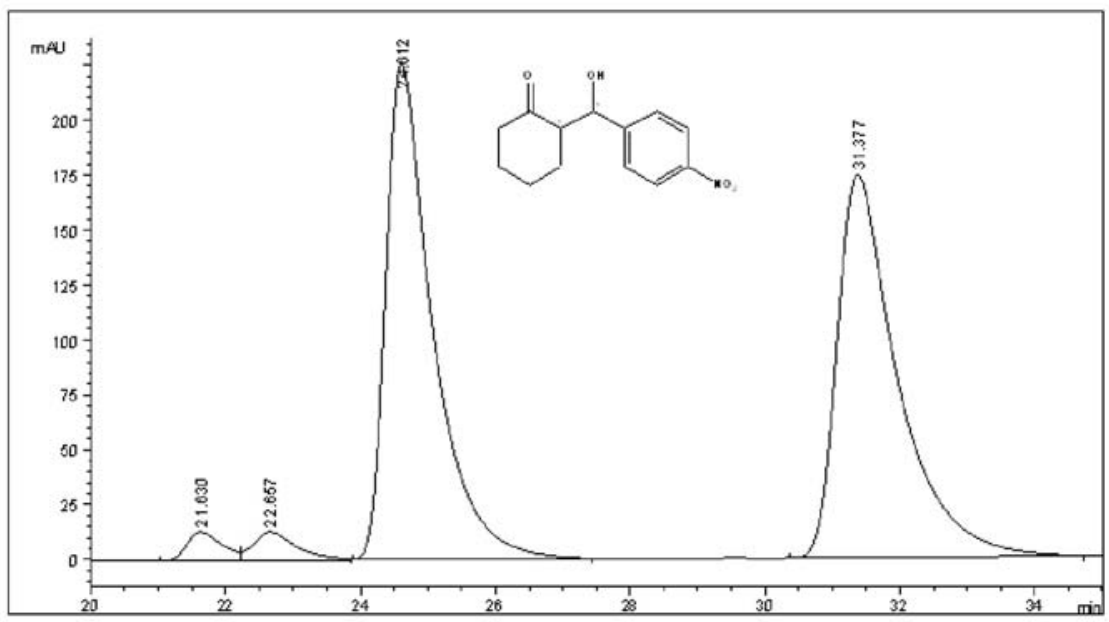

Figure S61. HPLC spectrum of 7 (racemic).

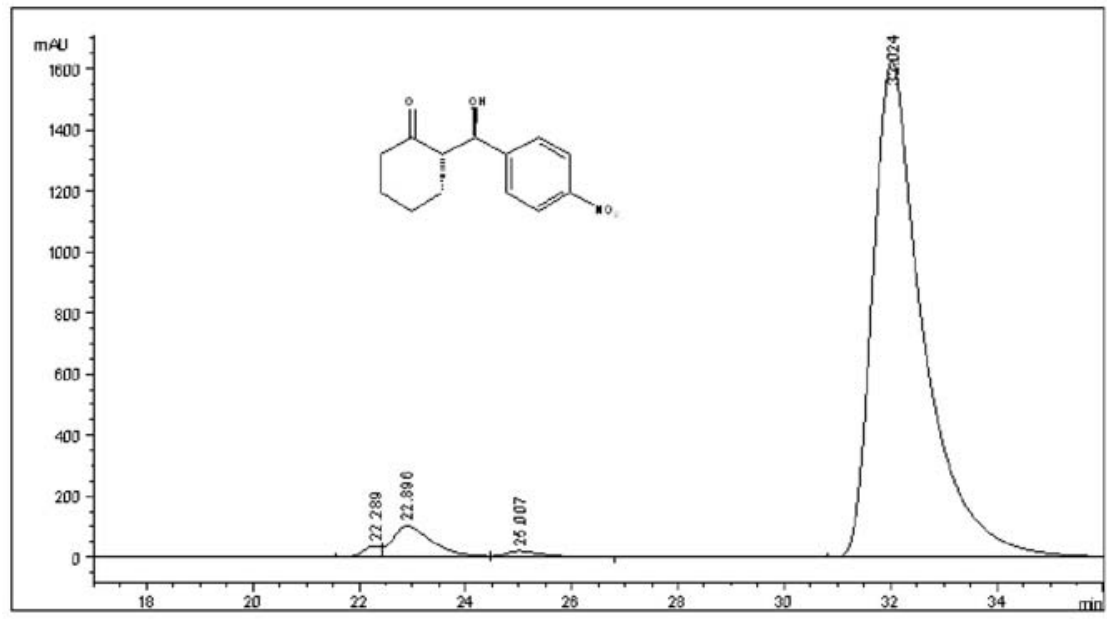

Figure S62. HPLC spectrum of 7 (Table 2, entry 5).

\section{References}

1. Zhou Y.; Shan Z.; Tetrahedron: Asymmetry 2006, 17, 1671.

2. Jia Y. N.; Wu, F. C.; Ma, X.; Zhu, G. J.; Da, C. S.; Tetrahedron Lett. 2009, 50, 3059. 